\title{
The Art of Catching and Probing Single Molecules
}

Avijit Kumar

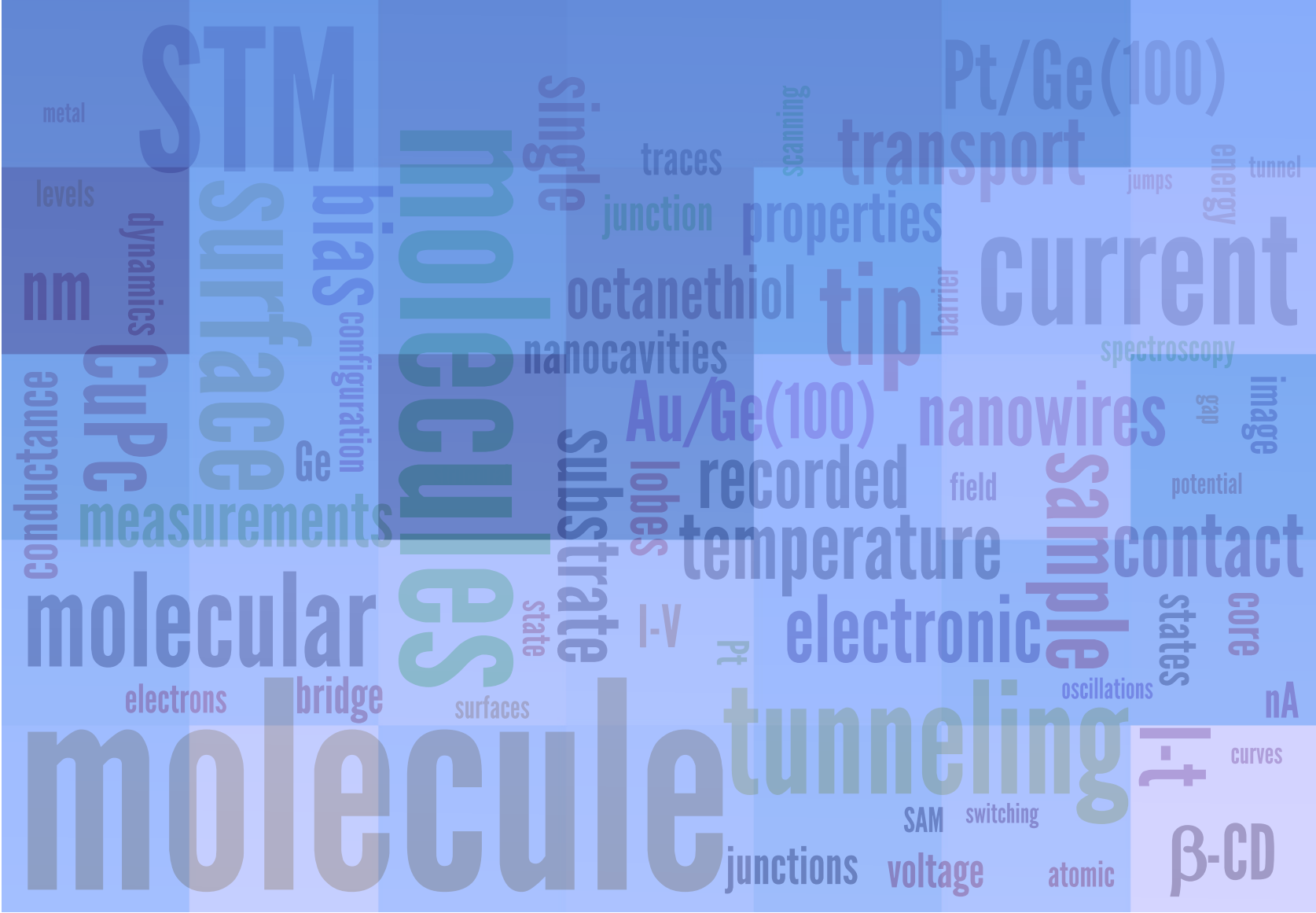




\section{The Art of Catching and Probing Single Molecules}




\section{Committee Members:}

Chairman and Secretary:

Prof. Dr. G. van der Steenhoven

University of Twente

Supervisor:

Prof. Dr. Ir. H. J. W. Zandvliet

University of Twente

Members:

Prof. Dr. D. M. de Leeuw

MPI-P Mainz

Prof. Dr. U. Köhler

Ruhr-Universität Bochum

Prof. Dr. Ir. B. Poelsema

University of Twente

Prof. Dr. Ir. J. Huskens

University of Twente

Prof. Dr. Ir. A. Brinkman

University of Twente

Dr. A. van Houselt

University of Twente

The work described in this thesis was carried out at the Physics of Interfaces and Nanomaterials (PIN) group, MESA + Institute of Nanotechnology, University of Twente, the Netherlands. The Research was financially supported by the Netherlands Organization for Scientific Research (NWO/CW ECHO.08.F2.008).

Avijit Kumar

The Art of Catching and Probing Single Molecules

ISBN: 978-90-365-0527-7

DIO: $10.3990 / 1.9789036505277$

Published by Physics of Interfaces and Nanomaterials, University of Twente Typeset in $\mathrm{LAT}_{\mathrm{E}} \mathrm{X} \boldsymbol{\varepsilon}$

Printed by Gildeprint Drukkerijen

Cover: A word cloud generated from the text of the thesis using www.wordle.net

(C)Avijit Kumar, 2013

No part of this publication may be stored in a retrieval system, transmitted, or reproduced in any way, including but not limited to photocopy, photograph, magnetic or other record, without prior agreement and written permission of the publisher. 


\title{
THE ART OF CATCHING AND PROBING SINGLE MOLECULES
}

\section{DISSERTATION}

\author{
to obtain \\ the degree of doctor at the University of Twente, \\ on the authority of the rector magnificus, \\ Prof. Dr. H. Brinksma,
}

on account of the decision of the graduation committee,

to be publicly defended

on Thursday 26 September 2013 at 12:45 hrs

by

\section{Avijit Kumar}

born on 11 December 1981

in Hazaribag, India 
This dissertation has been approved by Prof. Dr. Ir. H. J. W. Zandvliet 


\section{Contents}

Contents

1 Introduction 1

1.1 Molecular Electronics . . . . . . . . . . . . . 2

1.2 Scanning Tunneling Microscope . . . . . . . . . . . 6 6

1.2.1 Basic Principle of Scanning Tunneling Microscopy . . . 8

1.2.2 Scanning Tunneling Spectroscopy . . . . . . . . . . . 10

1.3 Scope and Outline of the Thesis . . . . . . . . . . . 11

2 Experimental Setup 13

2.1 Omicron Low Temperature Scanning Tunneling Microscope . . 14

2.2 RHK Scanning Tunneling Microscope . . . . . . . . . . . 16

2.3 Other Tools . . . . . . . . . . . . . . . . . . 18

3 Modified Ge(001) Surfaces as Nanotemplates 19

3.1 Nanotemplates for Molecular Electronics . . . . . . . . . . 20

3.1.1 Pt Modified Ge(001) Surface . . . . . . . . . . . . 20

3.1.2 Au Modified Ge(001) Surface . . . . . . . . . . . . 22

3.1.3 Nanocavity Arrays on Pt/Ge(001) Surface . . . . . . . 24

3.2 Experimentation . . . . . . . . . . . . . 24

3.3 Results and Discussion . . . . . . . . . . . . . 25

3.3.1 Structural Properties . . . . . . . . . . . 25

3.3.2 Electronic Properties . . . . . . . . . . . . . . 27

3.3.3 Structure of the Nanocavities . . . . . . . . . . . 29

3.4 Conclusion . . . . . . . . . . . . . . . 30 
4 Transport Through a Single Octanethiol Molecule 31

4.1 Introduction . . . . . . . . . . . . . 32

4.2 Experimentation . . . . . . . . . . . . 34

4.3 Results and Discussion . . . . . . . . . . . . . 35

4.3 .1 Transport at $77 \mathrm{~K} \ldots \ldots \ldots \ldots$

4.3.2 Temperature Dependent Transport . . . . . . . . . 42

4.4 Conclusion . . . . . . . . . . . . . . . . . . 44

5 Electronic Properties of a CuPc Molecule in a "Molecular Bridge" Configuration 45

5.1 Introduction . . . . . . . . . . . . . . . 46

5.2 Experimentation . . . . . . . . . . . . . 47

5.3 Results and Discussion . . . . . . . . . . . . . 48

5.3.1 Structural and Electronic Properties . . . . . . . . . 48

5.3.2 Dynamics of CuPc Molecules . . . . . . . . . . . 54

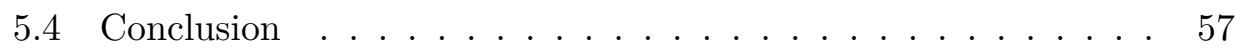

6 Electron Induced Dynamics of $\beta$-Cyclodextrin Molecules 55

6.1 Introduction . . . . . . . . . . . . . . . 60

6.2 Experimentation . . . . . . . . . . . . . . 62

6.2.1 Sample Preparation . . . . . . . . . . . . . 62 62

6.2.2 Time-resolved Scanning Tunneling Spectroscopy . . . . 62

6.3 Results and Discussion . . . . . . . . . . . . . . 63

6.3.1 $\beta$-Cyclodextrin Self-Assembled Monolayer . . . . . . . 64

6.3.2 Dynamics of $\beta$-Cyclodextrin Molecules . . . . . . . . . 65

6.3.3 Current-Voltage Oscillations in $\beta$-Cyclodextrin Monolayer 69

6.4 Conclusion .................. . . [72

\begin{tabular}{ll} 
Summary & 73 \\
\hline
\end{tabular}

Samenvatting $\quad 77$

References 81

Publication List

Acknowledgements 93 


\section{Chapter 1}

\section{Introduction}

"Molecular electronics" refers to using single molecules as active electronic devices such as diodes, transistors, memories etc. For physical realization of molecular electronics, a good understanding of the electronic transport properties of molecules is required. In this regard, the foremost ambition is to achieve a controlled and well-defined contact to a single molecule using macroscopic electrodes, and subsequently, to understand the structural and electronic properties of such molecular junctions. Scanning tunneling microscopy is a useful and an elegant technique to image single molecules and molecular assemblies and to establish a contact to them using an atomically sharp tip. As the title suggests, this thesis describes several studies made to catch or isolate single molecules or molecular assemblies to investigate their electronic properties using scanning tunneling microscopy. In this chapter, we provide brief descriptions of molecular electronics and scanning tunneling microscopy which has been used to perform the experiments presented in this thesis. 


\section{$1.1 \quad$ Molecular Electronics}

"Molecular electronics" was proposed in 1970s by Aviram and Ratner to use single molecules as active electronic components such as diodes, transistors, switches etc (1). Nearing a dead end of the Moore's law (2) has triggered a huge interest in molecular electronics since more than a decade. Even though there has been a tremendous amount of work carried out in this field, we are still faced with tough challenges and therefore a complete understanding of electronic transport properties of molecules is still lacking. The first challenge is to contact a single molecule using macroscopic electrodes in a well-defined fashion creating a metal-molecule-metal junction (3/5). The second challenge is related to the study of electronic transport properties of such metal-molecule-metal junctions as their electronic properties are substantially different than those of isolated molecules (5, 6).

Addressing the first challenge to create well-defined metal-molecule-metal junctions, various techniques were developed to establish contact to single molecules or molecular assemblies with macroscopic electrodes. Some of the techniques are based on break junctions such as mechanically-controlled break junctions (MJB) (9, 10), electromigrated break junctions (EBJ) (11), and scanning tunneling microscopic break junctions (STM-BJ) (4). Generally speaking, each of these techniques makes use of a pair of atomically sharp electrodes separated by a gap of few nanometers which can be bridged by molecule(s) to create metal-molecule(s)-metal junctions. Electronic transport properties of these molecular junctions are investigated by recording current-voltage measurements of the junctions. A schematic of MBJ technique is illustrated in Fig. 1.1(A). An MBJ consists of a metal bridge suspended in an insulating flexible substrate with a few nanometers size constriction at the middle of the bridge (9). The constriction is prepared by electron beam lithography or by making a notch in the metal bridge. As the substrate is bent mechanically, the metal bridge gets elongated before it breaks down at the constriction creating two atomically sharp electrodes opening a gap between them. The two electrodes can be opened and closed mechanically and also the size of the gap can be controlled very accurately. The molecules are placed to bridge the gap creating a metal-molecule(s)-metal junction as shown in Fig. 1.1(A). EBJ is very similar to MBJ except for the fact that the gap is created by an electromigration process using a high density current through the metal bridge (11). 

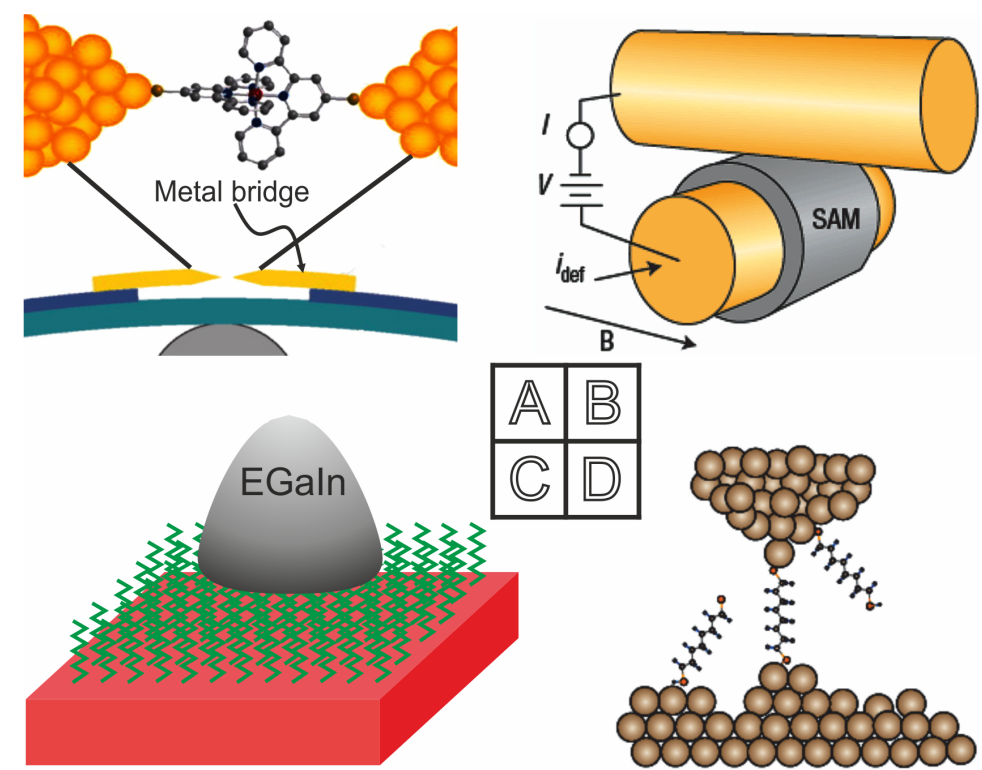

Figure 1.1: Schematics of various techniques employed to prepare metalmolecule(s)-metal molecular junctions. (A) In a mechanically-controlled break junction, two atomically sharp electrodes are prepared by mechanical deformation and molecules bridge the gap (adapted from (7)). (B) In a cross-wire junction, the molecules in a SAM are trapped between two crossed-wires and the separation between them is controlled by passing a current $i_{d e f}$ in a magnetic field $B$ to achieve the contact (adapted from (8) ). (C) In a liquid-metal drop method, eutectic GaIn acts as the top electrode on a self-assembled monolayer of molecules. (D) A scanning tunneling microscope break junction showing some molecules bridge the gap between an STM tip and a substrate (adapted from (8)).

These two techniques also provide an opportunity to use a gate electrode like a three point device. However, these techniques suffer from an uncertainty of the number of molecules bridging the gap and the geometries of the contacts (12). Therefore, a statistical approach is adapted during the measurements and the data analysis.

Another type of molecular junctions is based on self-assembled monolayers (SAM) of molecules deposited on a metallic substrate. Here, molecular junctions are prepared using techniques such as cross-wire junctions (13), conductive probe atomic force microscope (CP-AFM) junctions (14), and liquid-metal drop method. A cross-wire junction consists of two metal wires positioned in a cross geometry (13), one of the wires contains a SAM as shown in Fig. 1.1(B). The separation between the two crossed wires is controlled very pre- 
cisely using Lorentz force such that the other wire makes contact to the SAM. For CP-AFM junctions, a conducting probe AFM tip is brought in contact with a SAM, where the normal force feedback circuit of the AFM controls the mechanical load on the microcontact while the current-voltage characteristics are recorded (14). As shown in Fig. 1.1(C), metal drop methods such as eutectic GaIn (EGaIn) drop (15) or mercury drop (16) are used as a top electrode which provides a homogeneous, non-damaging, and conformal top contact to the molecular assembly prepared on substrate (15). EGaIn is an eutectic mixture of Ga and In with a melting point of $15.5^{\circ} \mathrm{C}$, just below the room temperature. As the EGaIn forms a conformal, non-toxic, micrometersized contact to SAMs surfaces, it was used to study the transport properties of $\beta$-cyclodextrin SAM (17). In these techniques the transport is averaged out and again statistical approach is used for the data analysis due to the presence of an ensemble of molecules between the two electrodes.

A powerful and versatile technique to contact molecule(s) is based on scanning tunneling microscope (STM). An STM has the capability to image single molecules or molecular assembly on a surface and study their electronic (transport) properties using scanning tunneling spectroscopy (STS). In the next section we provide a brief description of STM and STS. An STM break junction (STM-BJ) is created by moving an STM tip into contact with a conductive surface in a solution containing the desired molecules (4, 18). During the contact the molecules bridge the gap between the tip and the substrate. Subsequently, the tip is pulled out until the contact between the tip and the substrate breaks down and only molecule(s) is trapped between them. A schematic diagram of STM-BJ is shown in Fig. 1.1(D). STM is also used to study the electronic transport properties of SAM on surfaces by pushing the STM tip in the monolayer (19). These studies also involve an uncertain number of molecules in the molecular junction requiring a statistical analysis. To reduce the uncertainty a mixed monolayer of two different molecules is used (20). A high conductance molecules are grafted on an self-assembled monolayer of lower conductance molecules creating a mixed monolayer such that the single molecules of higher conductance can be studied by STM.

Using a sharp tip in STM, a single molecule junction is created by lifting a single molecule from the surface and trapping it between the tip and the surface (21) 23). In this case the transport occurs through a single molecule. An STM is also convenient to define electrodes with atomic precision by creating 
clusters on the surface as Schull et al. (24) did for studying transport through $\mathrm{C}_{60}$ molecules in contact with copper atoms. For large, disc shaped molecules like copper phthalocyanine lying flat on a surface (6), STM can be used to investigate Kondo resonance (25), switching, and dynamics (26) of molecules.

A complete understanding of electronic transport properties of metalmolecule-metal junctions has been elusive because of their complicated nature as they depend on the quality, composition, conformation and size of the junctions and metal-molecule interfaces (27). Charge transport through a molecular junction can be governed by several mechanisms (regimes) such as coherent transport, incoherent transport, hopping, Fowler-Nordheim tunneling etc (5).

In the coherent tunneling transport regime, the electrons tunnel through a potential barrier formed at the molecular junction due to the Fermi energy of the metal electrodes lying within the large gap of the highest occupied molecular orbital (HOMO) and lowest unoccupied molecular orbital (LUMO) of the molecule. The coherent tunneling process is valid for molecules with length $L$ smaller than $L_{m}$ and $L_{\phi}$ when a bias voltage smaller than the barrier height (low electron energy) is applied across the molecular junction. Here, $L_{m}$ and $L_{\phi}$ are the momentum coherence and phase relaxation lengths of the electrons, respectively. In this case, the conductance, $G$, of a molecular junction is related to the length $L$ of the molecule as:

$$
G \propto e^{-\beta L} .
$$

Here, $\beta$ is the current decay constant, ranging between 7 and $9 \mathrm{~nm}^{-1}$ for alkanethiol molecules and between 2 and $5 \mathrm{~nm}^{-1}$ for $\pi$-conjugated molecules (28). The coherent tunneling mechanism is temperature independent. A clear manifestation of the coherent tunneling mechanism in an octanethiol molecule has been presented in chapter 4 .

When the bias across the electrodes exceeds the barrier height, the potential barrier effectively changes from a trapezoidal to triangular shape. Here, the electrons tunnel through a triangular barrier and the process is described by Fowler-Nordheim tunneling. This is also known as field emission regime. Transition voltage spectroscopy is believed to describe the transition from the coherent tunneling to Fowler-Nordheim tunneling in molecular or vacuum junctions (29).

The incoherent tunneling or diffusive tunneling describes the transport of 
the electrons by tunneling through a series of potential wells in molecules of a typical length $L \geq L_{m}, L_{\phi}$. One can describe the transport as a series of coherent tunneling events where the electron sits in the potential wells for a short (residence) time. This process allows the electrons to tunnel through molecules as long as $4 \mathrm{~nm}(28)$. This process is temperature independent.

On the other hand, in the hopping conduction electrons "jump" over the potential barrier. In the hopping mechanism, current, $I$, is given by the classical Arrhenius relation (5)

$$
I \propto \exp \left(-\frac{E_{a}}{k_{B} T}\right),
$$

where $k_{B}$ is Boltzmann constant, $T$ is the temperature, and $E_{a}$ is the activation energy. This process is thermally induced as the jumping process involves an activation energy.

For a short molecule we expect coherent tunneling to be the dominant transport mechanism, whereas for large molecules, it is likely that the electrons tunnel incoherently through the molecule or follow hopping mechanism (28). A transition from the coherent tunneling to hopping regime has been observed in $\pi$-conjugated systems such as oligophenyleneimine (OPI) as the length of the molecule is increased. For short molecules (OPI 1 to 4) the resistance shows an exponential behavior, while for longer molecules (OPI 6 to 10) the resistance has a linear increase. A temperature-dependent study also showed the resistance of the shorter molecules is independent of temperature while for longer molecules it follows Arrhenius relation (28).

\subsection{Scanning Tunneling Microscope}

Scanning tunneling microscope (STM) was invented by Binnig and Hohrer (30) at IBM Zurich, for which they received the Nobel prize in 1986. STM is used in carrying out atomic resolution imaging, atomic manipulation (31) and studying local electronic and magnetic properties of conducting and semiconductor surfaces (32). It is said to be the precursor of atomic force microscope (AFM), both of which have played an important role in the development of nanotechnology (33). A variety of other interesting things can also be done using STM such as imaging chemical bonds between two atoms (34), identification of bonds (35), manipulation of chemical bonds (35, 36), dynamics of 
atoms (37) or molecules on the surfaces (38).

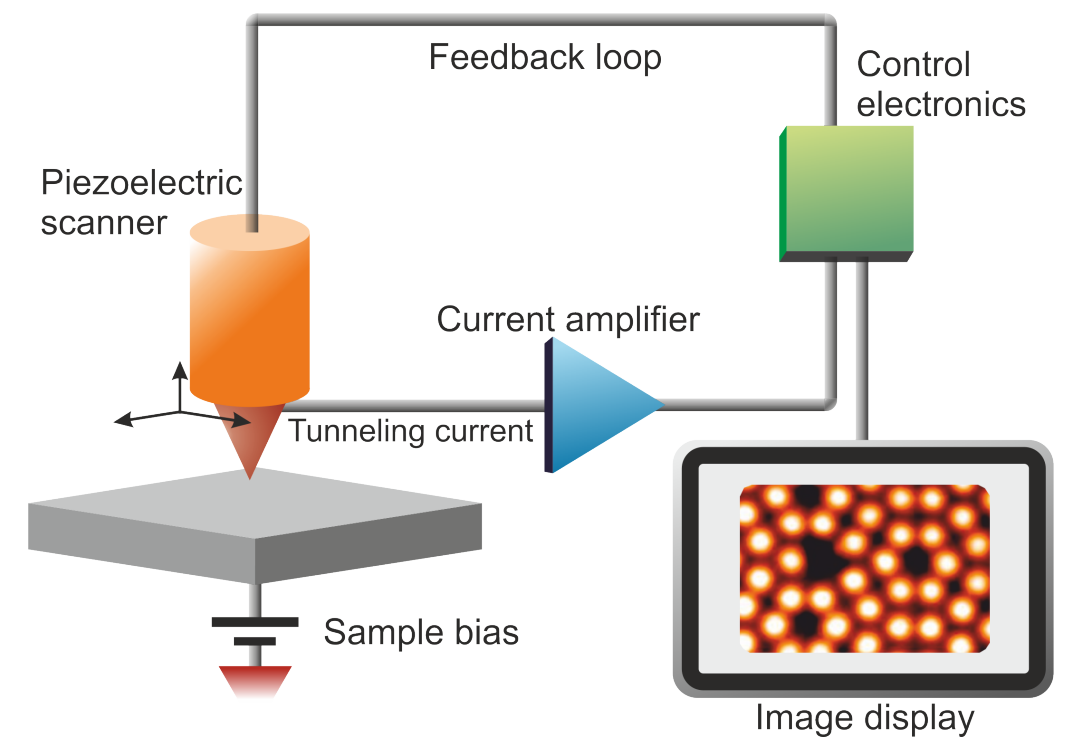

Figure 1.2: An schematic of an STM showing an atomically sharp tip placed at a distance $\sim 1 \mathrm{~nm}$ away from a conductive surface. The sample bias applied across the tip and the surface gives rise to a tunneling current. In the constant current mode, the tip scans the surface while the tunneling current is maintained constant by keeping the distance between the tip and the surface constant using the feedback loop. The movement of the tip is recorded as a function of the lateral position on the surface which gives rise to a contrast in the $z$-scan image.

In an STM, an atomically sharp metal tip is brought at a distance of $\sim 1$ $\mathrm{nm}$ away from a conductive surface and scanned over it to generate a three dimensional image. An schematic of an STM is shown in Fig. 1.2. An STM works on the principle of quantum mechanical tunneling of electrons between the atomically sharp tip and the substrate through a vacuum barrier. As shown in Fig. 1.2, a sample bias voltage $(\sim 1 \mathrm{~V})$ is applied across the tip and the sample giving rise to a tunneling current $(\sim 1 \mathrm{nA})$ which depends exponentially on the barrier width (the separation between the tip and the surface). This gives STM a very high resolution in the $z$-direction, normal to the surface. A lateral resolution of $0.1 \mathrm{~nm}$ and a vertical resolution of $0.01 \mathrm{~nm}$ can easily be accomplished. The vertical movement of the tip is controlled by piezoelectric elements through a feedback loop based on the tunnel current as shown in the figure. There are two modes of working with STM: the constant current mode which is widely used and the constant height mode. In the constant 
current mode, the distance between the tip and surface is kept constant using the feedback loop (for keeping the tunneling current constant) while the tip is scanning over. The vertical movement of the tip as a function of the lateral position on the surface is recorded and this gives rise to a contrast in the $z$-scan image. In the constant height mode, the surface is scanned at a fixed $z$-position (constant height) of the tip giving rise to a contrast in the current scan image. A brief introduction to the basic principle of STM is presented in the following section.

\subsubsection{Basic Principle of Scanning Tunneling Microscopy}

An STM works on the principle of quantum mechanical tunneling of electrons between a sharp tip and a substrate. Since an exact expression for the tunneling current is difficult to provide due to complicated wave functions of the less-known tip geometry and the surface, many approximations have been made for the tunneling current and for interpreting STM images. In the scattering approach, a tunneling current is obtained by solving Schrödinger equation for an incoming wave being reflected and transmitted due to the potential barrier between the tip and the surface. Simmons gave the solution for one-dimensional barrier using the Wentzel-Kramers-Brillouin (WKB) approximation and a free electron metal (39). For low sample bias voltages, the tunneling current, $I$, is approximated as

$$
I \propto \frac{V}{z} e^{-2 \kappa_{0} z},
$$

where $V$ is the applied voltage; $z$ is the barrier width; and $\kappa_{0}$ is the decay constant of the wave function in the barrier $\left(\frac{\hbar^{2} \kappa_{0}^{2}}{2 m}=\phi-\frac{e V}{2} ; \phi\right.$ is the barrier height). The important feature of the above equation is the exponential dependence of the tunneling current on the barrier width, the reason for a very high resolution in the $z$-direction.

However, physical interpretation of STM images is more complicated. In the simplest case, the tunneling current is proportional to the electron density due to all electronic states of the surface at the tip apex region. The wave function of an electron, $\psi_{s}(z)$, within a rectangular potential barrier (normal to the surface) can be given as (32),

$$
\psi_{s}(z)=\psi_{s}(0) e^{-\kappa z}
$$


Here, $\kappa=\sqrt{2 m(U-E)} / \hbar$ is the decay constant and $z$ is the position within the potential barrier. $U, E$, and $m$ are the height of the potential barrier, energy of the wave function of the electron, and electronic mass, respectively. The total current can be deduced by summing up over all the electronic states of the surface (32, 40),

$$
I=\sum_{E_{f}-e V}^{E_{f}}\left|\psi_{n s}\left(r_{0}\right)\right|^{2} e^{-2 \kappa z} .
$$

$\psi_{n s}\left(r_{0}\right)$ is the wave function of the $\mathrm{n}^{\text {th }}$ state of the surface at location $r_{0}$; $V$ is the bias applied across the tip and the substrate and $E_{f}$ is the Fermi energy. If $V$ is small enough then the density of electronic states does not vary significantly and the right hand side of the last equation can be written in terms of the local density of states (LDOS) of the surface at $r_{0}$ at the Fermi level 1 . Therefore, the tunneling current can be written in terms of LDOS as,

$$
I \propto V \rho\left(r_{0}, E_{f}\right) e^{-2 \kappa z} .
$$

A more quantitative theory of the tunneling current was given by TersoffHamann (41) and later by Chen (42) which was based on Bardeen's theory. Bardeen's theory is considered to be accurate for a tip sufficiently far away from the sample and for a low enough sample bias. Based on the scattering theory, he calculated tunneling current using effective transfer matrix. However, to evaluate effective matrix element one must know the wave functions of the tip and the sample. The wave function of the outermost atom of the tip was modeled to be an $s$-wave function by Tersoff-Hamann (41) and then the tunneling current was given by

$$
I \propto V N_{t}\left(E_{f}\right) \operatorname{LDOS}\left(r_{0}, E_{f}\right),
$$

where $N_{t}\left(E_{f}\right)$ is the density of states of the tip at the Fermi energy. However, it failed to explain a higher value of atomic corrugations measured on the metal surface than predicted by this model. Later on Chen used a more directional tip orbitals such as $p_{i}(i=x, y, z), d_{z^{2}}$ etc, to model the wave function of the tip (42) which allowed a better prediction of experimentally observed corrugations.

\footnotetext{
${ }^{1} \mathrm{LDOS}, \rho(z, E) \equiv \frac{1}{\epsilon} \sum_{E_{n}=E-\epsilon}^{E}\left|\psi_{s}(z)\right|^{2}$ for sufficiently small $\epsilon$
} 


\subsubsection{Scanning Tunneling Spectroscopy}

Besides imaging, an STM is also used for extracting electronic information of conductive and semiconductor surfaces and this technique is commonly known as scanning tunneling spectroscopy (STS) (43). This is essential for investigation of electronic transport properties of single molecules or molecular assemblies. There are three well-known spectroscopy techniques as described briefly.

Current-voltage (I-V) spectroscopy, which is used to determine the electronic properties of a surface. At a fixed tip-surface distance, the tunneling current is recorded as the sample bias is modulated. A metal surface shows a linear I-V curve with finite slope at zero bias (Fermi energy of the surface) while a semiconductor surface shows no current around zero bias due to the presence of a band gap. The I-V spectroscopy is used to extract differential conductance $\left(\frac{d I}{d V}\right)$, local density of states $\left(\frac{d I}{d V} / \frac{I}{V}\right)$ etc. At negative sample bias, electrons tunnels from the surface to the tip which allows to investigate LDOS of the filled states while at positive sample bias the electrons tunnel into the sample allowing us to investigate LDOS of the empty states of the surface.

Time-resolved current (I-t) spectroscopy, which is used to investigate dynamic events on a surface. The tip is placed at a fixed height by keeping the feedback loop off while the tunneling current is recorded against time. Any dynamic event on the surface in the proximity of the STM tip is reflected as a change of the tunneling current in the current-time plot. Generally, the temporal resolution of STM is in the order of seconds. However, I-t spectroscopy enhances the temporal resolution to few $\mu$ s and is limited by the bandwidth of the current amplifier.

Current-distance (I-z) spectroscopy, which is used to estimate the barrier height of the junction and the work function of a surface. Here, the tunneling current is recorded as the STM tip is approached to or retracted from the surface. The barrier height between the tip and the surface is extracted from the exponent of the tunneling current plotted against the tip-surface distance.

Existence of such diverse spectroscopy techniques in combination with atomic resolution imaging make STM a powerful technique in the field of molecular electronics. 


\subsection{Scope and Outline of the Thesis}

This thesis deals with probing electronic or electronic transport or dynamic properties of single molecules or molecular assemblies immobilized on various substrates using STM and STS.

Chapter 2 briefly describes the experimental setups used for probing single molecules or assemblies immobilized on various surfaces. An Omicron lowtemperature STM setup and an RHK variable temperature STM setup have been described.

Chapter 3 is devoted to nanotemplates based on modified Ge(001) surfaces. Apart from perfectly straight atomic chains present on the $\mathrm{Pt} / \mathrm{Ge}(001)$ surface, we also observed an array of self-organized nanocavities. We study structural and electronic properties of the nanocavities using STM and STS.

Chapter 4 is focused on electronic transport through a single octanethiol molecule trapped between a Pt/Ge(001) substrate and an STM tip. The octanethiol molecule flips to bridge a $\sim 1 \mathrm{~nm}$ gap between the substrate and the STM tip when the electric field between them exceeds a threshold value. Furthermore, we demonstrate that either tunneling or ballistic transport is the main transport mechanism in an octanethiol molecule.

Chapter 5 presents a study of structural and electronic properties of copper phthalocyanine $(\mathrm{CuPc})$ molecules in a molecular bridge configuration. The molecular bridge configuration is realized by trapping a $\mathrm{CuPc}$ molecule between two adjacent $\mathrm{Au} / \mathrm{Ge}(001)$ nanowires so that the copper core is decoupled from the substrate. The STM measurements show that the $\mathrm{Cu}$ core is dim at low sample biases, but becomes suddenly bright at a bias larger than 3.5 V. Time-resolved current spectroscopy recorded on lobes with speckles show a lateral dynamics of the molecules on the surface.

Chapter 6 describes variable-temperature STM and STS measurements on hepthathioether $\beta$-cyclodextrin ( $\beta$-CD) self-assembled monolayers (SAM) on $\mathrm{Au}(111)$ surface. The $\beta-\mathrm{CD}$ molecules exhibit very rich dynamical behavior which is not observed in an ensembled averaged studies performed using macroscopic junctions such as metel-drop eutectic GaIn technique (EGaIn). 


\section{Chapter 2}

\section{Experimental Setup}

Scanning tunneling microscopy (STM) is used to carry out atomic scale imaging, atomic manipulation and to study local electronic and magnetic properties of conducting surfaces. Because of these properties and the fact that an atomically sharp STM tip can be used as an electrode for establishing contact to a single molecule, it has become an essential tool in the field of molecular electronics. For the work carried out in this thesis, we have used two STMs: an Omicron Low temperature STM and an RHK UHV700 variable temperature STM. In this chapter a brief introduction to these STM setups will be provided. 


\subsection{Omicron Low Temperature Scanning Tunneling Microscope}

The Omicron low temperature scanning tunneling microscope (LT-STM) is a commercial low temperature STM procured from Omicron NanoTechnology GmbH, Germany. The STM works at ultrahigh vacuum (UHV) conditions at liquid nitrogen $\left(\mathrm{LN}_{2}\right)$ and liquid helium $(\mathrm{LHe})$ temperatures. As shown in Fig. 2.1. the STM consists of a main chamber where the STM scanner is located, a home-built preparation chamber for processing of the samples and a load lock unit to introduce samples. The preparation chamber is equipped with a sputter gun, a heating stage, a Pt evaporator, a Kundsen cell evaporator, a leak valve for depositing molecules and a commercial mass spectrometer. The system is also equipped with two magnetic transfer rods to transfer samples from the load lock to the preparation chamber and then to the STM chamber. The base pressure in both the main chamber and the pressure chamber is maintained below $5 \times 10^{-11}$ Torr. The purity of the gaseous contents is monitored by the mass spectrometer. It is also used for leak detection in the chambers. The experiments described in chapters $3,4,5$ and 6 are performed using this system.

The STM consists of a single piezo tube scanner (shown on the left panel of Fig. 2.3 which provides $z$-resolution better than $0.01 \mathrm{~nm}$. The $z$-axis refers to the direction normal to the surface. The whole scanner stage is enclosed inside two concentric bath cryostats. The outer one is filled with $\mathrm{LN}_{2}$ for thermal shielding and the inner one for cooling the scanner stage which is filled with either $\mathrm{LN}_{2}$ for measurements at $77 \mathrm{~K}$ or LHe for measurements at $4 \mathrm{~K}$. Low temperature measurements allow for a better resolution as the stability of the STM tip is greatly improved. Variable temperature measurements can be performed in two ways. One way is by using a counter heating element present inside the STM which works with $\mathrm{LHe}$ and $\mathrm{LN}_{2}$ cooling. The other way is by letting the temperature of the STM rise as the cryo-liquid has eventually evaporated. However, measurements at temperatures from $77 \mathrm{~K}$ onwards only can be performed using the latter method. It does not work in case of LHe because the rise in temperature is too fast and this creates a huge thermal drift during the measurements.

The sputter gun, located in the preparation chamber, is used to sputter the top layers of the substrates using high energy $\mathrm{Ar}^{+}$ions. $\mathrm{Ar}^{+}$ions are ionized 


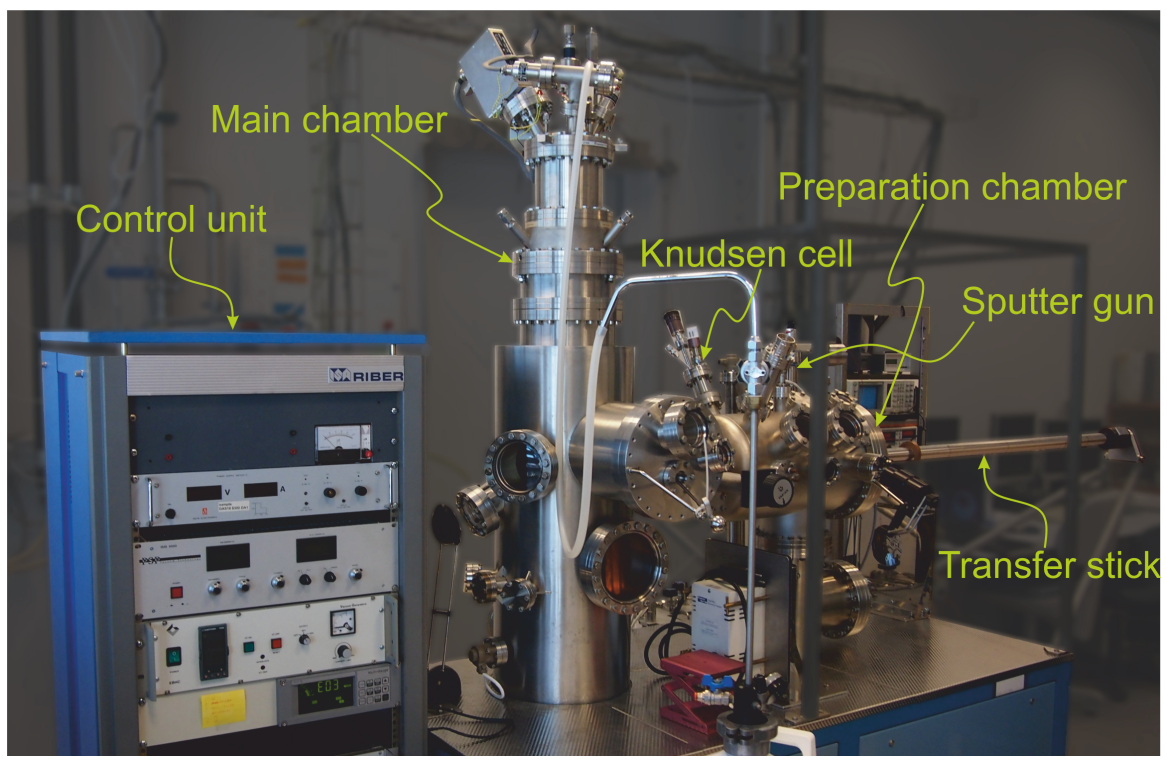

Figure 2.1: A photograph of the Omicron Low-temperature scanning tunneling microscope (LT-STM) setup used for the experiments carried out in this thesis. The system consists of an STM chamber, a preparation chamber, and a load lock unit.

at an Ar gas pressure of $5 \times 10^{-6}$ Torr and they are accelerated towards the surface with an energy of 500 or $800 \mathrm{eV}$. The $\mathrm{Ar}^{+}$beam is focused to enhance the ion flux on the sample surface and to avoid sputtering of the sample holder. An emission current of $9 \mu \mathrm{A}$ is normally used for sputtering the surface. In general, with the parameters used in our experiments, a sputter rate of 1-2 monolayer $/ \mathrm{min}$ is accomplished. The sample holders are made of Mo and Ta to avoid any contamination during sample handling and sputtering. A heating stage is used to anneal semiconductor substrates (for example Ge or $\mathrm{Si}$ ) using direct current resistive heating. This way sputtered surfaces are repaired to achieve atomically smooth terraces on them. Usually, a fast annealing (cooling) rate results in small terraces while a slower annealing rate results in larger terraces. A several repetitive cycles of $20 \mathrm{~min}$ of sputtering and annealing gives atomically flat clean surfaces. The heating stage is also used to anneal $\mathrm{Ge}(001)$ surfaces after metal (Pt or $\mathrm{Au}$ ) deposition.

We use an evaporator to deposit $\mathrm{Pt}(\mathrm{or} \mathrm{Au})$ on $\mathrm{Ge}(001)$ surfaces. This is a direct current resistive heating evaporator which is made of $\mathrm{W}$ wire (diameter: $0.37 \mathrm{~mm}$ ) with $\mathrm{Pt}$ (or $\mathrm{Au}$ ) wire wrapped around it. Applying a high current 
through the evaporators allows us to deposit $\mathrm{Pt}(\mathrm{or} \mathrm{Au}$ ) with submonolayer coverage. While the $\mathrm{Pt}$ evaporator is located in the preparation chamber, the $\mathrm{Au}$ evaporator is located in the load lock.

A leak valve is used for depositing molecules with a high vapor pressure. We have deposited octanethiol molecules (melting point: $-49{ }^{\circ} \mathrm{C}$ ) on $\mathrm{Pt} / \mathrm{Ge}(001)$ surfaces using the leak valve as described in chapter 4. A Kundsen cell evaporator is used to deposit molecules with lower vapor pressure. We have used it for depositing copper phthalocyanine molecules (melting point: $350{ }^{\circ} \mathrm{C}$ ) on Au modified Ge(001) surfaces as described in chapter 5 .

\subsection{RHK Scanning Tunneling Microscope}

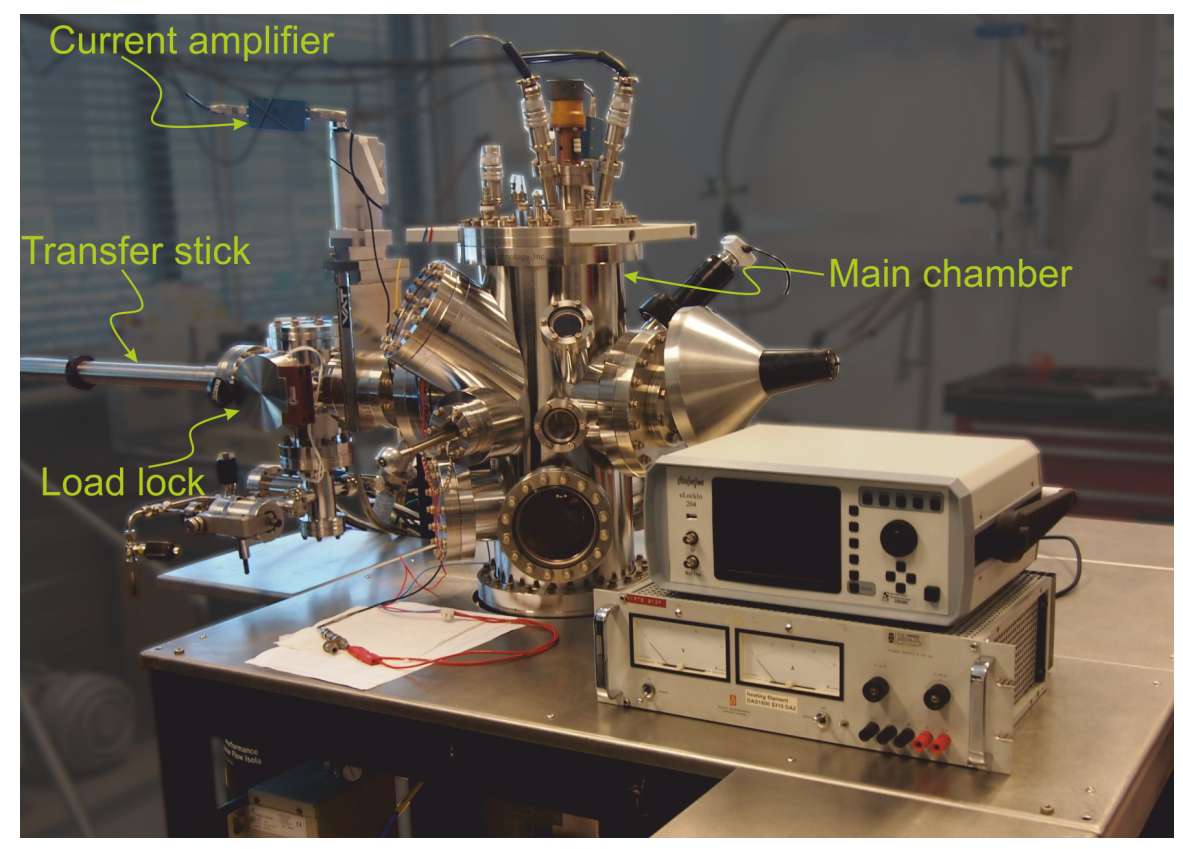

Figure 2.2: A photograph of the RHK scanning tunneling microscope (STM) setup used for the experiments carried out in this thesis. The system consists of an STM chamber, and a loadlock unit. The current amplifier is located outside the main chamber allowing one to substitute it with other amplifiers of desired gain.

The RHK UHV700 is a variable temperature UHV STM which works in the temperature range from $90 \mathrm{~K}\left(\mathrm{LN}_{2}\right)$ up to $1000 \mathrm{~K}$ (by heating using a 
filament). As shown in Fig. 2.2 the system consists of a main chamber for measurement and a load lock unit for introducing samples. The base pressure in the main chamber is $1 \times 10^{-10}$ Torr, while the pressure in the load lock is $5 \times 10^{-9}$ Torr. The STM consists of a Variable Temp Beetle ${ }^{\mathrm{TM}}$ type scanner. As the scanner is thermally isolated from the sample stage and the sample holder, it provides a great flexibility to carry out measurements over a large temperature range. The scanner and the sample stage are shown on the right panel of Fig. 2.3. The setup is used to carry out the experiments described in chapter 6 .

The current amplifier of the STM is located outside the main chamber. This makes the system very appropriate for large current measurements (for example transport studies) by substituting the current amplifier with one of lower gains. An example of a variable gain current amplifier is DLPCA-200 (gain range: $1 \mathrm{kV} / \mathrm{A}$ to $100 \mathrm{GV} / \mathrm{A}$ ) procured from Femto, Germany.
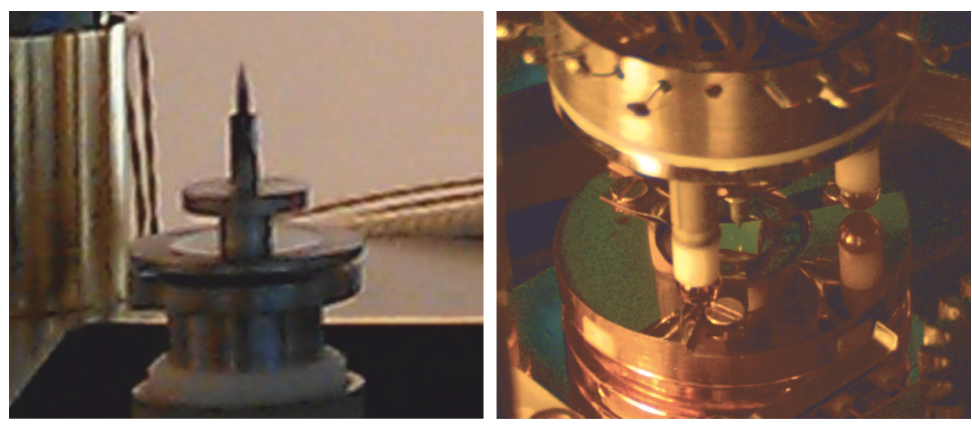

Figure 2.3: Photograph on the left panel shows a single piezo tube scanner from the Omicron LT-STM. The tip is pointing upwards. The right photograph shows a Variable Temp Beetle ${ }^{\mathrm{TM}}$ type scanner placed over a sample holder from the RHK STM. Here, the tip is pointing downwards.

The sample holder is either equipped with a filament for electron bombardment or with a provision for direct current heating of semiconductor samples. High temperature STM measurements can be performed by heating the sample using the filament. Also, temperatures as low as $90 \mathrm{~K}$ can be achieved using a flow cryostat unit using $\mathrm{LN}_{2}$. A K-type chromel-alumel alloy thermocouple located in the sample holder can measure the temperature of the sample. 


\subsection{Other Tools}

We have used tungsten (W) tips for all the measurements. W tips were prepared by electrochemical etchings of $\mathrm{W}$ wires with diameters of $0.25 \mathrm{~mm}$ and $0.37 \mathrm{~mm}$ for the RHK and the LT-STM, respectively. A $2 \mathrm{M} \mathrm{NaOH}$ solution was used as the electrolyte and a Pt ring as the counter electrode.

Experiments performed using the lock-in detection technique, such as described in chapter 3 and 5, were performed using a Stanford Research System SRS830 DPS lock-in amplifier. 


\section{Chapter 3}

\section{Modified Ge(001) Surfaces as Nanotemplates}

In this chapter, we present Ge(001) surfaces modified by Pt or Au as potential nanotemplates for immobilization of single molecules. Pt/Ge(001) and $A u / G e(001)$ surfaces contain regular arrays of nanowires with very interesting physical and electronic properties suitable for studying single molecules for molecular electronics. Here, we investigate structural and electronic properties of a new phase of ordered arrays of nanocavities on the Pt/Ge(001) surface using Scanning Tunneling Microscopy and Spectroscopy. The nanocavities are thermodynamically stable and form ordered domains separated by anti-phase boundaries. When imaged at negative sample bias, the nanocavities exhibit an elliptical shape, while they appear as rectangular cavities at positive sample bias. A well-defined electronic state at $0.7 \mathrm{eV}$ above the Fermi level is found in the $\frac{d I}{d V}$ spectrum. Spatial maps of the differential conductivity reveal that this electronic state is located at the regions between neighboring nanocavities of the ordered arrays. 


\subsection{Nanotemplates for Molecular Electronics}

As down-scaling of electronic devices is reaching the atomic limit, a thorough and detailed study of the properties of single molecules and nanostructures is an absolute necessity. A proper understanding will pave the way to novel applications in the field of molecular electronics, nanoelectronics and quantum computing. At the nanometers length-scale conventional lithographic processes are not suited to produce nanotemplates or arrays for the immobilization and study of individual molecules (44). Therefore, it becomes important to explore material systems or processes for fabricating nanotemplates where single molecular entities can be trapped. Such material systems are also relevant for providing nucleation centers, which can be beneficial for highly selective catalysis applications (45). This is one of the key reasons why we have been witnessing a large number of studies on self-assembly and self-organized growth of nanostructures during the last two decades (44, 46 51).

The realization of self-assembly of nanostructures using chemical synthesis using elementary building blocks (tectons) has revealed a cornucopia of new and exciting science (47, 52, 53). The weak non-covalent interactions among the tectons can be tuned to yield tailored structures and patterns with desired properties. However, in the case of self-organized growth of nanostructures using inorganic materials, such as metals and semiconductors, the scope and control for tailoring these structures becomes rather limited because these materials exhibit very strong directional (in semiconductors) or non-directional bonds (in metals) (46). This poses a difficulty to manipulate the inter-atomic interactions leading to organized structures. Some examples of semiconductor or/and metal self-organized structures are nanoripples on metals (54, 55), nanogrooves on $\mathrm{Cu}(001)$ (56), quantum dots of $\mathrm{Ge}$ on $\mathrm{Si}(001)$ (46) and arrays of atomic wires on $\mathrm{Ge}(001)$ (49, 57 5 59$)$. In the following sections we present brief descriptions of nanowires formed on $\mathrm{Ge}(001)$ surface by depositing on it $\mathrm{Pt}$ or $\mathrm{Au}$. Because they possess a rich structural and electronic properties we have used them as template to investigate properties of single molecules for molecular electronics as described in chapters 4 and 5.

\subsubsection{Pt Modified Ge(001) Surface}

The Pt modified $\mathrm{Ge}(001)$ ( $\mathrm{Pt} / \mathrm{Ge}(001)$ ) surface has been studied for quite some time because of the presence of well-ordered atomic wires on the surface 
which exhibit various interesting quantum effects. The surface is prepared by depositing a fraction of a monolayer of $\mathrm{Pt}$ on a clean $\mathrm{Ge}(001)$ surface followed by subsequent annealing $(49,57)$. This modified surface consists of $\alpha$-terraces, $\beta$-terraces, atomic wires and arrays of nanocavities on $\beta$-terraces. Because of its appealing structural and electronic properties this system serves as an excellent nanotemplate and was used previously to study single molecules for molecular electronics(21, 60).

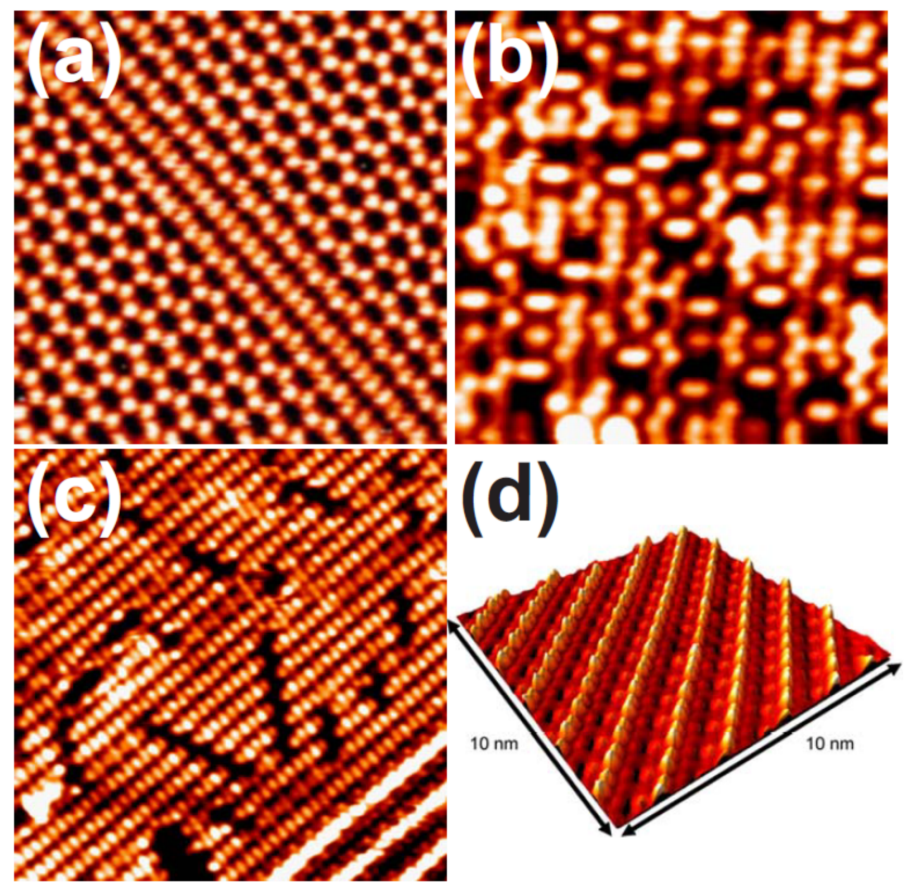

Figure 3.1: STM images of (a) a clean Ge(001) surface consisting of $(2 \times 1)$ and c $(2 \times 4)$ reconstructions, (b) disordered $\alpha$-phase consisting of $2 \times 1$ missing dimers. They are characteristic of presence of metals on the (sub)surface of a semiconductor surface, (c) $\beta$-terrace consisting of ordered missing dimer defects in [310] and [110] directions. They form a bed for the formation of nanowires as shown in the bottom right corner. (d) perfectly straight nanowires of an atomic diameter with interseparation of $1.6 \mathrm{~nm}$. [adapted from (49)]

The various phases/terraces on Pt/Ge(001) surface are shown in Fig. 3.1 (a) clean Ge surface, (b) $\alpha$-terrace, (c) $\beta$-terrace, and (d) nanowires. The $\alpha$ terrace comprises symmetric (Ge-Ge) and asymmetric Ge (Ge-Pt) dimers in addition to a high density of missing dimers defects. These defects can be identified as $2+1$ missing dimer defects (two missing dimer defects followed 
by one dimer and a missing dimer defect) (49). The $\beta$-terrace is identified with the presence of dimer vacancy lines aligned along $<310>$ and $<110>$ directions. The $\beta$-terrace consists of nanowires which are defect free, straight and have a cross section of only one atom. The nanowires are oriented in [110] and $[1 \overline{1} 0]$ directions along the $\mathrm{Ge}(001)$ dimer rows. The separation between the nanowires is mainly $1.6 \mathrm{~nm}$ (sometimes $2.4 \mathrm{~nm}$ and rarely $3.2 \mathrm{~nm}$ ) which relates to integral multiple of the shortest distance between Ge atoms in the bulk in (001) plane.

A lot of interesting studies done on these nanowires have revealed interesting quantum phenomenon such as confinement of electronic states between the wires and a Peierls instability. The confined electronic states are located in the troughs between the nanowires and has been explained using a model of a particle in a one dimensional quantum well (61). As the size of the well, that is the separation between the nanowires, is increased the separation between the energy states goes down and more of such states can be accommodated between the nanowires. The nanowires consist of $\mathrm{Pt}$ atoms which is evident from the preferential adsorption of $\mathrm{CO}$ molecules on the nanowires. At room temperature the wires are in the form of Pt dimers as shown in Fig. 3.1(d). However at lower temperature, the Pt dimers undergo a Peierls transition and the 2-fold periodicity changes to 4 -fold periodicity (62). This structural transition is accompanied with an electronic transition where the metallicity of the nanowires is substantially reduced in accordance with Peierl's instability. Another beautiful observation of the $\mathrm{Pt} / \mathrm{Ge}(001)$ system is that it entails one of the smallest electromechnical device which resembles an atomic pinball machine (37). The central atoms of a dimerized Pt pair acts like flippers of the atomic pinball machine whose frequency can be controlled with amount of electrons injected through it. The various configurations of the flippers were identified by time-resolved current spectroscopy.

\subsubsection{Au Modified Ge(001) Surface}

Shortly after the discovery of Pt nanowires on Ge(001), Au induced Ge(001) were reported by Wang et al. (64) which was followed by an extensive investigation regarding its structural and electronic properties in the recent years (59, 63, 65 (73). $\mathrm{Au} / \mathrm{Ge}(001)$ surface is prepared by depositing a fraction of a monolayer of $\mathrm{Au}$ on a clean $\mathrm{Ge}(001)$ substrate followed by annealing at $650 \pm 25 \mathrm{~K}$. Very similar to the $\mathrm{Pt} / \mathrm{Ge}(001)$ nanowires, $\mathrm{Au} / \mathrm{Ge}(001)$ surface 

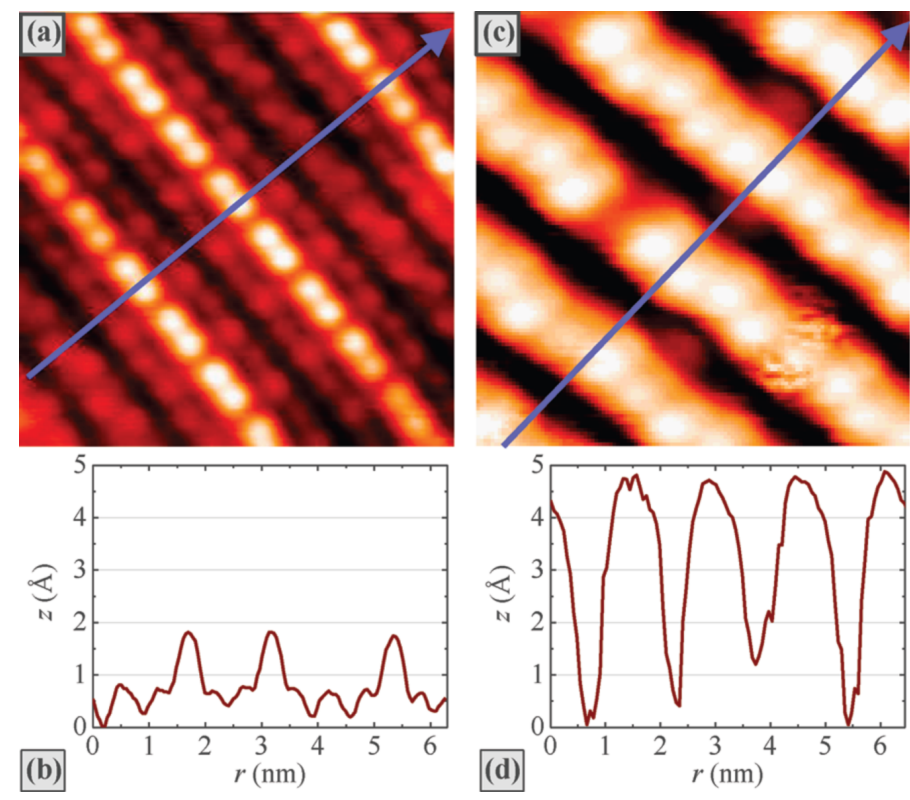

Figure 3.2: A comparison of STM images of $\mathrm{Pt} / \mathrm{Ge}(001)$ nanowires with $\mathrm{Au}$ induced $\mathrm{Ge}(001)$ nanowires. (a) shows Pt induced nanowires, (b) a line profile taken across the Pt nanowires along the blue line from image (a), (c) Au induced nanowires, (d) a line profile taken across the Au nanowires along the blue line from image (c). As can be seen from (b) and (d) that the corrugation across the Au induced nanowires is as large as $5 \AA$ compared to $1.5 \AA$ of $\mathrm{Pt}$ nanowires. Also, the ridges in (c) are relatively broader and contains Ge-Ge dimers at the top [adapted from $(63)]$.

consist of a regular arrays of straight, parallel, and kink free nanowires as shown in Fig. 3.2 They are also oriented in [110] and [1 $\overline{1} 0]$ directions and their length is limited by the size of terraces and the defects present on the surface. The separation between the two nanowires is $1.6 \mathrm{~nm}$. Even though they share few similarities with $\mathrm{Pt} / \mathrm{Ge}(001)$ nanowires, they differ from them in various aspects. The corrugation across $\mathrm{Au}$ nanowires are at least $6 \AA$ compared to only $1.5 \AA$ for Pt nanowires as it is shown in Fig. 3.2 (b) and (d). Such larger corrugation of $\mathrm{Au}$ nanowires indicates that its structure is more complicated that than of atomic chain of $\mathrm{Pt} / \mathrm{Ge}(001)$. Van Houselt et al. (59) gave the most agreeable structural model of $\mathrm{Au} / \mathrm{Ge}(001)$ nanowires so far, according to which, the nanowires are composed of Ge (111) and (1 11$)$ facets on alternate sides. These facets are decorated with an overlayer of $\mathrm{Au}$ with $(\sqrt{3} \times \sqrt{3}) \mathrm{R} 30^{\circ}$ reconstruction similar to Au-induced reconstruction 
on $\mathrm{Ge}(111)$ surface. This gives rise to nanowires corrugation of more than 6 $\AA$. The ridges of the nanowires show a zig-zag structure indicating that they consist of buckled Ge-Ge or Au-Ge dimer rows (59, 64).

Scanning tunneling microscopy (STM) and spectroscopy (STS) studies on $\mathrm{Au} / \mathrm{Ge}(001)$ surface show that both the nanowires and the troughs are metallic in nature (63, 65, 66, 74) and their differential conductivities are comparable (63, 66) at the Fermi energy. Blumenstein et al. (71) reported that the density of states of these nanowires show universal scaling of energy and temperature which is a characteristic one dimensional Tomonaga Lutting Liquid. By performing differential conductivity mappings, Heimbuch et al. (75) showed these states are located in the troughs between the nanowires instead of on the nanowires. With such structural and electronic properties in mind, these structures can serve as nanotemplates for studying molecular electronics. Berkelaar et al. deposited copper phthalocyanine molecules on $\mathrm{Au} / \mathrm{Ge}(001)$ surface and investigated their properties (76). More on this will be discussed in chapter 5 .

\subsubsection{Nanocavity Arrays on Pt/Ge(001) Surface}

We have found a relatively new phase of ordered arrays of nm-sized cavities on a $\mathrm{Pt} / \mathrm{Ge}(001)$ surface. The nanocavities are thermodynamically stable and exhibit structural and electronic properties that deviate substantially form the bare Ge(001) substrate. The nanocavities are similar to the cavities observed by Fischer et al. (49). However, in our case the density of nanocavities is much higher and they are closely packed into well-ordered arrangements, which give rise to new electronic effects. Given the multi-phase character and the rich properties of the $\mathrm{Pt} / \mathrm{Ge}(001)$ surface, it can provide a unique template for the study and characterization of single molecules or nanoclusters by controllably tailoring the phase and thereby the electronic and structural properties. In this chapter, we study physical, structural and electronic properties of this ordered arrays of nanocavities on the Pt/Ge(001) surface using STM and STS.

\subsection{Experimentation}

The experiments were performed in Ultra High Vacuum (UHV) conditions at room temperature and at $77 \mathrm{~K}$ in the Omicron Low Temperature Scanning Tunneling Microscope (LT-STM). The Ge(001) samples were cut from a nom- 
inally $76.2 \mathrm{~mm}$ (3 inches) by $0.5 \mathrm{~mm}$, single side-polished, n-type, Sb-doped wafer with a resistivity of less than $0.01 \Omega \mathrm{cm}$. The samples were mounted on a Mo holder and any contact between the samples and other metals was carefully avoided. The preparation of the samples was carried out in the preparation chamber at UHV conditions. The samples were cleaned by sputtering with $\mathrm{Ar}^{+}$ions with an energy of $800 \mathrm{eV}$ and subsequently annealed at a temperature of $1100 \pm 50 \mathrm{~K}$. We repeated this cycle for several times until we observed an ordered pattern of coexisting $(2 \times 1)$ and $c(4 \times 2)$ domains $(77,78)$. Subsequently, we deposited $0.2-0.5$ monolayers of $\mathrm{Pt}$ on this substrate followed by prolonged annealing at $1050 \pm 25 \mathrm{~K}$. After cooling down to room temperature the samples were transferred to the STM for imaging. The measurements were carried out at room temperature and at $77 \mathrm{~K}$ using electrochemically etched $\mathrm{W}$ tips. $\frac{d I}{d V}$ spectra and spatial maps of the differential conductivity were obtained using a lock-in amplifier. The modulation voltage was set to an amplitude 10-20 $\mathrm{mV}$ and the frequency was $8.4 \mathrm{kHz}$.

\subsection{Results and Discussion}

\subsubsection{Structural Properties}

We carried out STM and STS measurements on the Pt/Ge(001) samples that were prepared as described in the experimental section. Filled state images recorded at room temperature revealed that the surface contained ordered arrays of nanocavities. Subsequently, the sample was cooled down to $77 \mathrm{~K}$ and all the measurements were carried out. Figure 3.3 shows an STM image of such an area of size $80 \times 80 \mathrm{~nm}^{2}$ recorded at a sample bias of $+1.5 \mathrm{~V}$ and a setpoint current of $0.3 \mathrm{nA}$. The surface exhibits several domains of well-ordered and densely packed nanocavities, as shown in the outlined regions. The anti-phase boundaries mainly run along [310] and [310] directions as indicated by the arrows in Fig. 3.3 . The inset of Fig. 3.3 shows an STM image of nanocavities existing along with several $\mathrm{Pt}$ nanowires on the same terrace.

However, the amount of nanowires found on the surface was pretty low. A nanowire which terminates near a nanocavity is indicated by a small circle. Apart from the nanowires and the nanocavities, the $\mathrm{Pt} / \mathrm{Ge}(001)$ surface also consists of $\alpha$-terraces and $\beta$-terraces $(49,57)$. The formation of $\alpha$-, $\beta$-terraces, and nanowires is attributed to the amount of $\mathrm{Pt}$ that is locally available on the surface (57). 


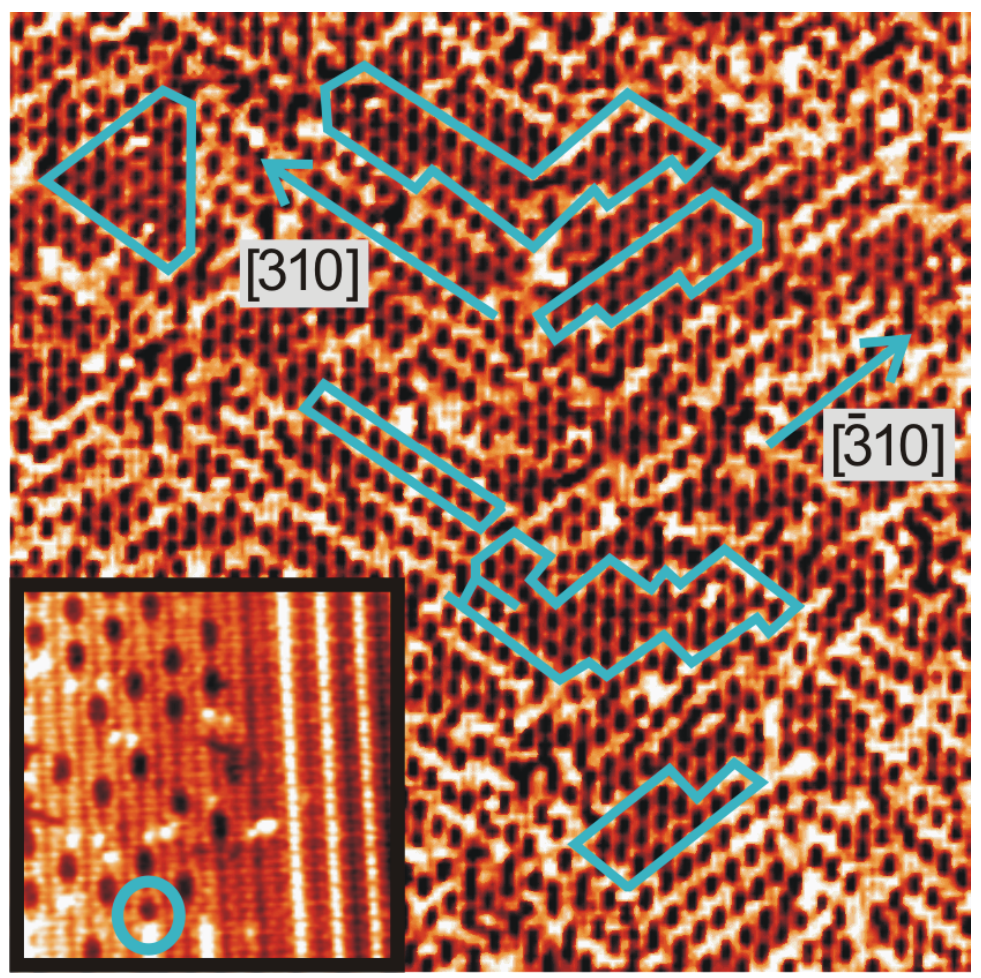

Figure 3.3: STM image of an ordered pattern of nanocavities on Pt/Ge(001) recorded at $77 \mathrm{~K}$ with a sample bias of $+1.5 \mathrm{~V}$ and tunneling current of $0.3 \mathrm{nA}$. The area of the image is $80 \times 80 \mathrm{~nm}^{2}$. Several ordered domains are outlined. The arrows indicate [310] and [ $\overline{3} 10]$ directions. The inset shows a region that contains nanocavities as well as a few nanowires (set point: $0.3 \mathrm{nA}$ and sample bias: $-1.5 \mathrm{~V}$; area: $15.8 \times 15.8 \mathrm{~nm}^{2}$ ). A nanowire that terminates near a nanocavity is indicated by a small circle.

The difference between filled and empty states STM is substantial, as shown in Fig. 3.4. For negative sample biases $(-0.8 \mathrm{~V})$ the nanocavities exhibit an elliptical shape, as shown in Fig. 3.4 (A), while at positive sample biases $(+1.0 \mathrm{~V})$, the nanocavities have a rectangular shape as depicted in Fig. 3.4(B). The longitudinal and lateral axes dimensions of the ellipses are approximately $1.6 \mathrm{~nm}$ and $0.54 \mathrm{~nm}$, respectively. However, at positive bias, the length and the width of the rectangular units are $1.6 \mathrm{~nm}$ and $0.6 \mathrm{~nm}$ respectively. We found that at low positive bias $(0.2 \mathrm{~V})$, the electronic shape transition as a function of sample bias of the nanocavities sets in.

Line scans taken across the nanocavities reveal that they are only one 

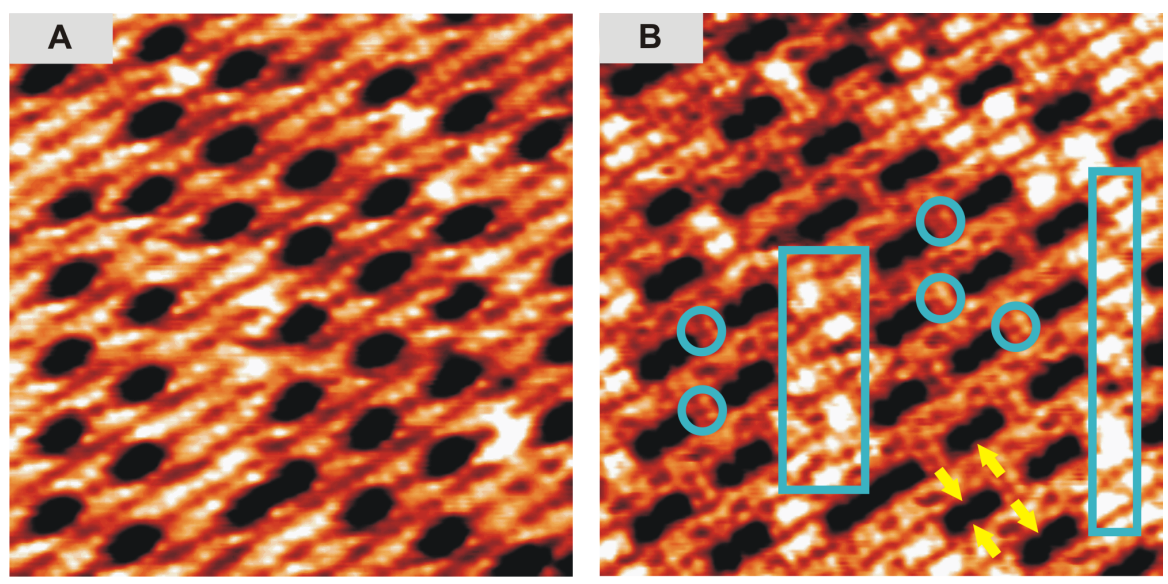

Figure 3.4: (A) Filled state (set point: $0.3 \mathrm{nA}$ and sample bias: $-0.8 \mathrm{~V}$ ) and (B) empty state images (set points: $0.3 \mathrm{nA}$ and sample bias: $+1.0 \mathrm{~V}$ ) of the nanocavities recorded at $77 \mathrm{~K}$. The image size is $13.5 \times 13.5 \mathrm{~nm}^{2}$. The regions in between the nanocavities, the anti-phase boundaries and the small protrusions at the edges of the nanocavities are outlined by small circles, large rectangles and arrows, respectively.

atomic layer deep. A careful analysis reveals the presence of two small protrusions, appearing faintly at the opposite longitudinal edges of the rectangular nanocavity. A few of these protrusions are indicated by arrows in Fig. 3.4(B). These protrusions are absent in filled state images. It is frequently observed that when two or more nanocavities are sufficiently close they merge together to form a longer cavity. But, it is very rare that nanocavities merge together along their lateral axis direction, i.e. in a direction perpendicular to substrate dimer rows.

\subsubsection{Electronic Properties}

In order to investigate the electronic density of states of the nanocavity arrays in more detail, we performed differential conductivity measurements. Figure 3.5 shows the differential conductivity $\left(\frac{d I}{d V}\right)$ curves recorded at regions between the neighboring nanocavities in the densely packed areas and at anti-phase boundaries (circles and rectangles in Fig. 3.4(B), respectively). The solid line corresponds to the differential conductivity, $\frac{d I}{d V}$, at regions between neighboring nanocavities (neck positions), whereas the dashed line corresponds to anti-phase boundaries. The curves are quite similar except for the fact that a 


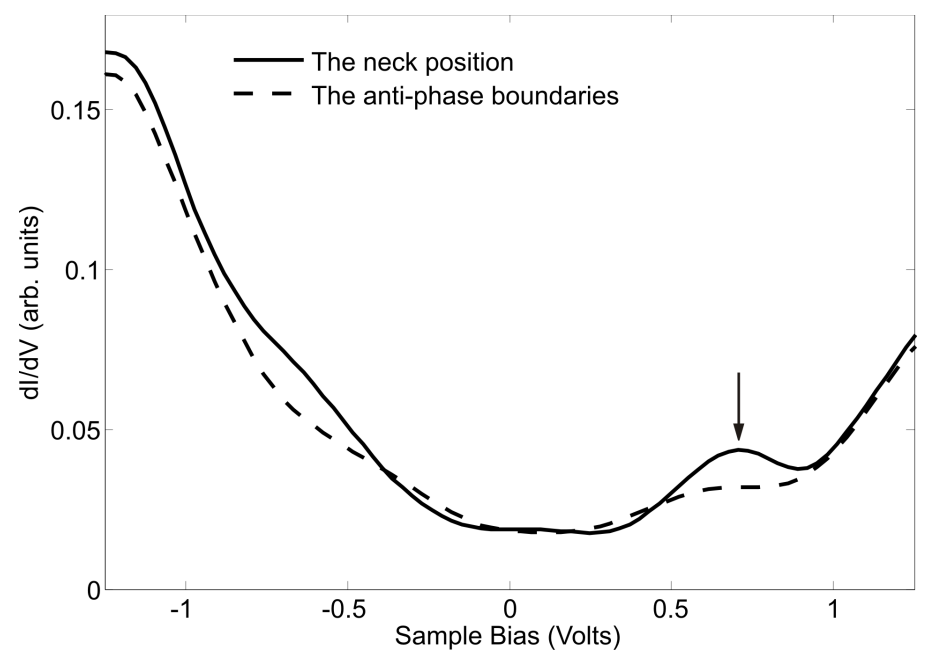

Figure 3.5: Differential conductivities $\left(\frac{d I}{d V}\right)$ recorded at $77 \mathrm{~K}$ at the regions located between neighboring nanocavities (the neck positions) in the well-ordered and densely packed arrays (solid line) and at the anti-phase boundaries (dashed line) of the ordered domain pattern (set point: $0.3 \mathrm{nA}$ and sample bias: $+0.8 \mathrm{~V}$ ). The peak located at $0.7 \mathrm{~V}$, indicated by an arrow, corresponds to an electronic state located at the regions between neighboring nanocavities in the well ordered and densely packed arrays.

strong peak at $0.7 \mathrm{~V}$ is present in the regions between neighboring nanocavities. We also recorded derivative $\left(\frac{d I}{d V}\right)$ maps to investigate the spatial dependence of this electronic state at the surface. Figure 3.6 shows (A) topography, (B) derivative map and $(\mathrm{C})$ a superimposition of derivative and topography map (set point: $0.3 \mathrm{nA}$ and sample bias: $+0.8 \mathrm{~V}$ ). The topography and derivative maps were recorded simultaneously, so that any drift between the two measurements is avoided. Figure 3.6(C) shows an ordered array of bright features (high electronic density) located at the regions between neighboring nanocavities. Other systems exhibiting spatially confined electronic states on comparable length scales are for instance graphene on ruthenium (79) and dicarbonitriles-sexiphenyl molecules on $\mathrm{Ag}$ (111) (80).

As has been pointed out in previous studies (49, 80), the Pt-nanowires reside in the troughs of the substrate dimer rows of $\mathrm{Ge}(001)$. This is also evident from the inset of Fig. 3.3 , where a nanowire terminates in the middle of a trough. These troughs are formed when two adjacent nanocavities merge together in the direction of their longitudinal axis. 

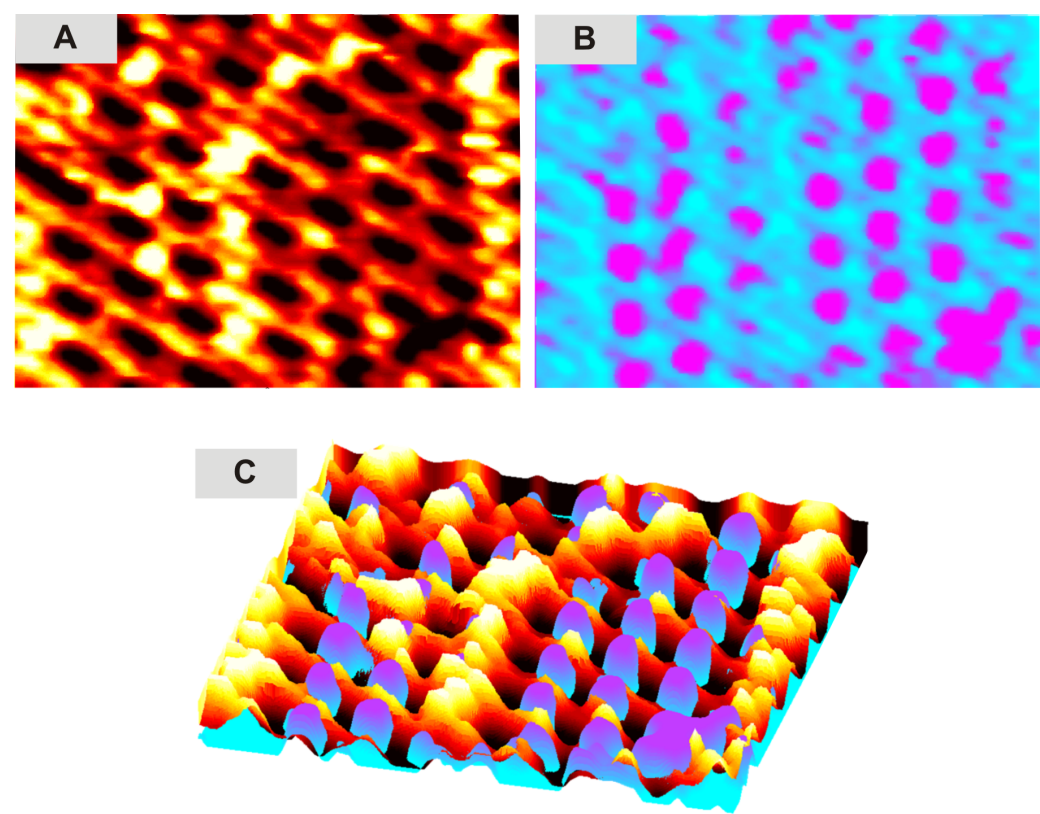

Figure 3.6: (A) Topography and (B) derivative (dI/dV) map recorded at $77 \mathrm{~K}$ (set point: $0.3 \mathrm{nA}$ and sample bias: $0.8 \mathrm{~V}$ ). (C) shows a 3-dimensional view of the superposition of the derivative map (B), onto the topography image (A). The size of each image is $16.2 \times 11.8 \mathrm{~nm}^{2}$.

\subsubsection{Structure of the Nanocavities}

The anti-phase boundaries of the ordered domains of nanocavities consist of dimer rows similar to the $\beta$-terrace as shown by the small green ellipses in Fig. 3.7. The dimer rows are comprised of Ge-Ge and Ge-Pt dimers in a 1:1 ratio (61). At first glance, the regions between and around the densely packed nanocavities seem to consist of dimer rows as well. However, Fig. 3.7 which is recorded at a sample bias of $+1.5 \mathrm{~V}$ clearly reveals that these regions consist of ordered clusters of atoms, i.e. tetramers or even larger units, rather than normal dimers. The ordered atomic clusters appear regularly at the longitudinal boundaries of the nanocavities, as shown by the large yellow ellipses. The right hand image is an enlarged zoom of the region outlined in Fig. 3.7. The ordered clusters are indicated by small bright circles. They appear to be rotated with respect to each other, similar to the tetramers of the $\mathrm{Ge} / \mathrm{Ag}(001)$ system, as reported by Oughaddou et al. (81). At the regions between neighboring nanocavities, there are also four geometrically 


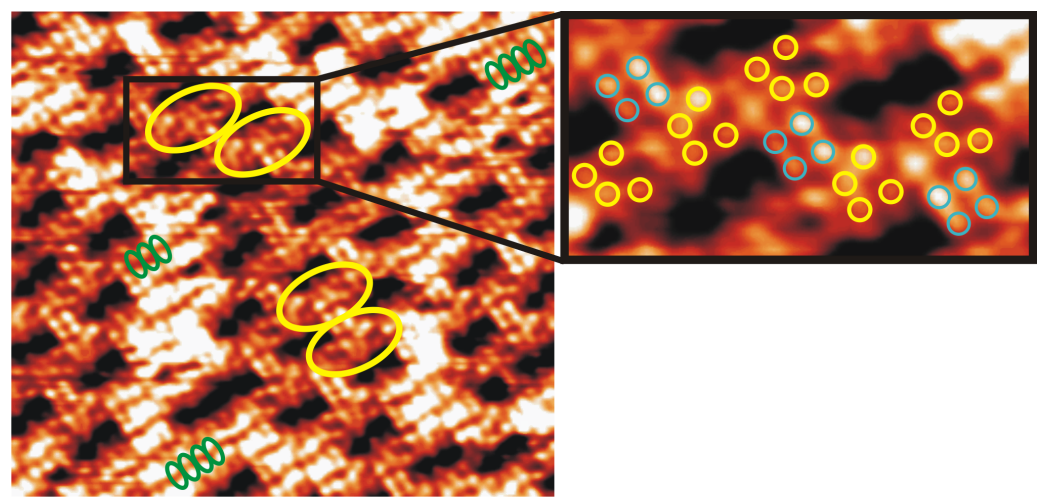

Figure 3.7: The image on the left is recorded at $77 \mathrm{~K}$ (set point: $0.3 \mathrm{nA}$ and sample bias: $+1.5 \mathrm{~V}$; area: $14.7 \times 13.2 \mathrm{~nm}^{2}$ ) and shows ordered clusters appearing regularly at the longitudinal boundaries of the nanocavities. The Pt-Ge and GeGe dimers are found at the anti-phase boundaries, which are indicated by smaller ellipses. The right image is a detailed view of the rectangular box in the left image. The two different types of atomic clusters are outlined by small circles.

symmetric bright spots that constitute another type of cluster, as outlined in the right hand image of Fig. 3.7 by small dim circles. These clusters appear to be different from the asymmetric ones in the sense that they exhibit a pronounced electronic state at $0.7 \mathrm{~V}$. Despite the high resolution STM images we are unable to put forward a structural model for the ordered nanocavity domains.

\subsection{Conclusion}

We study formation a new phase consisting of densely packed and well ordered arrays of nanocavities on a $\mathrm{Pt} / \mathrm{Ge}(001)$ surface. The nanocavities are found on the $\beta$-terrace and sometimes next to nanowires. The nanocavities appear as ellipses for negative sample biases and as rectangles for positive sample biases. An electronic shape transition of these cavities has been observed at low positive sample bias of $0.2 \mathrm{~V}$. The region between neighboring nanocavities in the densely packed arrays exhibits a well pronounced electronic state at 0.7 $\mathrm{eV}$ above Fermi energy. The well-ordered structure between the nanocavities consists of symmetric and asymmetric tetramers rather than dimers. A clear explanation of such structures would require an incorporation of more complex atomic arrangements. 


\section{Chapter 4}

\section{Transport Through a Single Octanethiol Molecule}

In this chapter, we demonstrate how an electrode-molecule-electrode junction can be controllably opened and closed by careful tuning of the contacts' interspace and voltage. The molecule, an octanethiol, flips to bridge a $\sim 1 \mathrm{~nm}$ interspace between substrate and scanning tunneling microscope (STM) tip when an electric field between them exceeds a threshold value (switch 'on'). Reducing the field below this threshold value leads to a reproducible detachment of the octanethiol molecule (switch 'off'). Once contacted, a further reduction of the contacts' interspace leads to an increase of the conductance of the molecule. Furthermore, we carried out a temperature-dependent transport study of the octanethiol molecule in this configuration. At each temperature the molecule is brought into contact by decreasing the gap between STM tip and substrate in a controlled way. The conductance of octanethiol is found to be temperature independent, indicating that either tunneling or ballistic transport is the main transport mechanism. 


\subsection{Introduction}

In the mid-1970s, Aviram and Ratner (1) put forward an elegant idea to use single molecules as functional building blocks for electronic devices, such as a transistors, diodes, memories and switches. Development and realization of such "molecular electronics" devices requires an in-depth understanding of the electronic transport properties of single molecules (82). The ability to understand, control, and exploit the transport properties of single molecules is not only of great interest from the technological point of view (3), but is also essential for further progress and expansion of science in general. For the measurement of electrical conduction through a single molecule one has to connect macroscopic electrodes to each end of the single molecule. This, at first glance, very elementary and simple task, turns out to be extremely difficult to implement (3-5). Cross-wire junctions (13), mechanically-controlled break junctions (9, 10) and scanning tunneling microscopy (break junctions) (44, 18, 20, 83, 84) are the main techniques that have been used to probe the transport properties of molecules as described in chapter 1 . In these studies the conduction often occurs through an ensemble of molecules and therefore the transport properties are averaged out. Moreover, there is also an uncertainty about how the molecule(s) are trapped between the electrodes.

To address these challenges, a novel approach was devised where a preselected molecule had been lifted from the substrate and analyzed (21-23, 25, 85) by scanning tunneling microscope (STM). Using this approach Lafferentz et al. (23) studied the transport properties of a single polyfluorene wire as a function of length, whereas Temirov et al. (25) addressed the Kondo effect of perylene-tetracarboxylic-dianhydride molecules. More recently, similar methods were applied by Leary et al. (22) to study the transport through bifluorene molecules that were capped with $\mathrm{C}_{60}$ fullerenes whereas Toher et al. studied the electrical transport of perylene-tetracarboxylic-dianhydride molecules (85). In 2009, Kockmann et al. (21) also showed that a single octanethiol $\left(\mathrm{CH}_{3}\left(\mathrm{CH}_{2}\right)_{7} \mathrm{SH}\right)$ molecule can be trapped between a Pt nanowire and the apex of an STM tip. At a current set point of $1 \mathrm{nA}$ and sample bias of $+1.5 \mathrm{~V}$ the octanethiol molecule occasionally jumps up to bridge STM tip and substrate and the charge transport occurs through the molecule. This method allowed the measurement of the conductance of a single, pre-selected octanethiol molecule. However, control over the attachment and detachment 
process of the octanethiol molecule was not obtained.

There are several possible molecular transport mechanisms applicable to single molecular junctions, e.g., thermionic emission, hopping, Fowler-Nordheim tunneling, ballistic and direct (coherent) tunneling (5). Thermionic emission and hopping are temperature dependent, whereas direct tunneling, FowlerNordheim and ballistic transport are temperature independent phenomena. Direct tunneling usually occurs at biases smaller than the work function, while Fowler-Nordheim tunneling takes place at biases that exceed the work function. In addition, in Fowler-Nordheim tunneling the STM setup will lead to field emission resonances. They are referred to as Gundlach oscillations, which show up as well-defined steps in current-voltage (I-V) and distance-voltage $(\mathrm{z}-\mathrm{V})$ traces $(86,87)$. To understand the transport mechanism through octanethiol molecules a temperature dependent measurement would be needed. Temperature dependent transport measurement are very challenging for STM molecular junctions as even the slightest change in temperature will lead to a change of the substrate-tip separation and thus to a compressed, stretched, or detached state of the molecule. This is presumably the main reason why there are only few examples in literature where single molecule conductance has been measured as a function of temperature. An exception is a recent study by Sedghi et al. (88) where the temperature dependence of the conductance of short chains of porphyrin molecules was measured in a temperature range from 300 to $375 \mathrm{~K}$.

In this chapter, we show controlled transport through a single octanethiol molecule trapped between an STM tip and Pt/Ge(001) substrate followed by variable temperature measurements of this molecular junction. Full control over the jump into and out of contact of the molecule has been obtained by carefully adjusting the distance between the STM tip and the substrate. This tip-molecule-substrate junction acts as a molecular switch that can be opened and closed by varying the voltage across the junction. A careful analysis reveals that the jump into and out of contact is governed by the electric field between both electrodes. The threshold electric field for attachment/detachment is $4-6 \times 10^{9} \mathrm{Vm}^{-1}$. In addition, we show that further reduction of the contacts' interspace leads to an increase of the conductance of the molecule. The controllability of this junction, i.e., the ability to manage the cessation and initiation of contact with high accuracy, allows for a feedback mechanism that can be sustained over a wide temperature range. Using this recipe, we perform variable 
temperature transport measurements through a single octanethiol molecule. This approach differs significantly from methods that use static contacts for the molecule, e.g., break junction methods $(10,89,90)$. In our work, at every measurement the contact was freshly established, and after the current-distance (I-z) measurements were recorded the contact was broken again, so that the junction could rearrange itself to its reference point. Therefore, the junction itself remained uniform, i.e., in particular the electrode-electrode separation, throughout the whole experiment.

\subsection{Experimentation}

We have used $\mathrm{Pt} / \mathrm{Ge}(001)$ substrates (61, 91), as described in the chapter 3 , to immobilize the octanethiol molecules and the Omicron low temperature scanning tunneling microscope (LT-STM) for transport measurements. The flat $\mathrm{Pt} / \mathrm{Ge}(001)$ substrates $\left(4 \times 10 \mathrm{~mm}^{2}\right)$ were prepared and exposed to octanethiol molecules in the preparation chamber. Cleaning of Ge(001) substrate was done by cycles of ion bombardment using $500 \mathrm{eV} \mathrm{Ar}{ }^{+}$ions followed by annealing at $1100 \mathrm{~K}$. This process was repeated several times until atomically clean $\mathrm{Ge}(001)$ surfaces were obtained (92). Subsequently, we deposited 0.5 monolayers of $\mathrm{Pt}$ onto the clean $\mathrm{Ge}(001)$ surface at room temperature followed by annealing at $1100 \mathrm{~K}$. This leads to the formation of regions covered with atomic Pt chains. The majority of these chains exhibit a mutual spacing of $1.6 \mathrm{~nm}$. More detailed information on the procedure, the formation and the properties of these self-organizing atomic Pt chains is given in (49, 61, 91). The Pt/Ge(001) substrate was subsequently exposed to octanethiol molecules (98.5\% pure, purchased from Sigma-Aldrich) via a leak valve, with a precise control of exposure. We exposed the substrates to a pressure of $2.5 \times 10^{-7}$ Torr for 40 seconds leading to an exposure of $\sim 10$ Langmuir (L) of octanethiols. The substrate was then transferred to the STM chamber and cooled down to $77 \mathrm{~K}$ to carry out the measurements.

For the temperature dependent measurement, the cryostat of the LT-STM was left to heat up slowly once the liquid nitrogen was almost fully evaporated. This warming up process is slow and stable enough to carry out the measurements at a fixed temperature for a while. As the temperature was carefully monitored, a series of I-z curves were recorded after every few degrees of increment (typically $1-2 \mathrm{~K}$ ) from 77 to $172 \mathrm{~K}$. Beyond $180 \mathrm{~K}$ the 
thermal drift prohibited us to achieve accurate and well reproducible measurements. All the traces were recorded at a positive bias voltage of $+1.5 \mathrm{~V}$ and a setpoint current of $0.5 \mathrm{nA}$. After each I-z measurement the feedback loop was switched back on again to allow the tip to move back to its initial position, thus breaking the contact again. At this point the junction returns to the original configuration defined by the setpoint value, and thereby providing us with a well-defined and fixed reference point. The $z$-scale for all the I-z measurements is corrected to compensate for the temperature-dependent response of the piezo tubes by applying a factor of the expansion coefficient.

\subsection{Results and Discussion}

\subsubsection{Transport at $77 \mathrm{~K}$}

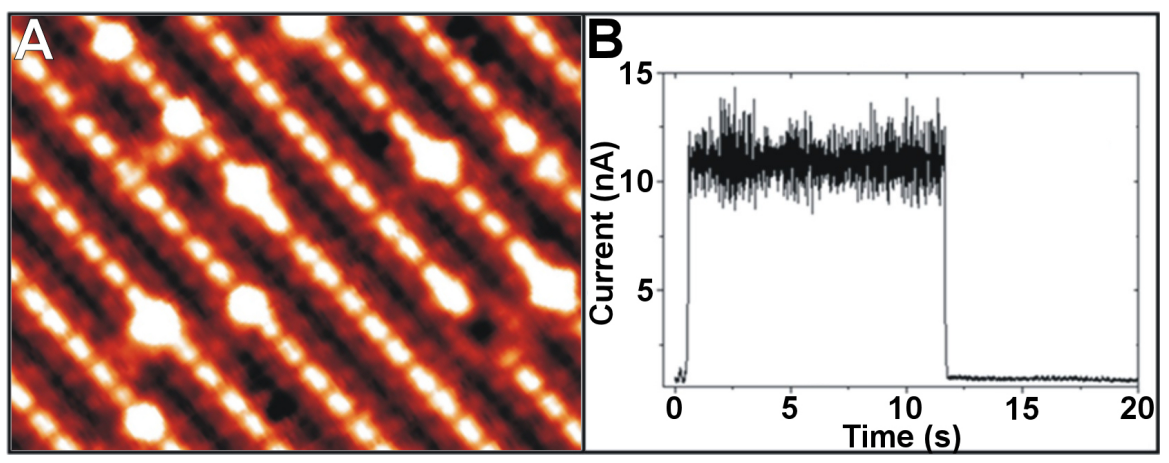

Figure 4.1: (A) Filled state STM image recorded at $77 \mathrm{~K}$ of octanethiol molecules immobilized on a $\mathrm{Pt} / \mathrm{Ge}(001)$ surface (setpoint: $0.5 \mathrm{nA}$ and sample bias: $-0.9 \mathrm{~V}$ ). The large, bright features are octanethiol molecules adsorbed on the Pt nanowires. (B) A current-time trace (I-t) shows a jump in the current to $\sim 11 \mathrm{nA}$ as the molecule makes contact to the tip (adapted from (21)).

A filled state STM image of a Pt/Ge(001) substrate, exposed to $10 \mathrm{~L}$ of octanethiols $\left(\mathrm{CH}_{3}\left(\mathrm{CH}_{2}\right)_{7} \mathrm{SH}\right)$, recorded at $77 \mathrm{~K}$ is shown in Fig. 4.1(A). Open loop current-time measurements performed on top of an octanethiol molecule showed that the gap between tip and substrate can be closed by the molecule making contact with the tip. During acquisition of the current-time traces the tail ( $\mathrm{C}-\mathrm{H}$ end) of the molecule flipped into contact with the tip, resulting in an increase of the current, jumping from its setpoint value $(1 \mathrm{nA})$ to $11-$ $15 \mathrm{nA}$ as shown in Fig. 4.1(B). See ref. (21) for details. The tail typically 
remained in contact for a few tens of seconds before it detached again. As the length of the octanethiol molecule $(\sim 1 \mathrm{~nm})$ nicely fits into the vacuum gap between the substrate and the STM tip, the extracted resistance value of $100-140 \mathrm{M} \Omega$ corresponds to the resistance of a single octanethiol molecule at $+1.5 \mathrm{~V}$, which is in good agreement with values reported in the literature (92 94). It should be noted that the molecule jumped randomly in and out of contact and therefore it was not possible to obtain full control over the switching process.

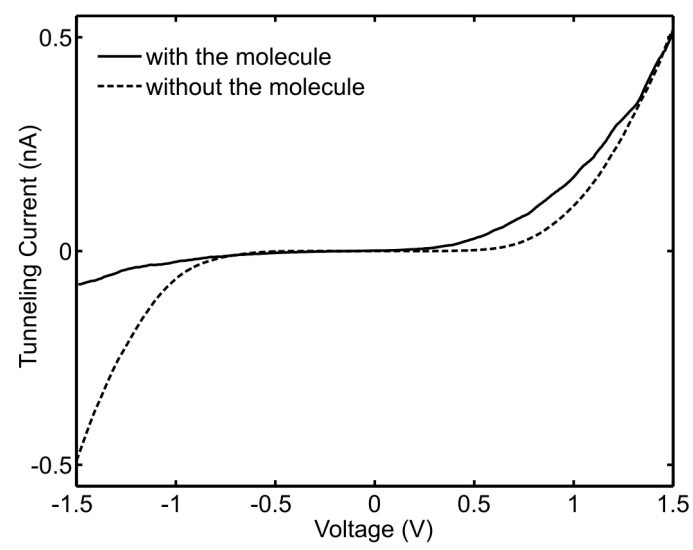

Figure 4.2: Tunneling current-voltage (I-V) curves of the tunnel junctions recorded at $77 \mathrm{~K}$ before and after the STM tip has picked up an octanethiol molecule. For both traces we have used a setpoint value of $0.5 \mathrm{nA}$ at $1.5 \mathrm{~V}$.

In this work, we are able to attach the head (S-H end) of a single octanethiol molecule to the tip by recording current-time traces at tip-surface distances smaller than $1 \mathrm{~nm}$. When the sulfur atom of the octanethiol makes contact with the tungsten STM tip, it forms a strong bond and therefore the tail of the octanethiol is usually fully released from the surface upon retraction of the tip. Current-voltage (I-V) curves of the tunnel junctions recorded using a tip decorated with an octanethiol molecule are significantly different from I-V curves recorded using a tip without a molecule (see Fig. 4.2. Both $\mathrm{I}-\mathrm{V}$ curves are recorded on the $\mathrm{Pt}$ nanowires at a setpoint of $0.5 \mathrm{nA}$ and $+1.5 \mathrm{~V}$ bias voltage. A molecule being attached to the STM tip leads to rectifying characteristics of the I-V traces of the junction. This behaviour can be attributed to the large band gap of alkanethiol molecules with the highest occupied molecular orbital (HOMO) being close to the Fermi level. In the 
case of alkanethiol molecules on $\mathrm{Au}$, the HOMO lies $2 \mathrm{eV}$ below the Fermi energy (95). For a positive substrate bias, the electrons tunnel from the tip through the molecule to the surface giving rise to higher current compared to a negatively biased substrate.

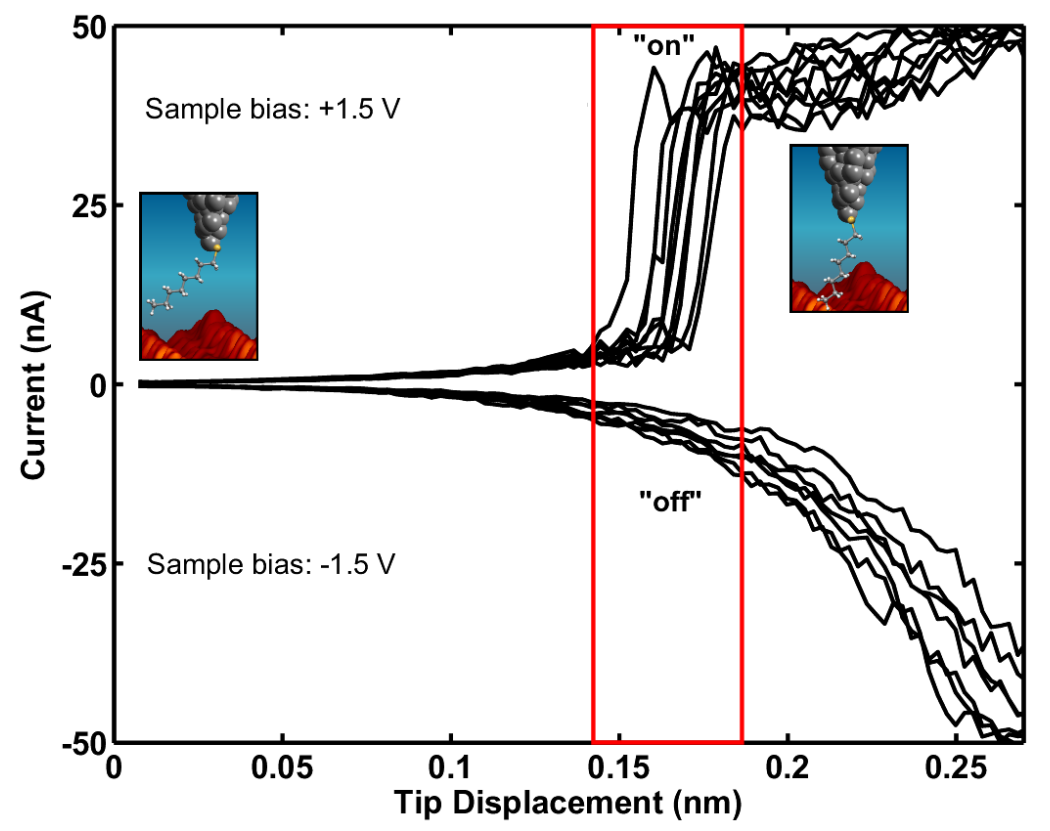

Figure 4.3: Current vs tip-displacement curves recorded with an octanethiol molecule attached to the apex of the STM tip. Top: the sample bias is $+1.5 \mathrm{~V}$ and the setpoint current is $0.2 \mathrm{nA}$. As the STM tip approaches the substrate by $\sim 0.15 \mathrm{~nm}$ the molecule makes contact and the current jumps to 35-40 nA ('on' state). Bottom: the sample bias is $-1.5 \mathrm{~V}$ and the setpoint current is $0.2 \mathrm{nA}$. The octanethiol molecule does not jump into contact ('off' state).

We performed a series of current-distance (I-z) measurements with an octanethiol molecule attached to the tip, at various locations on the sample surface. The sample bias was set to $+1.5 \mathrm{~V}$ and the tunneling current to 0.2 nA (see Fig. 4.3). After bringing the STM tip closer to the substrate by a distance $\Delta z=0.15-0.18 \mathrm{~nm}(\Delta z$ refers to the $z$-displacement of the tip towards the surface with respect to the set point height), the octanethiol molecule makes contact with the substrate and the current jumps to higher values of 35 $\pm 5 \mathrm{nA}$ (see Fig. 4.3). The fluctuation in the current may be attributed to the various contact geometries the molecule can have with the STM tip and the substrate. For a negative sample bias, however, the octanethiol never jumps 
into contact and the tunneling current shows an exponential dependence with distance. The position of the transition from 'off' to 'on' (see the box in Fig. 4.3 depends on the actual value of the applied bias voltage. The I-V and I-z spectroscopy data provide strong evidence for a successful attachment of a single octanethiol molecule to the apex of the tip.

By using the current decay constants for electron transport through a vacuum gap $\left(22.0 \mathrm{~nm}^{-1}\right.$; see ref. (5)) and an alkanethiol molecule $\left(8.1 \mathrm{~nm}^{-1}\right.$; see ref. (5)), we can estimate the separation between the freely hanging end of the octanethiol molecule ( $\mathrm{C}-\mathrm{H}$ end) and the substrate using Simmons's model (39, 96). The conductance, $G_{\text {contact }}$, corresponds to the case where the molecule makes contact with the substrate and the transport occurs through the molecule, while $G_{\text {non-contact }}$ refers to the case where the molecule is out of contact and the transport occurs through the molecule and vacuum gap. $G_{\text {contact }}$ should also include the contact conductance between molecule and substrate. However, this contact resistance is much smaller than the resistance of a single octanethiol molecule and therefore it can safely be ignored. The conductance ratio, i.e. $\frac{G_{\text {contact }}}{G_{\text {non-contact }}}\left(\frac{G_{\text {contact }}}{G_{\text {non-contact }}} \approx \frac{23 n A}{0.16 n A} \approx 145\right)$, can be extracted from Fig. 4.3 . If $L(\sim 1 \mathrm{~nm})$ is the length of the molecule, $G_{\text {contact }}$ will be proportional to $e^{-8.1 L}\left(\right.$ (5). We can calculate $G_{\text {non-contact }}$ from the total resistance when the transport occurs through the molecule and the vacuum gap. The total conductance can in this case (coherent tunneling) be factorized (5), i.e.

$$
\frac{G_{\text {contact }}}{G_{\text {non-contact }}} \approx \frac{e^{-8.1 L}}{e^{-8.1 L} e^{-22 d}}=e^{22 d} \approx 145,
$$

where $d$ is the vacuum gap between the freely hanging end of the molecule and the substrate when the tip is positioned at its setpoint height. From equation 4.1 we find that $d$ is about $0.23 \mathrm{~nm}$.

It is important to note that the separation, $d$, is significantly larger than the tip displacement required for the molecule to make contact. Therefore, it is very likely that the flexible tail of the molecule jumps into contact beyond a certain threshold value of the electric field. Before jumping into contact the tail of the molecule is at an angle with respect to the shortest tip/substrate trajectory, as shown in Fig. 4.3. This angle tends to decrease upon changes in the electric field between tip and substrate as the tip approaches the surface. It is also evident from these traces that only one molecule is attached to the tip. For the attachment of more than one molecule, one would see multiple jumps in the current. The current in the contact is about $\sim 2.5$ times larger than 
reported for the case where the sulfur atom of the octanethiol is attached to the Pt nanowire (21). There can be several reasons for this factor of 2.5. One viable explanation for this difference is that the number of $\mathrm{C}$ atoms trapped in the junction is now seven rather than eight as in the case of Kockmaan et al. (21). The conductance $G$ of an alkanethiol molecule decreases exponentially with the length of the molecule, i.e. $G \propto e^{-\beta L}$. The value of the decay constant $\beta$ is $8.1 \mathrm{~nm}^{-1}$. If one carbon atom less is involved in the transport, the effective length of the molecule is reduced and the conductance will increase by a factor 2.5 1 This is in very good agreement with our observations. Other possibilities may include a different adsorption geometry of the molecule at the apex of the tip as compared to the situation in Fig. 4.1(21), e.g. sliding of the molecule along the electrode, a different electrode material ( $\mathrm{Pt}$ or $\mathrm{W}$ ) or a deformation of the electrode. At this stage, it is difficult to determine the exact adsorption geometry of the molecule at the apex of the STM tip or the exact morphology of the electrodes.

In Fig. 4.4 a set of three consecutively recorded current-voltage traces are shown. For each measurement the voltage is repeatedly ramped (ten times) from $+1.5 \mathrm{~V}$ to $-1.5 \mathrm{~V}$ as shown in the bottom section. During the whole measurement the feedback loop of the STM is disabled (97). In addition, after each I-V trace, the STM tip is moved $0.05 \mathrm{~nm}$ closer or away from the substrate as shown in the top section. The I-V traces are shown in the middle section. For I-V traces numbered 1-6, the tip was moved closer to the surface and similarly for I-V traces numbered 7-10 the tip was moved away from the surface in steps of $0.05 \mathrm{~nm}$ before the measurement. After approaching the substrate by $0.10-0.15 \mathrm{~nm}$ at $+1.5 \mathrm{~V}$ the octanethiol molecule immediately jumps into contact ('on' state). However, at negative sample bias the molecule remains always in its 'off' state. The contact at positive bias breaks again as soon as the voltage is reduced below a threshold value of about $0.4 \mathrm{~V}$. This threshold voltage changes with changing gap spacing indicating that the attachment/detachment is governed by the electrical field, rather than the voltage, as shown in Fig. 4.5. The molecule jumps into (out of) contact for an electric field higher (lower) than threshold value of $4-6 \times 10^{9} \mathrm{Vm}^{-1}$. This is also consistent with the I-z measurements recorded at various substrate bias values. We found that for smaller sample biases attachment occurs at

\footnotetext{
${ }^{1}$ The projected $\mathrm{C}-\mathrm{C}$ bond length along the long axis of the octanethiol molecule is 0.125 nm.
} 


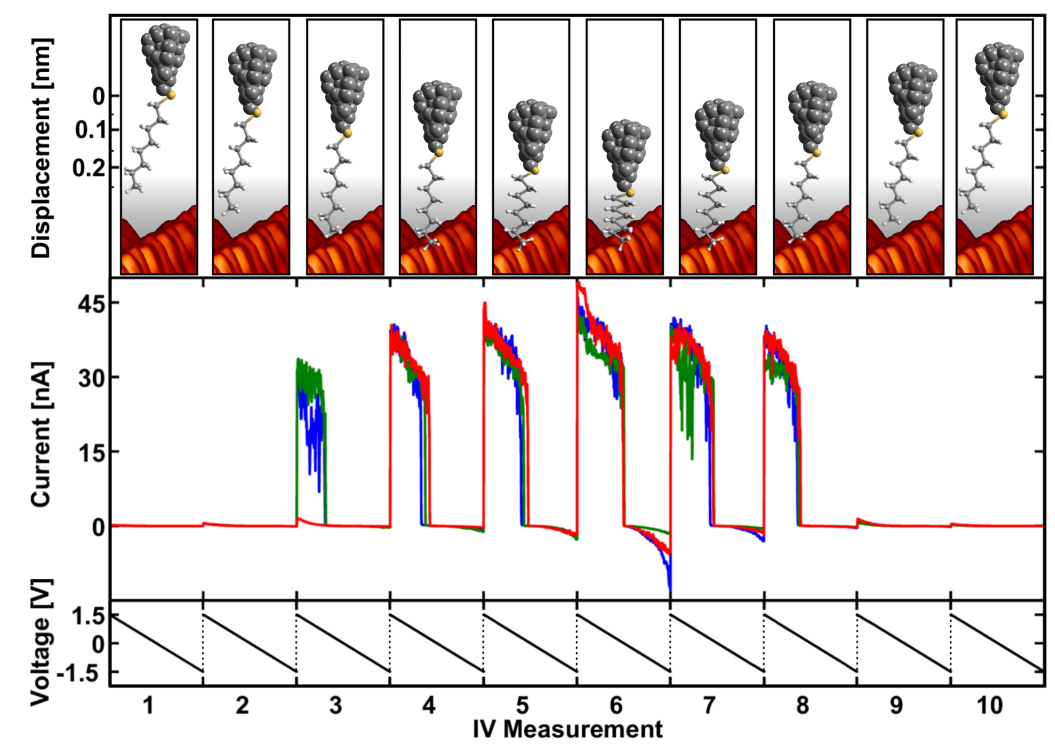

Figure 4.4: A set of three I-V curves (middle section; red, blue and green curves) recorded in series, with varying tip-substrate distance and the feedback loop disabled. The top section shows a simplified cartoon of the molecule attached to the apex of the STM tip and its relative position with respect to the substrate. The bottom section shows a series of bias voltage ramping from +1.5 to $-1.5 \mathrm{~V}$. The $\mathrm{I}-\mathrm{V}$ measurements are acquired after the tip has moved (in steps of $0.05 \mathrm{~nm}$ ) and stabilized. Traces 1-6 correspond to the tip's relative position from the setpoint height (i.e. $0.2 \mathrm{nA}$ and $1.5 \mathrm{~V}$ ) to $0.25 \mathrm{~nm}$, while traces $7-10$ correspond to the relative position from 0.20 to $0.05 \mathrm{~nm}$. After approaching the substrate by $0.1-0.15$ $\mathrm{nm}$ (traces 3-4) the octanethiol molecule jumps into contact and for I-V trace 8 the molecule jumps out of contact.

slightly larger $\Delta z$. However, it should be noted that this controlled switching only occurs if the STM tip is sufficiently close to the surface (i.e. 0.7-0.8 $\mathrm{nm})$. Traces 3 and 8 are borderline cases $(\Delta z=0.1 \mathrm{~nm})$ since the jump into and out of contact is not fully under control yet (see I-V measurement 3 ). We believe that the latter is related to the length of the octanethiol molecule. The octanethiol molecule has a length of about $1 \mathrm{~nm}$ and thus nicely fits in the tunnel junction for a tunneling current of $0.2-1.0 \mathrm{nA}$, however in this case the bonding is rather weak since only one $\mathrm{C}$ atom can interact with the electrode. For a substrate-tip separation of less than $1 \mathrm{~nm}$ (after a tip displacement of $0.15 \mathrm{~nm}$ with respect to the setpoint height) the bonding becomes significantly stronger due to the possibility of more $\mathrm{C}$ atoms interacting with the electrode 
(see top panel of Fig. 4.4). This interpretation is strongly supported by the observation that the conductance of the trapped octanethiol molecule is larger by a factor of 2.5 as compared to the case where the tip-substrate separation is at its initial distance, i.e. $\Delta z=0 \mathrm{~nm}$.

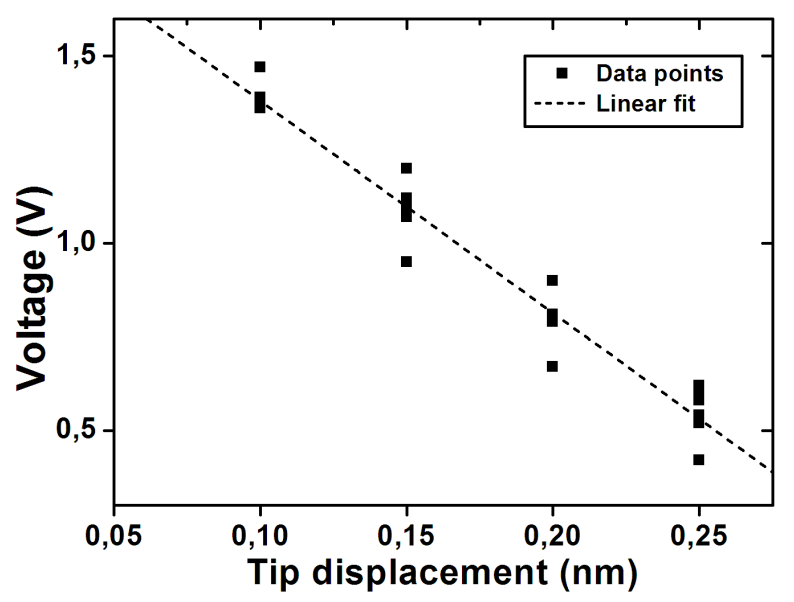

Figure 4.5: Threshold voltage is plotted against tip displacements while the tip moves towards the surface. We extract a threshold electric field value from the slope of the linear fit to be $4-6 \times 10^{9} \mathrm{Vm}^{-1}$.

Upon a further reduction of the contacts' interspace the conductance of the octanethiol molecule gradually increases. From Figs. 4.3 and 4.4 we extract that the conductance increases roughly by $20 \%$ per $0.1 \mathrm{~nm}$ of compression. This value is substantially smaller than the increase of about $200 \%$ as predicted by the exponential dependence of the conductance, on the length of the molecule (see footnote 1 ). The $\mathrm{C}-\mathrm{C}$ bonds are arranged in a zigzag structure, with a bond length of $0.154 \mathrm{~nm}$ and a $\mathrm{C}-\mathrm{C}-\mathrm{C}$ bond angle of $109^{\circ}$. It is likely that the bond angles become slightly smaller upon compression with the C-C bond length remaining unaltered. In order to explain our experimental data we have to assume that either the change in $\mathrm{C}-\mathrm{C}-\mathrm{C}$ bond angle significantly reduces the decay constant or that the molecule is bent within the tunnel junction or it is sliding along the contact.

Figure 4.5 shows voltage dependence of tip-displacement as the molecule jumps into contact. A threshold value of electric field, $E$, corresponding to molecule jumping into contact is extracted from the slope of the linear fit which equals to $4-6 \times 10^{9} \mathrm{Vm}^{-1}$. The molecule jumps into (out of) contact for an electric field higher (lower) than this threshold value $E$. An initial tip- 
substrate distance, $z_{0}$ can be calculated from the linear fit using equation

$z_{0}=\frac{V_{\Delta z=0}}{E}$. Using $V_{\Delta z=0} \approx 1.9 \mathrm{~V}$ and $\mathrm{E}$ is $4-6 \times 10^{9} \mathrm{Vm}^{-1}, z_{0}$ comes out to be $\sim 0.48 \mathrm{~nm}$, which deviates from $1 \mathrm{~nm}$ of assumed tip-substrate distance. A linear extrapolation may deviate for very smaller and larger tip-displacements which may be the reason that value of $z_{0}$ deviates from $1 \mathrm{~nm}$ of tip-substrate distance.

\subsubsection{Temperature Dependent Transport}

We performed temperature dependent measurements using the above configuration of tip-molecule-substrate junction to study electronic transport mechanism in an octanethiol molecule. I-z measurements at different temperatures were carried out as described in the experimental section of this chapter. Figure 4.6. A) shows current-tip displacement (I-z) measurements recorded at temperatures from $77 \mathrm{~K}$ to $172 \mathrm{~K}$. All the I-z curves are corrected to compensate for thermal expansion of the piezo using a factor of the expansion coefficient.

As we see in Fig. 4.6(A), the temperature dependent measurements are very comparable to the results obtained at $77 \mathrm{~K}$ corresponding to switching behavior of a single octanethiol molecule as described previously. First, the jump into contact occurs at the same tip-substrate separation $(\Delta z=0.15-0.18$ $\mathrm{nm}$ with respect to height corresponding to sample bias of $1.5 \mathrm{~V}$ and setpoint current $0.5 \mathrm{nA}$ ). This variation in $\Delta z$ is comparable to the measurements that were recorded at $77 \mathrm{~K}$. Second, the current value that is reached after the molecule makes contact is similar to values recorded at $77 \mathrm{~K}$, i.e., $\sim 40 \mathrm{nA}$.

Figure 4.6.(B) shows tip displacements at which the molecule jumps into contact along with the conductance of molecule plotted against temperature. Point of contact is estimated from the tip displacements $(\Delta z)$ required for the molecule to jump into contact as the current experiences a sharp increase. We extract $\Delta z$ values at $20 \mathrm{nA}$ of current through the junction as shown by a horizontal bar in the Fig. 4.6(A). The conductance values are extracted from currents measured at a tip displacement of $0.23 \mathrm{~nm}$ as shown by a vertical bar in the Fig. 4.6(A). One can easily see that within the recorded temperature window the point of contact remains amazingly constant. The mechanism for the molecule to jump into contact is thus temperature independent. This definitely supports the idea that the cause for the jump into contact is the electric field applied between STM tip and substrate, as has been put forward 

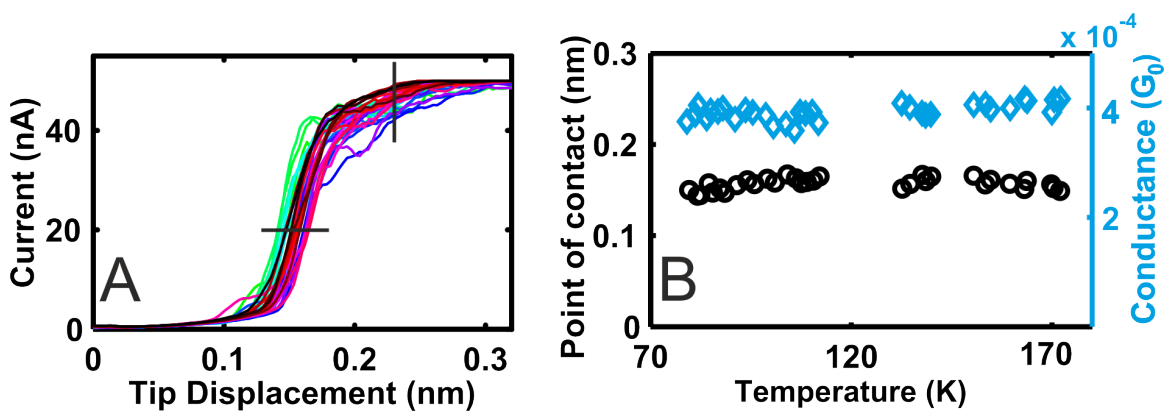

Figure 4.6: (A) Current-Tip Displacement (I-z) curves recorded with an octanethiol molecule attached to the tip at temperatures ranging from $80 \mathrm{~K}$ to 172 K. (B) Temperature dependence of point of contact $(\Delta z)$ and conductance values extracted from the the I-z curves shown in Fig. (A).

previously. Also, the thermal expansion of the molecule is too small (98) to be neglected in this temperature window. The conductance of the molecule has been determined at a sample bias of $+1.5 \mathrm{~V}$, i.e., well below the reported tunneling barrier of an octanethiol molecule (see Fig. 4.6(B)) (92). Throughout the experiment the conductance through the molecule remains constant at a value of $30 \mathrm{nS}\left(4 \times 10^{-4} G_{0}\right.$; here $G_{0}$ is quantum conductance, $\left.\frac{2 e^{2}}{h}\right)$, a value that is consistent with reports from literature (92).

In theory, the transport mechanisms that are temperature independent are ballistic transport and quantum-mechanical tunneling. In principle there are two tunneling processes: direct tunneling or Fowler-Nordheim tunneling (5, 99). Fowler-Nordheim tunneling only occurs at high voltages, i.e., voltages larger than the work function $(V>\Phi / e)$. Since the voltages applied in our experiments are significantly smaller than the $4 \mathrm{eV}$ barrier (assuming that the Fermi edge lies somewhere in the middle of the $8.9 \mathrm{eV}$ gap between highest occupied and lowest unoccupied molecular orbitals of the octanethiol molecule (100)), we have to exclude Fowler-Nordheim tunneling. The conductance of a tunnel barrier decreases exponentially with the width $(L)$ of the barrier, i.e., $G \propto \mathrm{e}^{-\beta L}$. The total conductance of the tip-molecule-substrate junction can be written as

$$
G_{\text {total }}=\frac{2 e^{2}}{h} T_{\text {total }},
$$

where $T_{\text {total }}$ is the total transmission coefficient of the tip-molecule-substrate junction. If we assume that the conductance of the octanethiol molecule is 
much smaller than the contact conductance we find that the conductance of a single octanethiol molecule is $\approx 30 \mathrm{nS}$, which agrees well with large area junctions data (100). However, hybridization of the electronic states of the molecule and the electrodes will also affect the transport mechanism. From,

equation 4.2 . $T_{\text {total }}$ can be extracted to be $\sim 4 \times 10^{-4}$ which is small enough to be in agreement with tunneling through single octanethiol molecule.

\subsection{Conclusion}

In conclusion, we have shown controlled electronic transport through a single octanethiol molecule trapped between an STM tip and a Pt/Ge(001) substrate. The molecule can be controllably attached and detached to the substrate by adjusting the electric field between tip and substrate. As the electric field exceeds the threshold value of 4-6 x $10^{9} \mathrm{Vm}^{-1}$, the molecules flips to bridge the gap between the substrate and tip which is termed as switched 'on' configuration. Reducing the field below this threshold value leads to the detachment of the octanethiol which is termed as switch 'off' configuration as the current value is substantially lower in the latter case. Once contacted, a further reduction of the contacts' inter-space leads to an increase of the conductance of the molecule. By making use of the ability of the junction to be controlled by the applied electric field, we were able to extract the transport properties of this molecule as a function of temperature. The conductance of an octanethiol molecule is temperature independent, demonstrating that the main transport mechanism is dominated by either direct quantum-mechanical tunneling or ballistic transport. The amount of control over a single molecule junction will open yet another door into the understanding and realization of molecular electronics. 


\section{Chapter 5}

\section{Electronic Properties of a $\mathrm{CuPc}$ Molecule in a "Molecular Bridge" Configuration}

In this chapter we study decoupling of the copper core in a copper phthalocyanine $(\mathrm{CuPc})$ molecule from its environment. This is realized by trapping a $\mathrm{CuPc}$ molecule between two adjacent $\mathrm{Au} / \mathrm{Ge}(001)$ nanowires in a stable "molecular bridge" configuration. We have used low-temperature scanning tunneling microscopy and spectroscopy to study the structural and electronic properties of $\mathrm{CuPc}$ in a molecular bridge configuration at $77 \mathrm{~K}$. Constant current and differential conductivity maps reveal the spatial variation of the electronic structure of the CuPc molecule. The core of the CuPc molecule in a molecular bridge configuration is dim at low sample biases, but becomes suddenly bright at a bias of $5 \mathrm{~V}$. While most of the molecules are very stable, a few diffuse laterally across the surface induced by tunneling electrons. Time resolved current spectroscopy recorded on lobes with speckles show lateral dynamics and resemble telegraphic noise. 


\subsection{Introduction}

A prerequisite to exploit molecular electronics is a firm knowledge of the properties of individual molecules. The majority of molecular electronic studies performed so far have been focused on this important aspect. It is of common knowledge that the adsorption of an atom or a molecule on a surface inevitably leads to a change of the structural and electronic properties of the atom or the molecule. Therefore, to achieve a proper contact to the molecule with the outside macroscopic world, while leaving the essential single molecular properties intact becomes a challenging task. A way out of this problem is to use more sophisticated molecules that contain end groups that can be connected to the contact pads and a shielded inner part which can act as an active element. The simplest examples of molecules that satisfy these criteria are metal-phthalocyanines (MPc).

MPc molecules are composed of a metal atom surrounded by a ligand ring. They are of technological relevance since these molecules are used in organic solar cells (101), organic field-effect transistors (102), and organic light emitting diodes (103). Self-organized mono- and multilayers of MPc molecules can be grown on various surfaces in a highly ordered fashion, making them attractive candidates for technological applications (104, 105). As they also carry non-zero electron spin, various spin based studies are also performed on such MPc molecules (106 108). In this chapter, we have investigated the $\mathrm{CuPc}$ molecule which consists of a four-lobed Pc ring with a copper ion in the centre of the molecule as shown in the inset of Fig. 5.1. The size of the $\mathrm{CuPc}$ molecule is about $1.4 \mathrm{~nm} \times 1.4 \mathrm{~nm}$. The Cu core, with oxidation state $2+$, is bound to the Pc ring with two covalent and two coordinated covalent bonds. The $\mathrm{Cu}$ ion is small enough to fit into the cavity of the Pc macrocycle, making $\mathrm{CuPc}$ a planar molecule.

The Ho group (109, 110) investigated a single CuPc molecule adsorbed on an ultrathin $\mathrm{Al}_{2} \mathrm{O}_{3}$ film grown on a $\mathrm{NiAl}(110)$ substrate. Several adsorption configurations were identified which resulted in distinctly different differential conductivity spectra. However, all spectra have the highest occupied molecular orbital (HOMO) located in the range from $0.6 \mathrm{eV}$ to $1.1 \mathrm{eV}$ below the Fermi level and the lowest unoccupied molecular orbital (LUMO) located in the range from $0.6 \mathrm{eV}$ to $0.9 \mathrm{eV}$ above the Fermi level. The LUMO state of the intact $\mathrm{CuPc}$ molecule is contributed by two orbitals $(111,112)$. The $e_{g}$ orbital is 
mainly derived from the $\pi$ states of the Pc ring and delocalized over the ring. This orbital is doubly degenerate (each located at the opposite lobes). In addition, the LUMO state is contributed by the $b_{1 g}$ orbital that is dominated by the $\mathrm{Cu} 3 d$ states (mainly by the $3 d_{x^{2}-y^{2}}$ state lying in the plane of the molecule) and localized on the $\mathrm{Cu}$ core. The HOMO is related to the $a_{1 u}$ orbital derived from the $\pi$ states of the Pc ring. Similar to the $e_{g}$ orbital, the $a_{1 u}$ orbital is delocalized over the $\mathrm{Pc}$ ring, but this state is not degenerate. The width of the above states is $0.3-0.4 \mathrm{eV}$ because they are highly localized with a small overlap of wave functions. Experimental evidence for the existence of the $e_{g}$ and $b_{1 g}$ orbitals and their spatial maps are reported in ref. (6). The former orbital is localized on the Pc ring and the latter orbital on the central part of molecule. Since these states can be found at $0.9-1.1 \mathrm{eV}$, so the intact molecule should appear in scanning tunneling microscopic (STM) images as a cloverleaf-shaped entity with well-defined lobes and core at a bias voltage of $1 \mathrm{~V}$ and higher.

Berkelaar et al. studied $\mathrm{CuPc}$ molecules adsorbed on $\mathrm{Au}$ induced $\mathrm{Ge}(001)$ nanowires using an STM (76). The $\mathrm{Au} / \mathrm{Ge}(001)$ surface contains well-ordered arrays of linear $\mathrm{Au}$-induced nanowires. The spacing between the adjacent nanowires is such, i.e., $1.6 \mathrm{~nm}$, that the $\mathrm{CuPc}$ molecules can nicely bridge the gap between two adjacent nanowires. Out of the six adsorption configuration identified in ref. (76), four of them consist of molecules bridging two adjacent nanowires. These four adsorption configuration were referred to as "molecular bridge" configurations. Here, we investigate electronic and dynamic properties of single $\mathrm{CuPc}$ molecule adsorbed in a molecular bridge configuration where two lobes of the molecule bind to one nanowire and the other two lobes to the adjacent nanowire. As the depth of the troughs between the nanowires is at least four atomic layers as reported in several papers $(59,67,68)$, it implies that $\mathrm{Cu}$ core of the $\mathrm{CuPc}$ molecules in the molecular bridge configuration is structurally fully decoupled from the underlying $\mathrm{Au} / \mathrm{Ge}(001)$ substrate. The electronic properties of the $\mathrm{CuPc}$ molecules are studied using scanning tunneling spectroscopy (STS) and dynamics using time-resolved current spectroscopy.

\subsection{Experimentation}

$\mathrm{Ge}(001)$ samples were cleaned by prolonged $500 \mathrm{eV} \mathrm{Ar}^{+}$ion sputtering at room temperature and flash annealing via resistive heating at $1100 \pm 25 \mathrm{~K}$. 
After several cleaning cycles, the $\mathrm{Ge}(001)$ samples were atomically clean and exhibited a well-ordered $(2 \times 1) / c(4 \times 2)$ domain pattern (77, 78). Subsequently, a monolayer of $\mathrm{Au}$ was deposited on the $\mathrm{Ge}(001)$ substrate at room temperature. After Au deposition the sample was annealed at $650 \pm 25 \mathrm{~K}$ for several minutes and inserted into the low-temperature (LT) STM for imaging. The $\mathrm{CuPc}$ molecules were deposited on the $\mathrm{Au} / \mathrm{Ge}(001)$ substrate using a Knudsen cell with a quartz crucible. The used $\mathrm{CuPc}$ was $>99 \%$ pure. The sample was directly facing the Knudsen cell's outlet at a distance of about 2 $\mathrm{cm}$. During the deposition the temperature of the Knudsen cell was kept at $573 \pm 5 \mathrm{~K}$, while the sample was held at room temperature. The deposited amount of $\mathrm{CuPc}$ varied from $1 \times 10^{12}$ to $5 \times 10^{12}$ molecules $/ \mathrm{cm}^{2}$. After deposition the sample was transferred from the preparation chamber to the STM chamber and cooled down to $77 \mathrm{~K}$. All results shown below were acquired at this temperature.

\subsection{Results and Discussion}

\subsubsection{Structural and Electronic Properties}

Figure 5.1 shows an overview of CuPc molecules adsorbed on $\mathrm{Au} / \mathrm{Ge}(001)$ surface. The molecules are randomly distributed on the surface at this coverage and do not assemble together into larger supramolecular structures. Among the several adsorption configurations observed in Fig. 5.1, the "molecular bridge" configuration is the most stable and abundant one (76). In this molecular bridge configuration, two lobes of a $\mathrm{CuPc}$ molecule (the structure is shown in the inset of Fig. 5.1 bind to one nanowire, while the other two lobes bind to the adjacent nanowire. All four lobes of the $\mathrm{CuPc}$ molecules appear bright in empty-state STM images. In filled state images, the lobes of the $\mathrm{CuPc}$ molecule show up as well, albeit with a somewhat lower resolution. The $\mathrm{CuPc}$ molecule appears a bit larger than $1.4 \mathrm{~nm} \times 1.4 \mathrm{~nm}$ because of the convolution with the STM tip.

More detailed information on the structural and electronic properties of $\mathrm{CuPc}$ in the molecular bridge position can be obtained from a high-resolution STM image and differential conductivity $\left(\frac{d I}{d V}\right)$ spectra, as shown in Figs. 5.2(a) and 5.2 (b), respectively. The STM image is recorded at a sample bias voltage of $1.5 \mathrm{~V}$. In this image, the core of the $\mathrm{CuPc}$ molecule, i.e., the $\mathrm{Cu}$ ion, appears as a "dark" spot, i.e., a depression. Since the trough between the nanowires 


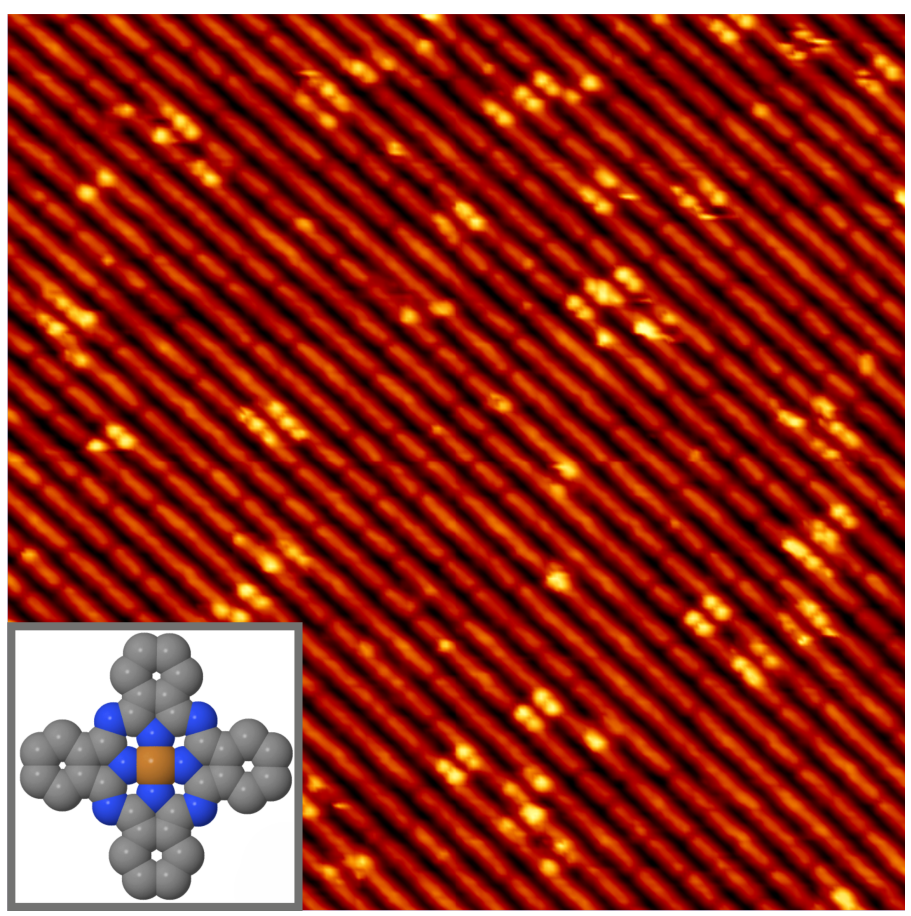

Figure 5.1: A large-scale STM image (42 $\mathrm{nm}$ x $42 \mathrm{~nm}$ ) of CuPc molecules adsorbed on $\mathrm{Au} / \mathrm{Ge}(001)$ surface recorded at $77 \mathrm{~K}$. The density of $\mathrm{CuPc}$ molecules is $2.8 \times 10^{12} \mathrm{~cm}^{-2}$. The Au-induced nanowires show up as bright lines running in the diagonal direction. The lobes of $\mathrm{CuPc}$ molecules appear as bright protrusions. Some molecules hop between different sites during imaging, leading to multi-lobed structures. The sample bias voltage is $1.5 \mathrm{~V}$ and the tunneling current is $0.2 \mathrm{nA}$. Inset: schematic model of a $\mathrm{CuPc}$ molecule. The orange, blue, and gray balls represent $\mathrm{Cu}, \mathrm{N}$, and $\mathrm{C}$ atoms, respectively. $\mathrm{H}$ atoms are not shown for the sake of simplicity.

has a depth of at least $6 \AA$, the core of the $\mathrm{CuPc}$ is at this position physically blocked from making direct contact to the underlying substrate (59). $\mathrm{CuPc}$ molecules in this configuration are very stable and do not exhibit any dynamics in contrast to the molecules where speckles are seen in the lobes as discussed later. The molecules with lobes containing speckles are susceptible to tunneling current induced changes such as diffusion and rotation (76). The STS spectra shown in Figs. 5.2 and 5.3 are recorded on $\mathrm{CuPc}$ molecules with stable lobes. It should be noted that these stable molecules are by no means a minority but make up to about $50 \%$ of all adsorbed $\mathrm{CuPc}$ molecules. The $\frac{d I}{d V}$ spectra reveal a clear difference in electronic properties of the core and lobes of 
the $\mathrm{CuPc}$ molecule in the molecular bridge configuration. The local density of states (LDOS) of the lobes exhibits a pronounced peak at $-1.62 \mathrm{~V}$ originating from the HOMO. This HOMO of the $\mathrm{CuPc}$ molecule has been studied earlier theoretically (111) as well as experimentally (113). The LDOS of the core of the $\mathrm{CuPc}$ molecule is significantly smaller and virtually featureless. The absence of the LUMO on the core of the $\mathrm{CuPc}$ molecule in Fig. 5.2(b) seems in disagreement with previous observations (109, 110). However, as will be discussed below this infers from specific properties of the $\mathrm{CuPc}$ molecule in the molecular bridge configuration.

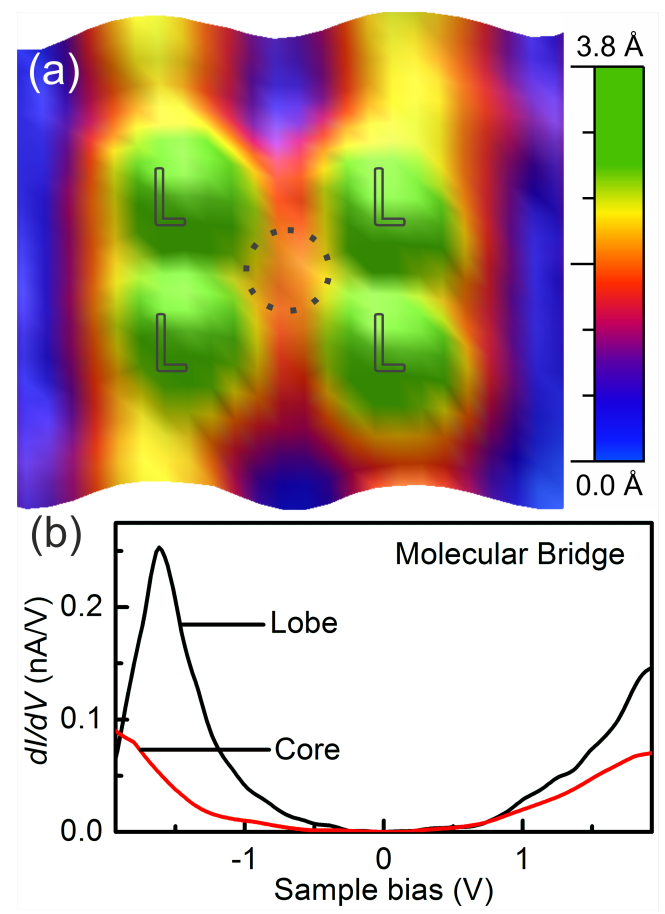

Figure 5.2: (a) 3D STM image $(3.4 \mathrm{~nm} \times 2.6 \mathrm{~nm})$ of a single $\mathrm{CuPc}$ molecule in the molecular bridge configuration. The sample bias voltage is $1.5 \mathrm{~V}$ and the setpoint is $0.2 \mathrm{nA}$. Four lobes of the molecule (labeled L) are bound to two Au-induced nanowires. The central part of the molecule, i.e. the $\mathrm{Cu}$ core (outlined by a dotted circle), appears as a "dark" spot. (b) Differential conductivity $\left(\frac{d I}{d V}\right)$ spectra recorded on the core and the lobes, respectively show non-metallic character at Fermi energy. The spectra are measured with a lock-in amplifier. The frequency is $3.98 \mathrm{kHz}$ and the modulation voltage is $10 \mathrm{mV}$. The setpoints is $0.4 \mathrm{nA}$ while the sample bias is $2.0 \mathrm{~V}$.

The structural and electronic properties of the $\mathrm{Au} / \mathrm{Ge}(001)$ system and $\frac{d I}{d V}$ 
spectra of the nanowires and the troughs are already published and discussed in detail in Refs. (59, 63 73, 114). A brief description of Au induced nanowires has been presented in chapter 3 . I-V spectra recorded on-top and in between the $\mathrm{Au}$ induced nanowires reveal that the troughs are slightly more metallic than the Au induced nanowires (65, 66, 114).

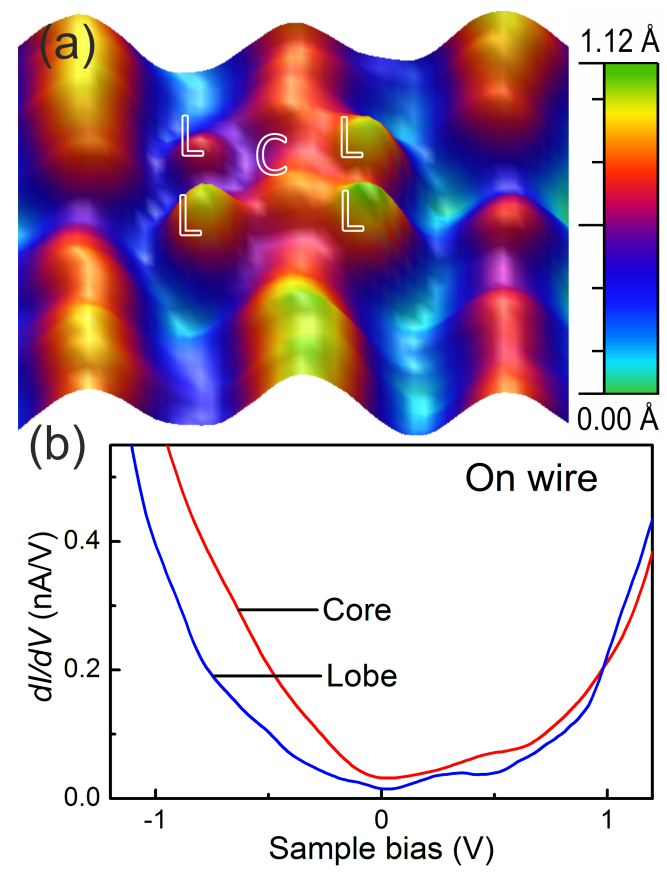

Figure 5.3: (a) $3 \mathrm{D}$ STM image $(4.1 \mathrm{~nm} \times 3.1 \mathrm{~nm})$ of a single $\mathrm{CuPc}$ molecule residing on-top of a nanowire ("on-wire" configuration). The molecule's core (labeled $\mathrm{C}$ ) binds to the nanowire, whereas the lobes (labeled L) are hanging in the adjacent troughs. Both core and lobes appear as well-resolved protrusions. The sample bias voltage is $1.5 \mathrm{~V}$ and the tunneling current is $0.3 \mathrm{nA}$. (b) $\frac{d I}{d V}$ spectra of the core and lobes of the $\mathrm{CuPc}$ molecule adsorbed in the on-wire configuration. The setpoints is $0.3 \mathrm{nA}$ while the sample bias is $1.5 \mathrm{~V}$.

The lack of any LDOS at the Fermi level $(0 \mathrm{~V})$ for both the spectra in Fig. 5.2 (b) demonstrates the nonmetallic character of the $\mathrm{CuPc}$ at the molecular bridge site. Obviously, the most striking observation is that the core of the $\mathrm{CuPc}$ molecule, i.e., the $\mathrm{Cu}$ atom, is fully decoupled from the substrate. To verify this unique property of $\mathrm{CuPc}$ in the bridge position, we studied the properties of a $\mathrm{CuPc}$ molecule residing at a different adsorption site.

Figure 5.3(a) shows an STM image of a CuPc molecule adsorbed on-top of 
a nanowire. In this adsorption configuration, which was not reported earlier (76), the core is located on-top of the nanowire and the lobes are lying in the adjacent troughs. This configuration is referred to as "on-wire" adsorption configuration. In the STM image, the lobes appear slightly brighter than the core of the molecule in the form of four separate well-resolved protrusions. In contrast to the $\frac{d I}{d V}$ spectra of the $\mathrm{CuPc}$ molecule in the molecular bridge configuration, the $\frac{d I}{d V}$ curves depicted in Fig. 5.3.(b) for the on-wire adsorption site reveal the metallic nature of the core as well as the lobes, although the core is slightly more metallic than the lobes.

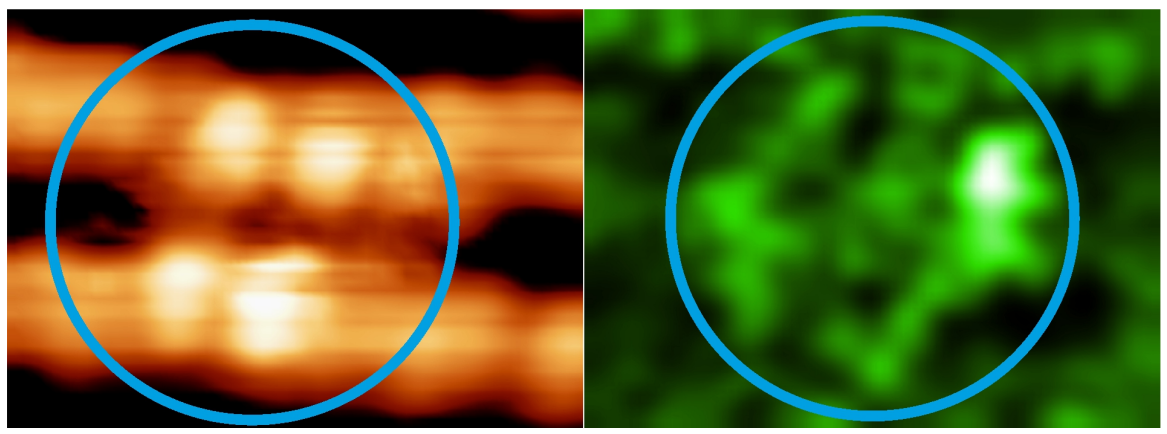

Figure 5.4: Topography (left) and $\frac{d I}{d V}$ map (right) recorded simultaneously at $2.0 \mathrm{~V}$ (set point current $=0.2 \mathrm{nA}$ ) using a lock-in amplifier. The highest LDOS is located at the periphery of the Pc macrocycle.

By comparing the data presented in Figs. 5.2 and 5.3 , one can clearly rationalize the difference between the $\mathrm{CuPc}$ molecules adsorbed at the molecular bridge and on-wire configurations. In the latter configuration, the $\mathrm{Cu}$ core can directly interact with atoms of the nanowire. According to the structural model given van Houselt et al. (59), the ridge consists of mixed dimers of $\mathrm{Au}$ and Ge atoms. Due to a difference in electronegativity of $\mathrm{Cu}(1.90)$ and $\mathrm{Ge}$ and $\mathrm{Au}$ (2.01 and 2.54, respectively), charge transfer can in principle occur from the $d$ orbital of the $\mathrm{Cu}$ core to the nanowire, e.g., to the dangling bonds of the Ge or Au atoms (59). This makes the molecular core coupled to the underlying nanowire. Moreover, this interaction will affect the tails of the LUMO and HOMO states. The aforementioned scenarios will result in a well-defined protrusion in the center of the molecule, as shown in Fig. 5.3. (a). In addition, it should be noted that filling of the $d$ orbital of the MPc molecules can drastically affect the character of this orbital (115). We therefore anticipate that due to coupling of the $\mathrm{CuPc}$ molecule to the nanowire, the $d$ orbital of the 
molecule will have a more pronounced $z$-character as compared to the isolated $\mathrm{CuPc}$ molecule which exhibits a $d$ orbital with a strong $d_{x^{2}-y^{2}}$ character.

In contrast, the $\mathrm{CuPc}$ in the molecular bridge position has, as described above, a lower LDOS at the core as compared to the lobes suggesting that the core is also electronically decoupled from the underlying metallic trough (69). Figure 5.4 shows an STM image of CuPc in the molecular bridge configuration as well as spatial map of the differential conductivity at a sample bias of 2.0 $\mathrm{V}$. The highest LDOS is measured at the lobes of the $\mathrm{CuPc}$ molecule.

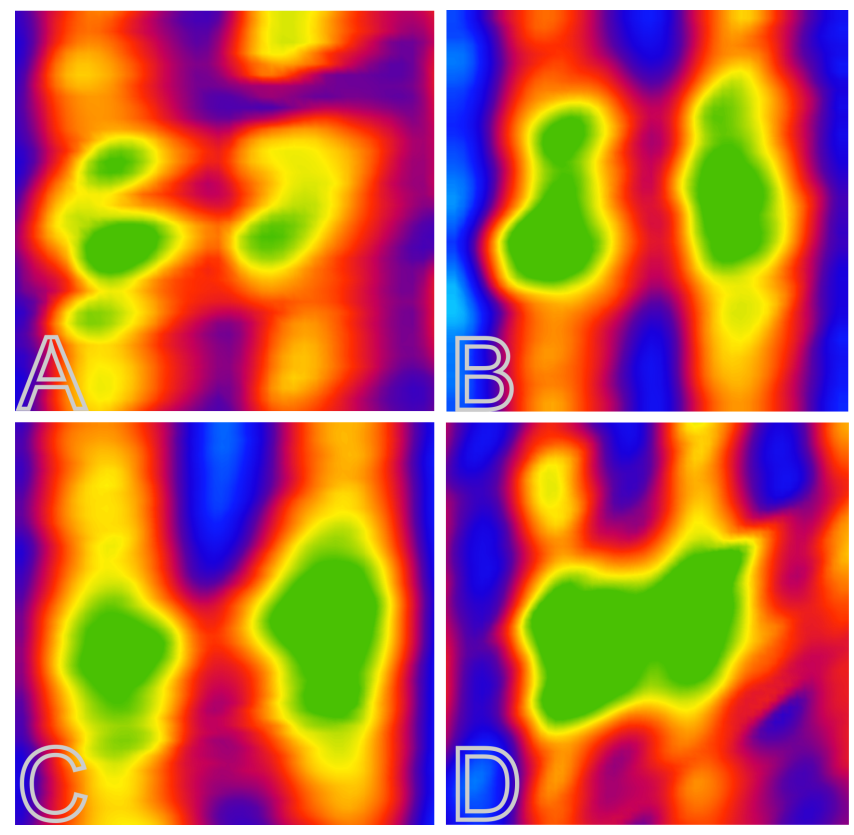

Figure 5.5: (A) $\mathrm{CuPc}$ molecules in different configurations are imaged at bias voltages as low as $0.5 \mathrm{~V}$. A series of empty-state STM images of the CuPc molecule adsorbed at molecular bridge site demonstrate charging of the molecules at sample bias $\mathrm{V}_{S}>3.5 \mathrm{~V}$. (B) CuPc molecule at sample bias $\mathrm{V}_{S}=1.5 \mathrm{~V}$ and $(\mathrm{C})$ at $3.5 \mathrm{~V}$, where the core appears as a depression. (d) However, at $\mathrm{V}_{S}=5.0 \mathrm{~V}$, the core of the molecule is as bright as its lobes indicating that the core of the molecule is charged at this bias voltage.

In Fig. 5.5, a series of STM images of the CuPc molecule in the molecular bridge configuration at various bias voltages in range of $0.5 \mathrm{~V}$ to $5 \mathrm{~V}$ is shown. In the STM images recorded at $0.5 \mathrm{~V}, 1.5 \mathrm{~V}$, and $3.5 \mathrm{~V}$ as shown in Figs. 5.5(A), (B) and (C), respectively, the core appears as a "dark" spot in the center of the molecule, whereas the lobes of the molecule are well-resolved, but 
at $5 \mathrm{~V}$ the core of the molecule pops up in the STM image as shown in Fig. 5.5(D). Therefore, we conclude that charging of a single $\mathrm{Cu}$ ion (i.e., decoupled $\mathrm{Cu}$ core) occurs somewhere in between $3.5 \mathrm{~V}$ and $5.0 \mathrm{~V}$. This threshold value is more than $3 \mathrm{~V}$ higher than the LUMO of the isolated $\mathrm{CuPc}$ molecule, which is located 0.6-0.9 eV above the Fermi level (109, 110). This high charging energy $\left(\frac{e^{2}}{2 C}\right)$ makes sense since the capacitance $(C)$ of the core of $\mathrm{CuPc}$ is very small (116). It is important to note that at these large sample biases the adsorption geometry of the $\mathrm{CuPc}$ molecule does not change, as shown in Fig. 5.5. However, the structure of a molecule can in principle be changed in its charged state, for example, the Zn(II) Etioporphyrin I molecule adsorbed on NiAl changes its chirality upon charging (117). Such structural change is ruled out in our case as we do not observe any detectable change in the structure of the molecule except for the fact that the core of $\mathrm{CuPc}$ becomes more bright. We can also rule out the possibility of dehydrogenation of the lobes of $\mathrm{CuPc}$ molecules as observed by Zhao et al. (118). They point out that the dehydrogenation of lobes takes place at about 3.2-3.6 V accompanied by a drop in the current by a factor of 2 and consequently the lobes become dim. However, as shown in Fig. 5.5, the appearance of the lobes of the $\mathrm{CuPc}$ molecule does not change when changing the bias from $1.5 \mathrm{~V}$ to $5 \mathrm{~V}$. So, we can safely conclude that dehydrogenation does not take place in our case.

\subsubsection{Dynamics of CuPc Molecules}

Single molecules adsorbed on surfaces can exhibit dynamics where the molecular motion can be driven thermally (119) or by electrons injected by an STM tip (120, 121). In chapter 6 , we will describe dynamics of $\beta$-cyclodextrin $(\beta-C D)$ molecules in self-assembled monolayer on $\mathrm{Au}(111)$ surface. Such dynamics is related to the conformational changes of the $\beta$-CD molecules induced by electrons which tunnel inelastically. $\mathrm{CuPc}$ molecules on the $\mathrm{Au} / \mathrm{Ge}(001)$ surface also exhibit dynamics as shown by Berkelaar et al. (76) using STM and time-resolved spectroscopy. They demonstrate an interesting rotational dynamics of $\mathrm{CuPc}$ molecules adsorbed on the $\mathrm{Au} / \mathrm{Ge}(001)$ nanowires where the molecule changes back and forth between two adsorption configurations.

Here, we observe that the $\mathrm{CuPc}$ molecules show a lateral diffusion on the $\mathrm{Au} / \mathrm{Ge}(001)$ surface by hopping from one position to another. The molecule diffuses away from the encircled area in subsequent scanning as shown in the Fig. 5.6. Such lateral diffusion is induced by electrons that tunnel inelastically. 

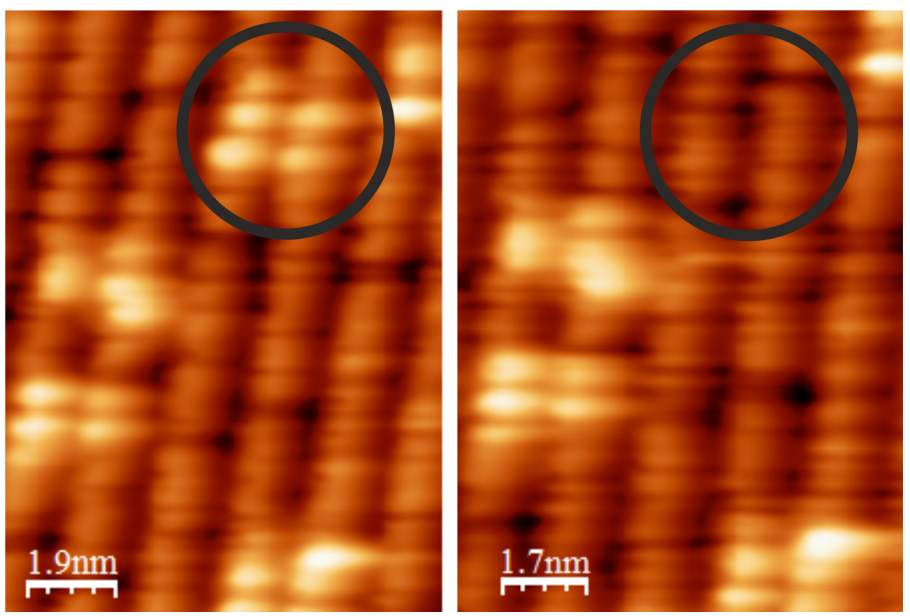

Figure 5.6: Two subsequent STM images show lateral diffusion of CuPc molecule on the surface. These dynamic events are observed more frequently when scanning areas are smaller for same scanning rate and tunneling parameters. This indicates that this dynamics is induced by tunneling electrons. The images were recorded at setpoint current of $0.2 \mathrm{nA}$ and bias voltage of $1.5 \mathrm{~V}$.

An indication of electron induced dynamics can be obtained by witnessing an enhanced number of such dynamic events as the scan size is reduced. For a given setpoint current and scanning rate, scanning a smaller area results in a larger charge transfer per unit area through the molecules which will give rise to a larger number of dynamic events.

Furthermore, we also observe the presence of molecules with lobes having speckles as shown in the encircled area in the top panel of Fig. 5.7. The speckles are attributed to changes in the tunneling current due to lateral dynamics of the lobes (26) of the molecule. The CuPc molecule shown in Fig. 5.7 has only three lobes in contrast to the well-defined four lobe stable $\mathrm{CuPc}$ molecule (as can be seen in the left part of the top panel). The four lobe stable $\mathrm{CuPc}$ molecules does not show speckles and consequently the current-time (I-t) traces recorded at the lobes do not exhibit any jumps (not shown). This is also true for $\mathrm{CuPc}$ molecule on-top configuration where no speckles have been observed. The I-t spectroscopy shown in Fig. 5.7 (bottom panel) is one of the typical spectra recorded on the lobes with speckles. It is evident that from the two current levels that the lobe toggles back and forth between a high current level and a low current level. An analysis of few I-t spectra reveal that the average residence time of low current state lies in the range of 3 to 9 


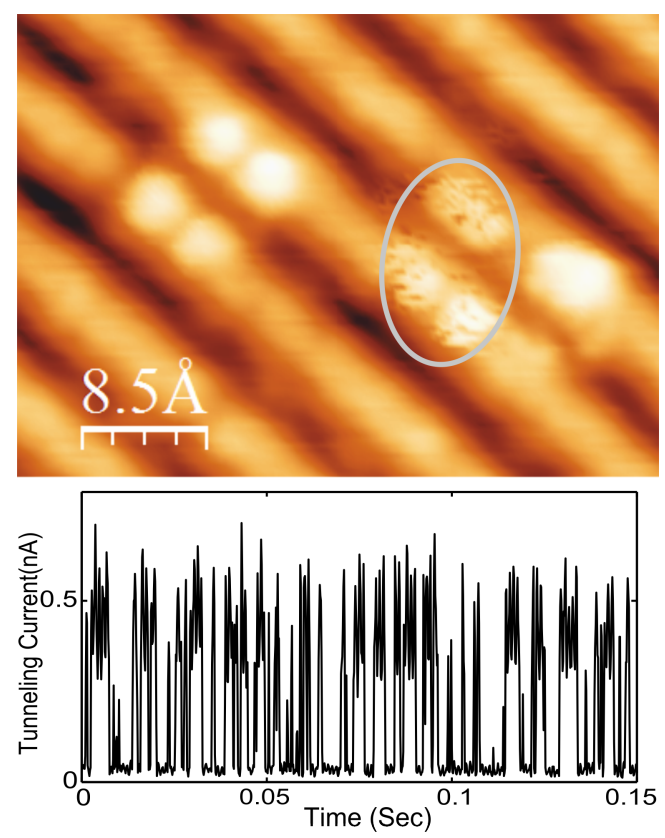

Figure 5.7: The lobes of the $\mathrm{CuPc}$ molecules show up as bright protrusions, however, some molecules have lobes with speckles as in the encircled area shown in the top panel. I-t traces recorded on these lobes with speckles clearly exhibit a strong dynamic behaviour as shown in the bottom panel. $\mathrm{CuPc}$ molecules with well-defined lobes (for instance, the $\mathrm{CuPc}$ molecule in the left part of the top panel) are static and do not exhibit any dynamics.

ms and the residence times follow Poisson distribution. Poisson distribution means that the toggling process is fully stochastic.

The speckles observed in the lobes are very similar to that observed for two opposite lobes of $\mathrm{CuPc}$ molecules deposited on the $\mathrm{Cu}(111)$ surface (26). In these experiments, the dynamics is induced by the electrons that tunnel inelastically. Schaffert et al (26) attributed this dynamics to the transfer of angular momenta of the tunneling electrons to the resonant molecular state. The lateral dynamics of the lobes of the $\mathrm{CuPc}$ molecule on the $\mathrm{Au} / \mathrm{Ge}(001)$ surfaces is inferred from the fact that the lobes with speckles appear considerably larger than the lobes of stable $\mathrm{CuPc}$ molecule. Finally, it is worth mentioning that the elongation of lobes with speckles systematically occurs along the direction of the nanowires. This is consistent with our interpretation that the observed dynamics is due to the fact that the lobes jump back and forth between two adjacent adsorption sites on the nanowires. 


\subsection{Conclusion}

In this chapter, we have demonstrated that we are able to structurally and electronically decouple the core of a $\mathrm{CuPc}$ molecule, i.e., the double ionized $\mathrm{Cu}$ ion, from the underlying substrate. This was achieved by trapping the molecule in a molecular bridge position between two adjacent Au-induced nanowires on $\mathrm{Ge}(001)$. The core of the $\mathrm{CuPc}$ molecule is invisible in emptystates STM images up to a sample bias of $3.5 \mathrm{~V}$. Charging of the core occurs at sample biases larger than $3.5 \mathrm{~V}$, as has been directly visualized in normal STM images. More generally, MPc molecules on the $\mathrm{Au} / \mathrm{Ge}(001)$ surface can be considered as a model system in which the core of the molecule with the desired functionalization (e.g., with a magnetic moment) can remain intact, while its ligands are contacted to the macroscopic outside world. The amount of control over the $\mathrm{CuPc} / \mathrm{Au} / \mathrm{Ge}(001)$ system, we have demonstrated here, opens a novel avenue for better understanding of essential ingredients of molecular electronics, which may enhance its realization. 
5. Electronic Properties of a CuPc Molecule in a "Molecular Bridge" Configuration 


\section{Chapter 6}

\section{Electron Induced Dynamics of $\beta$-Cyclodextrin Molecules}

In this chapter, we study variable temperature scanning tunneling microscopy (STM) and spectroscopy (STS) measurements on hepthathioether $\beta$-cyclodextrin $(\beta-C D)$ self-assembled monolayers (SAM) on Au(111) surfaces. The $\beta-C D$ molecules exhibit very rich dynamical behavior, which is not observed in ensemble averaged studies using macroscopic junctions such as eutectic GaIn technique. The dynamics is reflected in the tunneling current-time traces, which were recorded with the STM feedback loop disabled. The dynamics is temperature independent, but increases with increasing tunneling current and sample bias, indicating that the conformational changes of the $\beta$-CD molecules are induced by electrons that tunnel inelastically. Even for sample biases as low as $10 \mathrm{mV}$ we observe well-defined levels in the tunneling current-time traces at temperature of $77 \mathrm{~K}$. We attribute these jumps to the excitations of the molecular vibration of the macrocyclic $\beta-C D$ molecule. Current-voltage measurements reveal oscillations in the differential conductance traces which correspond to field emission electronic resonance states. These oscillations have also been not reported in case of eutectic GaIn junctions. Therefore, a great care must be taken in the studies of macroscopic junction devices formed on self-assembled monolayer. 


\subsection{Introduction}

To meet the great expectations in the field of molecular electronics (15, 99, 122, 123), we must possess a sound understanding of charge transport through the individual components in the molecular system. Even though a large variety of approaches have been used to create molecular junctions (5, 123), there are currently only a few examples of unambiguous charge transport studies in literature (124-127). A majority of the large area devices created and investigated are based on self-assembled monolayers (SAMs) immobilised between two macroscopic electrodes (15, 127-130). However, due to the macroscopic nature of the metal-monolayer contacts, the current-voltage (I-V) data obtained always presents an average of the charge transport events that occur across the array of contacted molecules. Therefore, a detailed understanding of charge transport across single molecules cannot be achieved when investigating the phenomena that occur in molecular assemblies (10, 21, 25, 83, 131). There have also been limited efforts to interpret the stochastic events (22, 132), but a clear understanding of transport through individual entities is still difficult to infer from such large ensembles.

Recently, the I-V measurements of supramolecular tunneling junctions were reported where dendrimers of various terminal functionality, multivalently absorbed on a supramolecular platform of hepthathioether $\beta$-cyclodextrin $(\beta-C D)$ were immobilized on template stripped Au substrate. Here, the top contact was applied via the $\left(\mathrm{Ga}_{2} \mathrm{O}_{3}\right)$ EGaIn technique (17). EGaIn is an eutectic mixture of $\mathrm{Ga}$ and $\mathrm{In}(75 \%$, and $25 \%$ by weight, respectively) and has a melting point of $15.5{ }^{\circ} \mathrm{C}$, just below the room temperature (15). As the EGaIn forms a conformal, low-resistance, non-damaging, non-toxic, micrometer sized contact to SAMs surfaces, it was used to study the statistical average of transport properties of the $\beta$-CD SAM (17). The schematic of the EGaIn setup is shown in Fig. 1.1(C) in chapter 1.

$\beta-C D$ superamolecular systems are interesting as they can act as host molecules for a variety of organic/inorganic molecules for sensing applications and molecular electronics applications (17, 133). These supramolecular systems have been previously characterised with atomic force microscopy (AFM) (133-135), electrochemistry, cyclic voltammetry (CV) (133, 136-138), electrochemical impedance spectroscopy (EIS) (133, 138), differential pulse voltammetry (DPV) (137-139), scanning electrochemical microscopy (SEM) 
(140), surface plasmon resonance (SPR) (134, 137, 138), and SPR and CV combined (137, 138). Electronic transport properties of $\beta$-CD monolayer was studied using large area EGaIn junctions. However, the true mechanism of charge transport remains unclear. As in many of the other tunneling junctions investigated our metal-monolayer contacts are of the macroscopic level, and therefore in an attempt to further understand the mechanism of charge transport through our assemblies we will investigate these phenomena at the level of single molecules, i.e with a scanning tunneling microscope (STM).

STM is commonly used to study the current-voltage characteristics of molecular self-assemblies. However, due to the limited temporal resolution of STM (a few seconds), dynamic events are often averaged out. The temporal resolution of a standard STM can be improved by 6-7 orders of magnitude by recording tunnel current-time traces (time-resolved scanning tunneling spectroscopy (STS) measurements) at a pre-selected position, while the feedback loop is switched off. Here, the bandwidth of the current-voltage converter $(\sim 100 \mathrm{kHz})$ limits the temporal resolution to $10-20 \mu \mathrm{s}$. Using the time resolved technique, conductance switching and motion of single oligo (phenylene ethynylene) molecules in the SAM matrix of dodecanethiolate have been studied in real time (141). Other examples of time-resolved STM measurements include molecular rotors of butyl sulphide (142) (t-Bu) $)_{4} \mathrm{ZnPc}$ (143), flipping dimers (37, 144-146), and flickering of single diarylethene molecules (147). All these studies target a single molecule on a surface where the dynamical and conformational events have been studied (43, 97). However, there have not been any time-resolved STM studies on molecular self-assemblies yet.

The dynamical events are reflected in the I-V measurements recorded on $\beta$-CD SAM showing characteristic jumps in the traces. In this chapter we use time-resolved STM measurements to investigate the molecular dynamics and address the electron coupling and vibronic modes of the $\beta$-CD molecules immobilized on $\mathrm{Au}$ surfaces. The I-V spectra also show a clear oscillations which result in peaks in the differential conductance curves. These oscillations indicate Fowler-Nordheim tunneling at relatively lower sample bias giving rise to Gundlach oscillations. The STM and STS measurements done on $\beta$-CD SAM brings a greater understanding about the charge transport within this molecular system. 


\subsection{Experimentation}

\subsubsection{Sample Preparation}

The synthesis of the heptathioether $\beta$-cyclodextrin $(\beta-C D)$ molecules was carried out using a process which is described in references (10, 148). All glassware used in the preparation of the $\beta$-CD monolayer was immersed in a piranha solution $\left(\mathrm{H}_{2} \mathrm{SO}_{4}\right.$ and $33 \% \mathrm{H}_{2} \mathrm{O}_{2}$ in a $3: 1$ ratio) for a minimum of 20 minutes. The glassware was then rinsed with large amounts of Milli-Q water $(18.2 \mathrm{M} \Omega-\mathrm{cm})$, and dried in an oven at $400 \mathrm{~K}$. Glass supported Au substrates $(\mathrm{Au}(250 \mathrm{~nm}) / \mathrm{Cr}(2.5 \mathrm{~nm}) /$ Glass $)$ were purchased from arrandee ${ }^{\mathrm{TM}}$, Werther, Germany. The Au substrates were rinsed with dichloromethane, dried and subsequently flame annealed in a hydrogen flame to obtain grains with large (111) oriented Au terraces. To prepare the self-assembled monolayer (SAM) of $\beta-C D$, the $\mathrm{Au}$ substrates were immersed into a $0.1-1 \mathrm{mM}$ solution of $\beta$-CD dissolved in a chloroform-ethanol (1:2) solution for 16 hours at $350 \mathrm{~K}$. The substrates were removed and rinsed thoroughly with three cycles of dichloromethane, ethanol and Milli-Q water and then dried in a stream of nitrogen.

\subsubsection{Time-resolved Scanning Tunneling Spectroscopy}

The STM and time-resolved measurements on the $\beta$-CD SAMs were carried out with the RHK UHV700 and the Omicron Low Temperature STM (LT-STM) system under ultra-high vacuum (UHV) conditions. The RHK UHV700 system was used for room temperature measurements, while we used the Omicron LT-STM for the measurements at $77 \mathrm{~K}$. For time-resolved measurements (I-t), the tunneling current was recorded as a function of time, while the feedback loop was switched off. Sampling frequencies in the range from 1-10 KHz were used. We used electrochemically etched W-tips for all the measurements. A total of more than $900 \mathrm{I}-\mathrm{t}$ traces were recorded at room temperature and at 77 K. Each I-t trace was acquired for 5-30 seconds. About $75 \%$ of the I-t traces exhibited switching events between two or more current levels. Only a few percent of the I-t traces were useless due to the thermal drift of the tip with respect to the surface. These I-t traces are not included in our analysis. 


\subsection{Results and Discussion}

In this section we present and discuss STM imaging of the bare flame annealed $\mathrm{Au}(111)$ surface, the $\beta$-CD SAM on the $\mathrm{Au}(111)$ surface, and the I-V measurements performed on the $\beta$-CD SAM. This will be followed by the time resolved STM measurements performed on the $\beta$-CD SAM which display molecular dynamics occurring at both room temperature and $77 \mathrm{~K}$. The amount of these dynamic events increases with increasing tunneling current. We discuss our hypotheses for the observed dynamics of the $\beta$-CD molecules. Current-voltage (I-V) measurements also show oscillations which result in well-positioned peaks in differential conductance. We find out that these peaks correspond to Gundlach oscillations as the transport falls in the regime of Fowler-Nordheim tunneling.

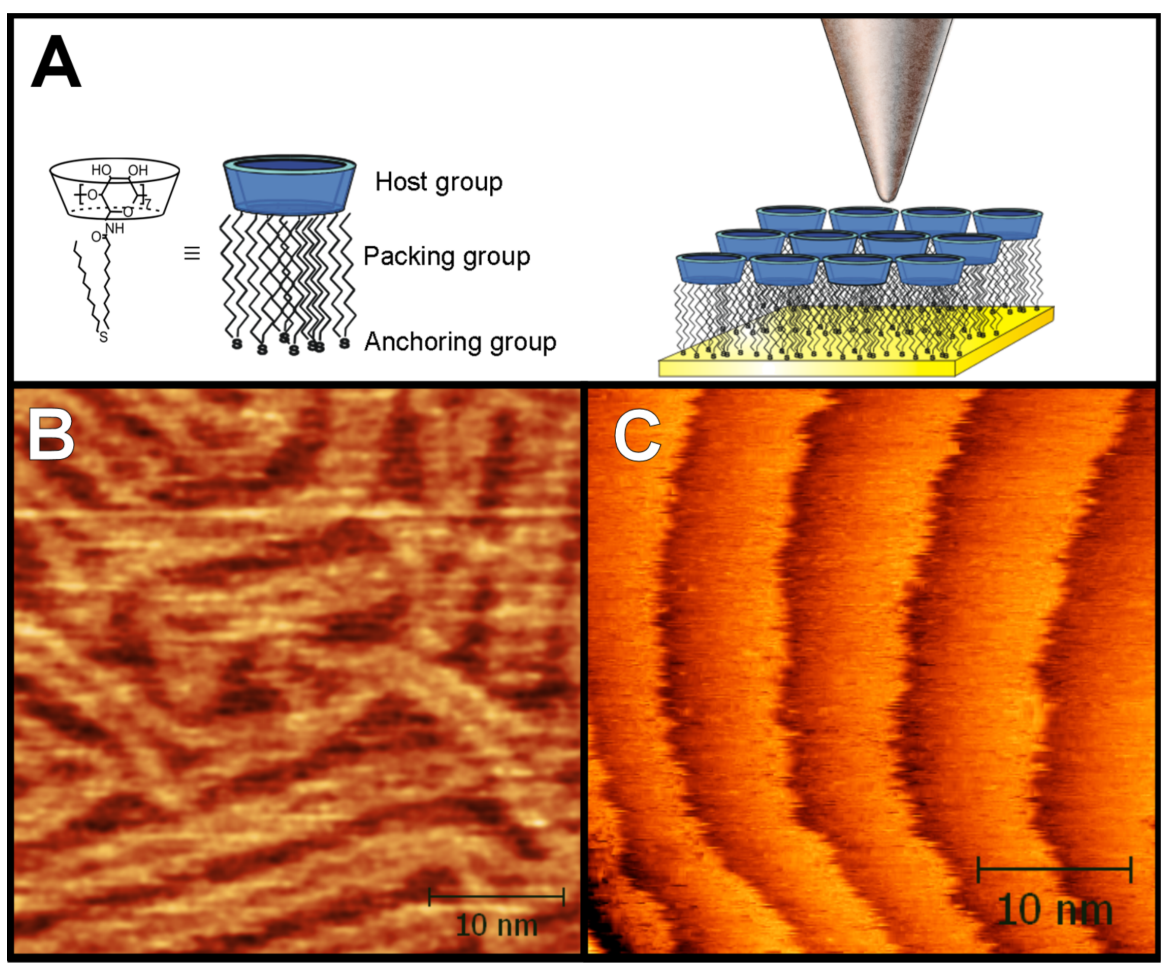

Figure 6.1: (A) Chemical structure of the $\beta$-CD molecule and a cartoon depicting the arrangement of $\beta$-CD molecules on an Au surface including an STM tip (see refs. (133) and (148)). (B) STM image of a bare $\mathrm{Au}(111)$ surface after flame annealing. (C) STM image of a $\beta$-CD self-assembled monolayer on $\mathrm{Au}(111)$ surface. 


\subsection{1 $\beta$-Cyclodextrin Self-Assembled Monolayer}

Figure 6.1(A) shows the molecular structure of a $\beta$-CD molecule (133, 148) and a cartoon depicting the arrangement of $\beta$-CD molecules in a SAM on a $\mathrm{Au}(111)$ surface with an STM tip positioned at the top. To ensure that the SAMs were formed on well annealed $\mathrm{Au}(111)$ surfaces, the Au substrates were introduced into the UHV system and imaged. Figure 6.1(B) is an STM image of the bare Au substrate. The pattern on the terraces is due to the presence of a herringbone reconstruction of the $\mathrm{Au}(111)$ surface, thus indicating very well annealed Au surfaces. The ordering of the herringbone reconstruction is slightly more irregular than that of an UHV cleaned $\mathrm{Au}(111)$, but the presence of this on a flame annealed Au surface is still exceptional.

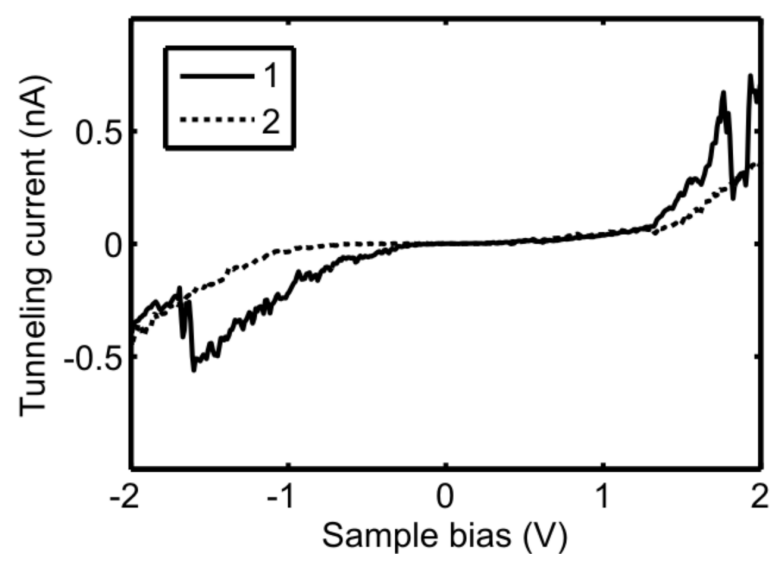

Figure 6.2: I-V curves of the molecular tunnel junction recorded at room temperature. The setpoint is $2 \mathrm{nA}$ and the sample bias is $-3 \mathrm{~V}$. I-V trace 2 does not exhibit any conductance change, whereas I-V trace 1 jumps back and forth between a low current value and a high current value. The low current value overlaps with $\mathrm{I}-\mathrm{V}$ trace 2.

Figure 6.1(C) shows an STM image of the $\beta$-CD SAM recorded at room temperature under UHV conditions. The measured step height of $0.25 \mathrm{~nm}$ corresponds to the height of a single layer step of $\mathrm{Au}(111)$. We did not achieve molecular resolution on the terraces. This was not caused by a thermally induced motion of the $\beta$-CD molecules, as imaging at $77 \mathrm{~K}$ also did not produce molecular resolution. This inability to obtain molecular resolution can be due to intermolecular transport parallel to the substrate. Even though molecular resolution is not obtained the $\mathrm{I}-\mathrm{V}$ measurements recorded on the $\beta-\mathrm{CD}$ 
SAM are substantially different from the I-V traces recorded on the bare $\mathrm{Au}$ substrate. Therefore, we conclude that the $\beta$-CD SAM is indeed present. I$\mathrm{V}$ traces recorded at the same location on the $\beta$-CD SAM are shown in the Figure 6.2. Both I-V traces show an exponential behavior with different exponent decays. At higher bias values, I-V trace 1 jumps to lower current values, which overlaps with I-V trace 2 . After the jump to a lower current value, it again jumps back to the original value at a positive bias. That means that the molecular assembly shows a conductance change during the acquisition of the I-V traces. This may be related to the molecular dynamics occurring in the SAM. In order to study the nature of such dynamics we performed time-resolved STM measurements as discussed in the next section.

\subsubsection{Dynamics of $\beta$-Cyclodextrin Molecules}
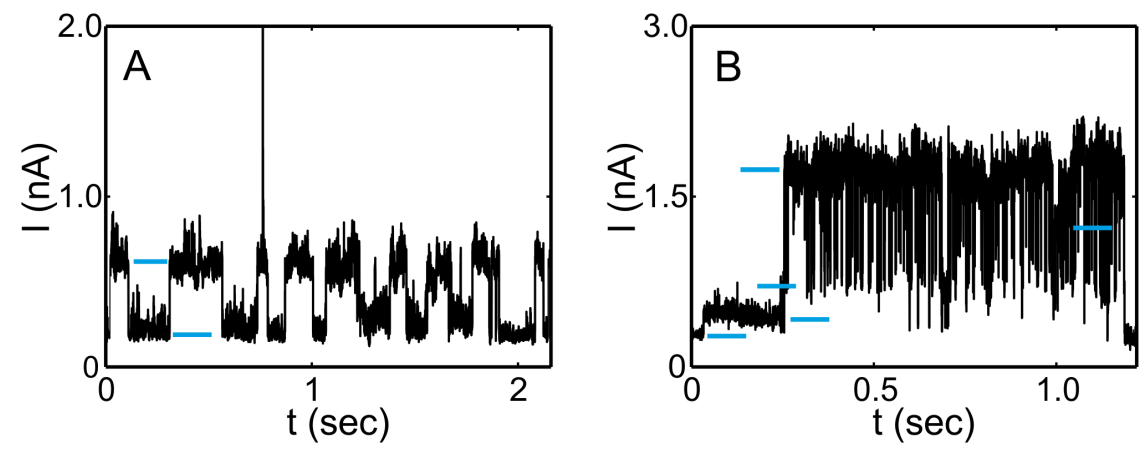

Figure 6.3: (A)-(B) Current-time (I-t) traces performed on a $\beta$-CD self-assembled monolayer at $300 \mathrm{~K}$. The sample bias was $-1.25 \mathrm{~V}$. The blue marks indicate various current levels.

Figure 6.3 shows two time-resolved STM measurements recorded at room temperature, only at areas where flat terraces are present. In Figure 6.3(A) the $\beta$-CD molecule switches back and forth between a low current level of $\approx 0.3 \mathrm{nA}$ and a high current level of $\approx 0.6 \mathrm{nA}$. We believe that these two levels correspond to two different conformational configurations of the molecule. There are several possible causes for such dynamics observed in the timeresolved traces. For example, the X-ray Photoelectron Spectroscopy (XPS) studies (148) carried out on $\beta$-CD SAMs showed that on average only 3.2 out of the 7 sulfur (S) moieties (anchoring groups) are bound to Au at any one time. Therefore, during the period of time that the STM measurement is 
performed, the number of $\mathrm{S}$ moieties of the $\beta$-CD molecule bound to the $\mathrm{Au}$ surface could change, for example from $3 \mathrm{~S}$ - $\mathrm{Au}$ interactions to $4 \mathrm{~S}-\mathrm{Au}$ interactions, due to the labile bonding between the sulfur and the Au (149). Also the sulfur moieties can have different adsorption geometries on the Au surface which is directly related to the conductance through the anchoring groups of the molecules. Therefore, the attachment and de-attachment of the anchoring groups with different adsorption geometries will result in many levels.
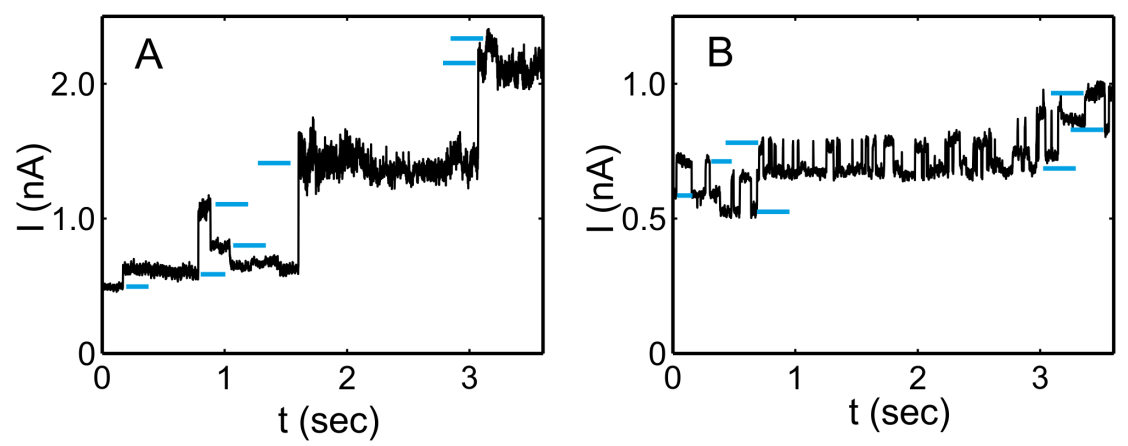

Figure 6.4: (A)-(B) Current-time (I-t) traces performed on a $\beta$-CD self-assembled monolayer at $77 \mathrm{~K}$ show at least seven different levels. The sample biases were $-0.25 \mathrm{~V}(\mathrm{~A})$ and $-1.25 \mathrm{~V}(\mathrm{~B})$, respectively. The blue marks indicate various current levels.

Intermolecular interactions could also be a reason for the observed switching. As mentioned above these interactions seem to be important as they may be the reason we do not achieve molecular resolution on the surface. The intermolecular interactions can be due to the van der Waals force, or polarization effects which can also lead to the conformational changes in the molecules. One example of multi-level conductance switching is shown in Figure 6.3.(B). The residence times in the two high current levels are much shorter than the residence times of the low current levels. An analysis of the distribution of residence times of the two high current levels reveals that the switching process between the two levels is fully stochastic, i.e. there is no memory effect. The average residence times at these two levels are $\sim 3.6 \mathrm{~ms}$ and $\sim 0.57 \mathrm{~ms}$, respectively. This difference is most likely due to an energetic asymmetry between the two higher conductance states, as will be discussed below and in Figure 6.6.

Various I-V curves recorded on the $\beta$-CD SAM at temperature $77 \mathrm{~K}$ also 
show similar jumps as shown in Figure 6.2. Therefore, we also expect the $\beta$-CD molecules to exhibit dynamic events in the I-t spectroscopy. Figure 6.4 shows two I-t traces recorded at $77 \mathrm{~K}$. The observed dynamics are similar to what is seen at room temperature and therefore the switching behavior of the $\beta-\mathrm{CD}$ is not thermally induced. In Figure 6.4(A), an I-t trace is shown that exhibits at least seven different current levels. The residence times in all levels are larger than $\approx 100 \mathrm{~ms}$. Figure 6.4(B) shows another I-t trace where seven current levels are observed. The residence times are however much shorter as compared to the trace in Figure 6.4(A). Although Figures 6.4(A) and (B) both exhibit at least seven different levels (small grey lines), the relative ratios of the different current levels and average residence times reveal that we are dealing with different conformational configurations of the $\beta$-CD molecule. However, we cannot be sure about the exact number of molecules involved in the tunneling process, due to the possibility of lateral transport, so it is also possible that the slow and fast switching processes are related to different molecules.

The recorded I-t traces reveal that the switching process is often reversible. The involved intermediate states might however, vary from trace to trace. The reversibility of the process indicates that the molecule switches between different but rather stable conformational configurations. A similar behavior has been observed for azobenzene (150), which switches between two isomers.

Since the observed dynamics is not thermally induced we have varied the tunneling current and sample bias in order to confirm if the dynamics are induced by electrons that tunnel inelastically. We found that the dynamics of the $\beta$-CD molecules become increasingly richer, i.e. more levels, faster, and shorter residence times, upon increasing tunneling current. The number of electrons that are required in order to induce a transition can in principle be determined by plotting the logarithm of the switching rate versus the logarithm of the current $\left(\right.$ rate $\left.\approx \mathrm{I}^{N}\right)$. The slope gives the order, $N$, i.e. the number of electrons that is required in order to induce a transition. Since the $\beta-C D$ molecules exhibit so many different levels, which we are unable to relate to specific conformational configurations of the molecule, we cannot determine the exact order of the transition.

In Figure 6.5(A), the maximum number of observed levels (filled circles) and the switching percentage (open circles) is plotted against the tunneling current at a sample bias of $10 \mathrm{mV}$ and a temperature of $77 \mathrm{~K}$. The switching 

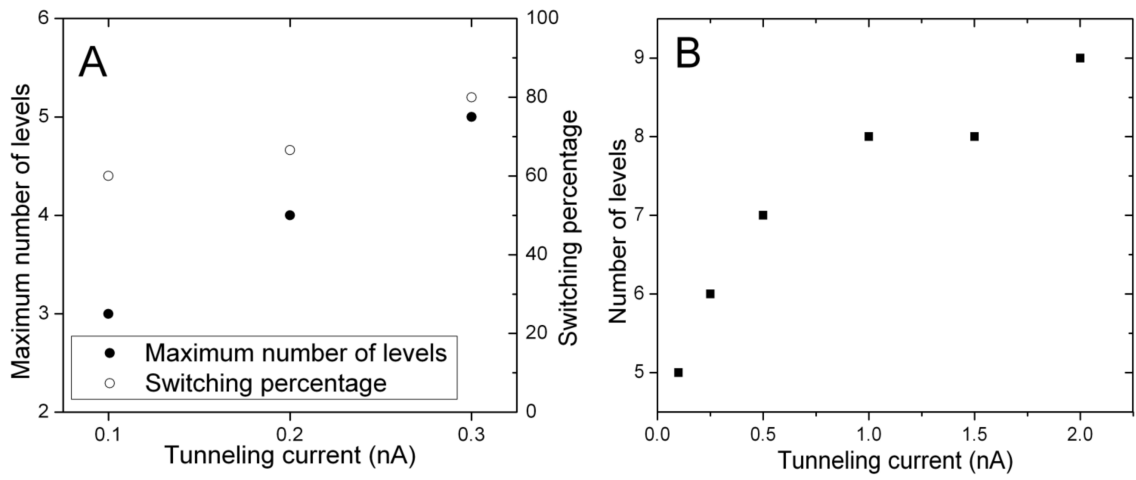

Figure 6.5: (A) Maximum number of observed levels versus tunneling current (filled circles) and switching percentage plotted against tunneling current (open circles). The sample bias was set to $10 \mathrm{mV}$ and the data were recorded at $77 \mathrm{~K}$. (B) Maximum number of observed levels versus tunneling current. The sample bias was set to $-0.5 \mathrm{~V}$ and the data were recorded at $77 \mathrm{~K}$.

percentage represents the percentage of traces where molecular switching has been observed. The increase in the switching percentage follows the higher switching rate at higher tunneling current. A clear trend, i.e. an increase in the number of levels with increasing current, is observed. We like to point out that even at a low sample bias of only $10 \mathrm{mV}$, electron induced dynamics are observed. The latter implies that the excitation threshold should lie below $10 \mathrm{mV}$. In Figure 6.5(B) the maximum number of levels is plotted against the tunneling current at a sample bias of $-0.5 \mathrm{~V}$. Also here an increase in the number of levels is observed with increasing tunneling current.

Based on our experimental observations we propose the following scenario for the observed dynamics of the $\beta$-CD molecules at such a lower voltage. A small fraction of the electrons tunnel inelastically and is able to excite vibronic, i.e. stretching, wagging, bending or rotational, modes of the molecule, provided that the energy of the electron $(\mathrm{eV})$ is larger than the threshold for excitation $(\hbar \omega)$, i.e. $\mathrm{eV}>\hbar \omega$. In a vibronic excited state the energy barrier to switch the molecule from configuration I to II (II to I) is reduced from its activation energy of $\mathrm{E}_{\text {act }(\mathrm{I} \rightarrow \mathrm{II})}\left(\mathrm{E}_{\text {act }(\mathrm{II} \rightarrow \mathrm{I})}\right)$. A Schematic energy diagram for such transitions is shown in Figure 6.6. With increasing current the probability to excite the $\beta$-CD molecule is enhanced and therefore it is likely that more excited levels will be observed in a given time interval. An increase of the sample bias also leads to enhanced dynamics for two reasons: (1) the 


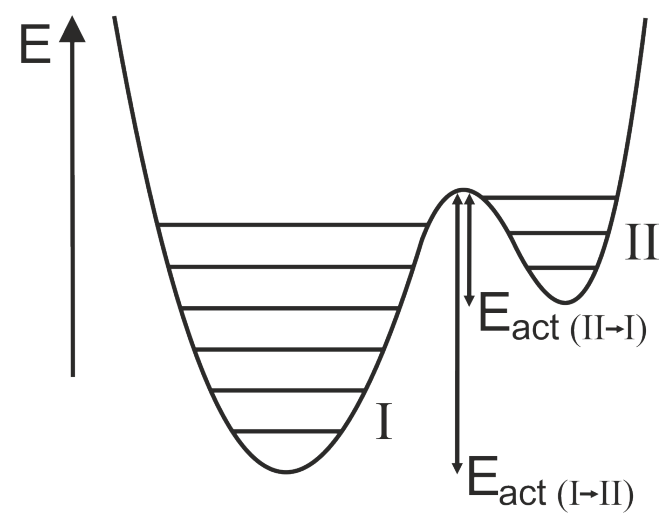

Figure 6.6: Schematic energy diagram of a molecule, which exhibits two conformational configurations (I and II). A transition from configuration I to II (II to I) will require an activation energy of $\mathrm{E}_{\operatorname{act}(\mathrm{I} \rightarrow \mathrm{II})}\left(\mathrm{E}_{\operatorname{act}(\mathrm{II} \rightarrow \mathrm{I})}\right)$. The transition between them can be facilitated by the excitation of vibronic modes of the molecules. The separation between adjacent excited vibronic states is $\hbar \omega$.

efficiency of excitation increases with increasing electron energy and (2) for sufficient high energies (voltage) higher lying excited states can be reached.

Since the vibronic modes of the intramolecular bonds of organic molecules are usually larger than $\approx 10-30 \mathrm{meV}$, we suggest that the excitation of the relatively heavy macrocycle of the $\beta$-CD molecule $(M=1135)$ induces the switching between the various conformational configurations of the molecule at low sample biases. Park et al.(151) found similar vibrational modes arising in $\mathrm{C}_{60}$ molecules on $\mathrm{Au}$ surface, as $\mathrm{C}_{60}$ is bound to the surface by van der Waals interactions. Since the mass of a $\beta$-CD macrocycle is almost double the mass of a $\mathrm{C}_{60}$ molecule, we expect that the vibrational quantum will be less than $5 \mathrm{meV} 1$. However, the actual value will depend on the exact number of the sulfur moieties attached to the Au surface.

\subsubsection{Current-Voltage Oscillations in $\beta$-Cyclodextrin Mono- layer}

The I-V curves recorded at room temperature on the $\beta$-CD SAMs exhibit small oscillations that seem to appear repeatedly above a certain bias voltage. Figure 6.7 shows an example of the differential conductance extracted from such an

\footnotetext{
${ }^{1}$ Harmonic oscillator frequency, $\omega=\sqrt{\frac{k}{m}}$, where $k$ is spring constant and $m$ is effective mass of the system.
} 
I-V curve plotted against bias voltage. The curve demonstrates that the peaks appear at regular voltage intervals which is a sign of resonance states in the tunnel junction. The peaks are numbered to identify the resonance states with onset occurring at bias value of $1.57 \mathrm{~V}$. A careful analysis reveals that these oscillations correspond to Gundlach oscillations (86, 87) in Fowler Nordheim tunneling regime.

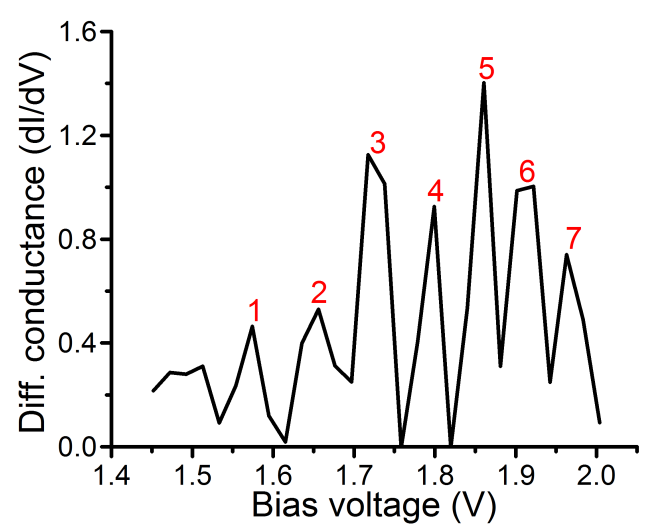

Figure 6.7: Differential conductance $\left(\frac{d I}{d V}\right)$ vs bias voltage shows oscillations in the range of $1.5 \mathrm{~V}$ to $2.0 \mathrm{~V}$. The peaks of the oscillations are numbered and correspond to the electronic states, $\mathrm{n}$.

Figure 6.8 shows energy corresponding to peak positions plotted against $\mathrm{n}^{2 / 3}$, where $\mathrm{n}$ is the peak number. It is well known from elementary quantum mechanics that for a rectangular potential well, energy of $n^{\text {th }}$ confined states is proportional to $\mathrm{n}^{2}$ while for a triangular potential well the energy of the quantum states is proportional to $\mathrm{n}^{2 / 3}$. An schematic is shown in the right side of Fig. 6.8. A linear fit on the left side of the figure confirms that the resonance states are characteristic of confined electronic states in a triangular potential well. This phenomena is referred as Gundlach oscillations (86, 87) where the transport is dominated by Fowler-Nordheim tunneling. Fowler-Nordheim tunneling occurs when the bias voltage exceeds the work function of substrate (or tip). As the sample bias crosses the work function of the substrate, the tunneling current exhibits oscillations owing to the fact that the field emitted electrons are reflected from the substrate leading to the formation of standing waves in a triangular potential well formed due to reshaping of the potential barrier. When the energy of the electrons is high enough to align with one of 
the energy levels of the triangular potential well, a higher transmission results in oscillations in the $\mathrm{I}-\mathrm{V}$ measurements.
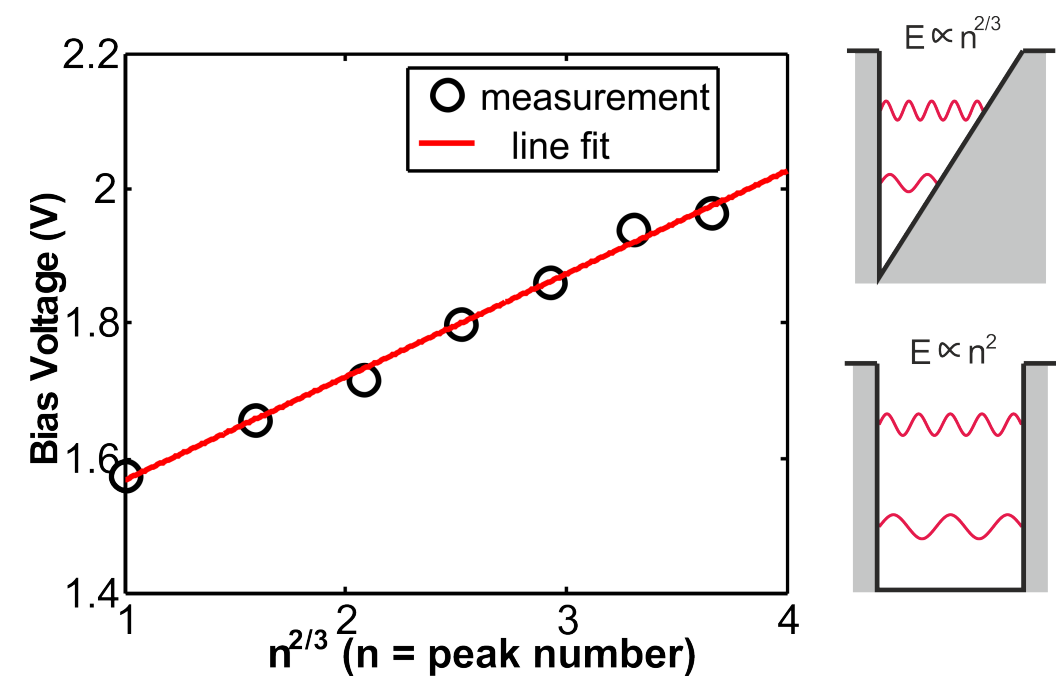

Figure 6.8: Left panel shows a plot of bias voltage against $n^{2 / 3}, n$ being the peak number. A linear fit indicates that the peaks correspond to electronic states of a triangular potential well. The right side shows two potential wells; triangular potential well with energy, $\mathrm{E} \propto \mathrm{n}^{2 / 3}$ (top) while for a rectangular potential well, energy, $\mathrm{E} \propto \mathrm{n}^{2}$ (bottom).

This means that the work function of the $\beta$-CD SAM/Au surface would be lower than $1.57 \mathrm{~V}$. A more accurate value of $1.41 \mathrm{~V}$, can be extracted from intercept of the line on the vertical-axis for $\mathrm{n}=0$ resonance state. The lowering of the work function of $\beta$-CD SAM/Au could be due to existence of dipole at the interface of $\mathrm{Au}$ and the monolayer. Transition voltage spectroscopy $(29,152,153)$ analysis also provides an indication that there exists a transition voltage of $\sim 1.2 \mathrm{~V}$ above which the transport is dominated by FowlerNordheim tunneling. However, because of the debatable nature of transition voltage spectroscopy (154 156), we can not make a firm conclusion on the barrier height and therefore on the work function. Another possibility could be the existence of a potential well on the surface of the $\beta$-CD SAM which is very similar to $\alpha, \omega$-mercaptoalkyl ferrocene/Au monolayer as reported by MüllerMeskamp et al.(19). Here, they associate Gundlach oscillations to the presence of a quantum well on the surface due to ferrocene moiety which is separated by vacuum barrier on one side and by alkyl legs on the other side.

For a total of ten curves the oscillation peaks were calculated. The first os- 
cillations always appear around bias voltage of $\sim 1.5 \mathrm{~V}$. Taking the exact value of the peaks for all ten curves into consideration, the average work function for the $\beta$-CD SAMs lies above a value of $\sim 1.41 \mathrm{~V}$. These observations were not reported in case of EGaIn macroscopic electrode junctions most probably due to the oscillations being averaged out in the large electrode-monolayer contact area.

\subsection{Conclusion}

We demonstrate that the transport measurements carried out on $\beta$-CD SAM on $\mathrm{Au}(111)$ using STM reveal various characteristics ascribed to the single molecule level. These characteristics are averaged out and hence not apparent when performing I-V measurements on macroscopic area junctions for example, using EGaIn technique. We find that the $\beta$-CD molecules exhibit a very rich dynamical behavior as reflected in the I-t spectroscopy which indicates different conformational states of the molecule. The dynamics is found to be temperature independent, but increase with increasing tunneling current and sample bias, revealing that the conformational changes of the $\beta$-CD molecules are induced by electrons that tunnel inelastically. Even for sample biases as low as $10 \mathrm{mV}$ we are able to induce transition between the various conformational configurations of the $\beta-\mathrm{CD}$ molecule. The I-V curves also show Gundlach oscillations which are characteristic of field emission resonance of electrons confined in a triangular potential well in the Fowler-Nordheim tunneling regime. This study corroborates the complexity of charge transport that occurs in molecular tunneling junctions. Therefore, a great care must be taken when presenting and interpreting the conclusions based on I-V data obtained from macroscopic molecular junctions. 


\section{Summary}

This thesis presents several experimental studies in molecular electronics using scanning tunneling microscopy (STM) and spectroscopy. Chapter 1 provides a brief introduction to molecular electronics and scanning tunneling microscopy. Chapter 2 covers a small description of STM setups used in the experiments presented in this thesis. Further, we have investigated electronic (transport) properties of a single octanethiol molecule, a single copper phthalocyanine $(\mathrm{CuPc})$ molecule and molecular assemblies of $\beta$-cyclodextrin $(\beta-\mathrm{CD})$ as presented in later chapters.

In chapter 3, we introduced Pt and Au modified Ge(001) surfaces as nanotemplates for immobilization of single molecules followed by investigations of nanocavities on a Pt/Ge(001) surface. Both Pt and Au modified Ge(001) surfaces provide well-ordered arrays of nanowires that are $1.6 \mathrm{~nm}$ apart. We observed that the $\mathrm{Pt} / \mathrm{Ge}(001)$ surface hosts a new phase consisting of densely packed and well-ordered arrays of nanocavities. The nanocavities are present on the $\beta$-terrace. The measured geometry of nanocavities is influenced by the local density of states as the nanocavities show up as ellipses at negative sample biases and as rectangles at positive sample biases. We witness an electronic shape transition of these cavities at low positive sample bias of 0.2 $\mathrm{V}$. The $\frac{d I}{d V}$ map exhibits a well-pronounced electronic state at $0.7 \mathrm{eV}$ in the region between neighboring nanocavities. The well-ordered structure between the nanocavities consists of symmetric and asymmetric tetramers rather than dimers.

In chapter 4, we investigated transport through a single octanethiol molecule trapped between an STM tip and a Pt/Ge(001) substrate. Current-distance (I-z) and current-voltage (I-V) spectroscopy were used to controllably trap the molecule between the tip and the substrate. From I-V spectroscopy, we extracted that as the electric field between the tip and the substrate exceeds 
the threshold value of 4-6 x $10^{9} \mathrm{Vm}^{-1}$, the molecules flips to bridge the gap between the substrate and the tip. This is termed as switched 'on' configuration as the current through the junction is substantially high. Reducing the field below this threshold value leads to the detachment of the octanethiol molecule and the current through the junction drops substantially. Therefore, this was termed as switch 'off' configuration. Using this scheme, we performed I-z spectroscopy at various temperatures to ascertain the transport mechanism of this junction. This set of I-z spectroscopy revealed that the conductance of the junction is temperature independent, demonstrating that the main transport mechanism is dominated by either direct quantum-mechanical tunneling or ballistic transport.

In chapter 5, we demonstrated that we are able to structurally and electronically decouple the $\mathrm{Cu}^{2+}$ core of a $\mathrm{CuPc}$ molecule from the underlying $\mathrm{Au} / \mathrm{Ge}(001)$ substrate. We achieved this by trapping the molecule in a molecular bridge configuration between two adjacent $\mathrm{Au}$-induced $\mathrm{Ge}(001)$ nanowires. As each of the two lobes of a $\mathrm{CuPc}$ molecule bind to the adjacent nanowires the core stays in the middle of the trough which has a depth of at least $5 \AA$. The core of the $\mathrm{CuPc}$ molecule stays invisible in empty-states STM images up to a sample bias of $3.5 \mathrm{~V}$. However, charging of the core occurs at sample biases larger than $3.5 \mathrm{~V}$ as the core becomes bright in STM images recorded at $5.0 \mathrm{~V}$. CuPc molecules diffuses on the surface which is induced by tunneling electrons. The molecules with speckles on the lobes exhibit two level dynamics as revealed in the current-time (I-t) spectroscopy.

In chapter 6 , we study the transport measurements carried out on a $\beta$-CD self-assembled monolayer (SAM) on $\mathrm{Au}(111)$ surface using I-V and I-t spectroscopy. The STM measurements reveal various transport properties ascribed to the single molecule level which are averaged out and hence not apparent when performing $\mathrm{I}-\mathrm{V}$ measurements on macroscopic area junctions using the EGaIn technique. $\beta$-CD molecules exhibit a very rich dynamical behavior as reflected in the I-t spectroscopy which indicates different conformational states of the molecule. We performed I-t spectroscopy at room temperature and at $77 \mathrm{~K}$ and found that the dynamics is temperature independent. I-t traces recorded at various sample biases and setpoint currents showed that the dynamics increases with increasing tunneling current and sample bias. This means that the conformational changes of the $\beta$-CD molecules are induced by electrons that tunnel inelastically. The combination of I-t and I-V 
spectroscopy studies showed that great care must be taken while presenting and interpreting the transport measurements data obtained from macroscopic molecular junctions. 


\section{Samenvatting}

In dit proefschrift worden verschillende experimentele onderzoeken in moleculaire elektronica gepresenteerd. Er wordt gebruik gemaakt van scanning tunneling microscopie (STM) en spectroscopie. In Hoofdstuk 1 wordt een beknopte introductie gegeven wat betreft moleculaire elektronica en scanning tunneling microscopie. Vervolgens worden in Hoofdstuk 2 de verschillende STM opstellingen omschreven waarmee de experimenten zijn uitgevoerd. Verder hebben we de elektron (transport) eigenschappen onderzocht van een enkel octaanthiol molecuul, een enkel koper phtalocyanine $(\mathrm{CuPc})$ molecuul en $\beta$-cyclodextrin ( $\beta$-CD) moleculen, zoals zal worden besproken in latere hoofdstukken.

In hoofdstuk 3 introduceren we Pt en Au gemodificeerde Ge(001) oppervlakken als nanotemplates voor de immobilisatie van enkele moleculen. Dit wordt gevolgd door onderzoek aan nanoholtes op een Pt/Ge(001) oppervlak. De Pt en ook de $\mathrm{Au}$ gemodificeerde Ge(001) oppervlakken bestaan uit netjes gerangschikte rijen van nanodraden die op een onderlinge afstand van 1.6 $\mathrm{nm}$ liggen. We hebben ontdekt dat het $\mathrm{Pt} / \mathrm{Ge}(001)$ oppervlak de gastheer is voor een nieuwe fase, bestaande uit dicht op elkaar gepakte en ordelijk gerangschikte netwerk van nanoholtes. De nanoholtes worden alleen gevonden op $\beta$-terrassen. De gemeten geometrie van de nanoholtes wordt beïnvloed door de lokale toestandsdichtheid aangezien de nanoholtes ellipsvormig zijn bij negatieve substraatspanningen en rechthoekig bij positieve substraatspanningen. De elektronische vormverandering van deze holtes vindt plaats bij een lage positieve substraatspanning van $0.2 \mathrm{~V}$. De $2 \mathrm{D}$ representatie van de differentiële geleidbaarheid laat een duidelijke elektronische toestand zien in het gebied tussen aangrenzende nanoholtes bij $0.7 \mathrm{eV}$. De sterk geordende structuur tussen de nanoholtes blijkt uit symmetrische en asymmetrische tetrameren te bestaan. 
In hoofdstuk 4 onderzoeken we transport door een enkel octaanthiol molecuul dat gevangen is tussen een STM tip en een Pt/Ge(001) substraat. Stroomafstand (I-z) en stroom-spanning (I-V) spectroscopie zijn gebruikt om op een controleerbare wijze het molecuul te vangen tussen de tip en het substraat. Uit I-V spectroscopie blijkt dat wanneer het elektrisch veld sterker wordt dan de drempelwaarde van 4-6 × $10^{9} \mathrm{Vm}^{-1}$, het molecuul gevangen wordt tussen tip en oppervlak. Wij hebben deze configuratie als "aan" aangeduid, aangezien de stroom door deze schakeling hoger wordt. Wanneer we nu het elektrisch veld verminderen tot onder deze drempelwaarde zien we dat dit leidt tot de ontkoppeling van het octaanthiol molecuul en de tunnelstroom wordt aanzienlijk lager. Daarom hebben wij dit de "uit" configuratie genoemd. Ook hebben wij I-z spectroscopie uitgevoerd, met het molecuul gebonden aan de tip, bij verschillende temperaturen om het transportmechanisme van deze junctie vast te stellen. Deze serie van I-z spectroscopie metingen laat zien dat de geleiding van deze junctie temperatuur onafhankelijk is. Hieruit blijkt dat het transport mechanisme wordt gedomineerd door coherente kwantummechanische tunneling of door ballistische transport.

In hoofdstuk 5 demonstreren we dat wij in staat zijn om op een structurele en ook elektronische wijze de $\mathrm{Cu}^{2+}$ kern van een $\mathrm{CuPc}$ molecuul te ontkoppelen van het onderliggende $\mathrm{Au} / \mathrm{Ge}(001)$ substraat. We hebben dit bereikt door het molecuul in een brugconfiguratie tussen twee aaneengrenzende $\mathrm{Au}$ geïnduceerde nanodraden te vangen. Omdat alle vier de lobben van het $\mathrm{CuPc}$ molecuul gebonden zijn aan de nanodraden, blijft de $\mathrm{Cu}^{2+}$ kern gepositioneerd in het midden tussen de nanodraden boven een tenminste $5 \AA$ diepe geul. De kern van het $\mathrm{CuPc}$ molecuul blijft onzichtbaar bij lege-toestand STM afbeeldingen tot een substraatspanning van $3.5 \mathrm{~V}$. Echter, het opladen van de kern geschiedt bij een substraatspanning hoger dan $3.5 \mathrm{~V}$ aangezien de kern helder oplicht in STM metingen opgenomen bij 5.0 V. De diffusie van $\mathrm{CuPc}$ moleculen over het oppervlak wordt geïnduceerd door tunnelende elektronen. De moleculen met de gespikkeld uitziende lobben vertonen dynamica zoals blijkt uit de stroom-tijd (I-t) spectroscopie.

In hoofdstuk 6 bestuderen we de transportmetingen uitgevoerd op een $\beta$-CD SAM op een $\mathrm{Au}(111)$ oppervlak, gebruik makend van I-V en I-t spectroscopie. De STM brengt verschillende transport eigenschappen aan het licht die kunnen worden toegeschreven aan het gedrag van een enkel molecuul. Deze eigenschappen worden uitgemiddeld en zijn dus niet zichtbaar wanneer I-V 
metingen worden uitgevoerd op een macroscopisch gebied, zoals bijvoorbeeld voor de EGaIn techniek. $\beta$-CD moleculen vertonen een zeer rijk dynamisch gedrag, zoals blijkt uit de I-t spectroscopie. Dit toont aan dat het molecuul zich in verschillende geometrische configuraties kan bevinden. Uit I-t metingen uitgevoerd bij kamertemperatuur en bij $77 \mathrm{~K}$ blijkt dat de dynamica van de moleculen temperatuur onafhankelijk is. Ook is duidelijk dat er meer dynamica plaatsvindt wanneer wordt gemeten bij een hogere tunnel stroom of substraatspanning. Dit betekent dat veranderingen in de geometrische configuratie van het $\beta$-CD molecuul worden geïnduceerd door inelastisch tunnelende elektronen. De combinatie van I-t en I-V spectroscopie metingen laat zien dat grote voorzichtigheid in acht genomen moet worden bij het presenteren en interpreteren van data komend van transport metingen op macroscopische moleculaire verbindingen. 


\section{References}

[1] A. Aviram and M. A. Ratner, Chem. Phys. Lett. 29, 277 (1974). 2,32

[2] M. Fuechsle et al., Nature N 7, 242 (2012). 2

[3] K. Likharev, Electronics below 10 nm, Amsterdam: Elsevier, 2003. 2,32

[4] B. Xu and N. J. Tao, Science 301, 1221 (2003). 2, 4, 32

[5] S. Karthäuser, J. Phys.: Condens. Matter 23, 013001 (2011). 2, $5,6,32,33$. 38, 43,60

[6] G. Nazin, X. Qiu, and W. Ho, Science 302, 77 (2003). 2, 5,47

[7] J. J. Parks et al., Science 328, 1370 (2010). 3

[8] N. J. Tao, Nature Nanotech. 1, 173 (2006). 3

[9] J. M. van Ruitenbeek et al., Rev. Sci. Instrum. 67, 108 (1996). 232

[10] J. Reichert et al., Phys. Rev. Lett. 88, 176804 (2002). 2, 32, 34, 60, 62

[11] H. Park, A. K. L. Lim, A. P. Alivisatos, J. Park, and P. L. McEuen, Appl. Phys. Lett. 75, 301 (1999). 2

[12] C. A. Martin, D. Ding, H. S. J. van der Zant, and J. M. van Ruitenbeek, New Journal of Physics 10, 065008 (2008). 3

[13] J. G. Kushmerick et al., Phys. Rev. Lett. 89, 086802 (2002). 3,32

[14] D. J. Wold and C. D. Frisbie, J. Am. Chem. Soc. 123, 5549 (2001). 34

[15] R. Chiechi, E. Weiss, M. Dickey, and G. Whitesides, Angew. Chem. Int. Ed. 47, 142 (2008). 4 60 
[16] Y. Selzer, A. Salomon, and D. Cahen, J. Phys. Chem. B 106, 10432 (2002). 4

[17] K. Wimbush et al., Angew. Chem. Int. Ed. 49, 10176 (2010). 4 60

[18] S. Y. Quek et al., Nature Nanotechnology 4, 230 (2009). 4 32

[19] L. Müller-Meskamp et al., Small 5, 496 (2009). 4, 71

[20] F. Chen, J. Hihath, Z. Huang, X. Li, and N. Tao, Annu. Rev. Chem. 58, 535 (2007). 4.32

[21] D. Kockmann, B. Poelsema, and H. J. W. Zandvliet, Nano Lett. 9, 1147 (2009). 4, 21, 32, 35, 39, 60,

[22] E. Leary et al., Nano Lett. 11, 2236 (2011). 3260

[23] L. Lafferentz et al., Science 323, 1193 (2009). 4] 32

[24] G. Schull, T. Frederiksen, A. Arnau, D. Sánchez-Portal, and R. Berndt, Nature Nanotech. 6, 23 (20111). 5

[25] R. Temirov, A. Lassise, F. B. Anders, and F. S. Tautz, Nanotechnology 19, 065401 (2008). 5, 32,60

[26] J. Schaffert et al., Nature Materials 12, 223 (2013). $5,55,56$

[27] S. Jan Van Der Molen and P. Liljeroth, Journal of Physics Condensed Matter 22, 133001 (30pp) (2010). 5

[28] H. Song, M. A. Reed, and T. Lee, Adv. Mater. 23, 1583 (2011). 56

[29] J. M. Beebe, B. Kim, J. Gadzuk, D. Frisbie, and J. G. Kushmerick, Phys. Rev. Lett. 97, 026801 (2006). 5,71

[30] G. Binnig, H. Rohrer, C. Gerber, and E. Weibel, Phys. Rev. Lett. 49, 57 (1982). 6

[31] J. A. Stroscio and D. M. Eigler, Science 254, 1319 (1991). 6

[32] C. Chen, Introduction to Scanning Tunneling Microscopy, New York: Oxford University Press, 1993. 6, 8, 9

[33] C. Toumey, Nature Nanotech. 7, 205 (2012). 6 
[34] C. Weiss, C. Wagner, R. Temirov, and F. S. Tautz, J. Am. Chem. Soc. 132, 11864 (2010). 6

[35] H. J. Lee and W. Ho, Science 286, 1719 (1999). 6

[36] Y. Jiang, Q. Huan, L. Fabris, G. C. Bazan, and W. Ho, Nature Chem. 5, 36 (2013). 6

[37] A. Saedi, A. van Houselt, R. van Gastel, B. Poelsema, and H. J. W. Zandvliet, Nano Lett. 9, 1733 (2009). 7, 22, 61

[38] L. Gao et al., Phys. Rev. L 101, 197209 (2008). 7

[39] J. G. Simmons, Journal of Applied Physics 34, 1793 (1963). 8, 38

[40] H. LIN et al., Front. Phys. China 5, 369 (2010). 9

[41] J. Tersoff and D. R. Hamann, Phys. Rev. B 31, 805 (1985). 9

[42] C. J. Chen, Phys. Rev. Lett. 65, 448 (1990). 9

[43] H. Zandvliet and A. Van Houselt, Annual Review of Analytical Chemistry 2, 37 (2009). 10,61

[44] S. Berner et al., Angewandte Chemie 46, 5115 (2007). 20

[45] N. R. Shiju and V. V. Guliants, Appl. Catal. A Gen. 356, 1 (2009). 20

[46] J. V. Barth, G. Costantini, and K. Kern, Nature 437, 671 (2005). 20

[47] J. Otsuki, Coord. Chem. Rev. 254, 2311 (2010). 20

[48] H. Spillmann et al., Adv. Mater. 18, 275 (2006).

[49] M. Fischer, A. van Houselt, D. Kockmann, B. Poelsema, and H. J. W. Zandvliet, Phys. Rev. B 76, 1 (2007). 20, 21, 22, 24, 25, 28, 34

[50] A. Dmitriev, N. Lin, J. Weckesser, J. V. Barth, and K. Kern, J. Phys. Chem. B 106, 6907 (2002).

[51] N. Lin et al., Angew. Chem. Int. Ed. 44, 1488 (2005). 20

[52] M. Jurow, A. E. Schuckman, J. D. Batteas, and C. M. Drain, Coord. Chem. Rev. 254, 2297 (2010). 20

[53] H. Liang et al., Coord. Chem. Rev. 253, 2959 (2009). 20 
[54] S. van Dijken, L. C. Jorritsma, and B. Poelsema, Phys. Rev. B 61, 14047 (2000). 20

[55] P. C. dos Santos Claro et al., ACS Nano 2, 2531 (2008). 20

[56] S. van Dijken, D. de Bruin, and B. Poelsema, Phys. Rev. Lett. 86, 4608 (2001). 20

[57] O. Gurlu, O. A. O. Adam, H. J. W. Zandvliet, and B. Poelsema, Appl. Phys. Lett. 83, 4610 (2003). 20, 21, 25

[58] J. Wang, M. Li, and E. I. Altman, Phys. Rev. B 70, 233312 (2004).

[59] A. van Houselt, M. Fischer, B. Poelsema, and H. J. W. Zandvliet, Phys. Rev. B 78, 233410 (2008). 20, 22, 23, 24, 47, 49, 51, 52

[60] A. Saedi, R. P. Berkelaar, A. Kumar, B. Poelsema, and H. J. W. Zandvliet, Phys. Rev. B 82, 165306 (2010). 21

[61] N. Oncel et al., Phys. Rev. Lett. 95, 116801 (2005). 22, 29,34

[62] A. van Houselt et al., Surf. Sci. 602, 1731 (2008). 22

[63] D. Kockmann, T. Mocking, A. Van Houselt, B. Poelsema, and H. Zandvliet, J. Phys. Chem. C 113, 17156 (2009). 22, 23, 24, 51

[64] J. Wang, M. Li, and E. Altman, Phys. Rev. B 70, 233312 (2004). 22,24

[65] J. Schäfer, C. Blumenstein, S. Meyer, M. Wisniewski, and R. Claessen, Phys. Rev. Lett. 101, 236802 (2008). $22,24,51$

[66] A. van Houselt, D. Kockmann, T. F. Mocking, B. Poelsema, and H. J. W. Zandvliet, Phys. Rev. Lett. 103, 209701 (2009). 24,51

[67] J. Schäfer et al., New J. Phys. 11, 125011 (2009). 47

[68] K. Nakatsuji et al., Phys. Rev. B 80, 081406 (2009). 47

[69] R. Niikura, K. Nakatsuji, and F. Komori, Phys. Rev. B 83, 035311 (2011). 53

[70] K. Nakatsuji and F. Komori, Nature Physics 8, 174 (2012).

[71] C. Blumenstein et al., Nature Physics 7, 776 (2011). 24

[72] C. Blumenstein et al., Nature Physics 8, 174 (2012). 
[73] R. Heimbuch, M. Kuzmin, and H. J. W. Zandvliet, Nature Physics 8, 697 (2012). 22,51

[74] C. Blumenstein et al., Phys. Rev. Lett. 107, 165702 (2011). 24

[75] R. Heimbuch, M. Kuzmin, and H. Zandvliet, Nature Physics 8, 697 (2012). 24

[76] R. P. Berkelaar et al., Journal of Physical Chemistry C 115, 2268 (2011). 24. 47, 48, 49, 52, 54

[77] H. J. W. Zandvliet, B. S. Swartzentruber, W. Wulfhekel, B. J. Hattink, and B. Poelsema, Phys. Rev. B 57, 6803 (1998). 25, 48

[78] H. J. W. Zandvliet, Physics Reports 388, 1 (2003). 25, 48

[79] H. G. Zhang et al., J. Phys.: Condens. Matter 22, 302001 (2010). 28

[80] F. Klappenberger et al., Nano Lett. 9, 3509 (2009). 28

[81] H. Oughaddou et al., Phys. Rev. B 61, 5692 (2000). 29

[82] K. W. Hipps, Science 294, 536 (2001). 32

[83] W. Haiss et al., J. Am. Chem. Soc. 125, 15294 (2003). 32,60

[84] J. S. Meisner et al., Nano Lett. 11, 1575 (2011). 32

[85] C. Toher et al., Phys. Rev. B 83, 155402 (2011). 32

[86] K. Gundlach, Solid-State Electronics 9, 949 (1966). 33,70

[87] C. L. Lin et al., Phys. Rev. Lett. 99, 216103 (2007). 33,70

[88] G. Sedghi et al., Nature Nanotechnology 6, 517?523 (2011). 33

[89] R. H. M. Smit et al., Nature 419, 906 (2002). 34

[90] J. M. van Ruitenbeek et al., Review of Scientific Instruments 67, 108 (1996). 34

[91] O. Gurlu, O. A. Adam, H. J. W. Zandvliet, and B. Poelsema, Appl. Phys. Lett. 83, 4610 (2003). 34

[92] H. B. Akkerman and B. de Boer, J. Phys.: Condens. Matter 20, 013001 (2008). $34,36,43$ 
[93] P. A. van Hal et al., Nature Nanotechnology 3, 749 (2008).

[94] W. Wang, T. Lee, and M. A. Reed, Phys. Rev. B 68, 035416 (2003). 36

[95] J. K. Tomfohr and O. F. Sankey, Phys. Rev. B 65, 245105 (2002). 37

[96] J. G. Simmons, Journal of Applied Physics 34, 2581 (1963). 38

[97] A. van Houselt and H. J. W. Zandvliet, Reviews of Modern Physics 82, 1593 (2010). 3961

[98] P. K. Schelling and P. Keblinski, Phys. Rev. B 68, 035425 (2003). 43

[99] S. J. V. D. Molen and P. Liljeroth, J. Phys.: Condens. Matter 22, 133001 $(2010) .4360$

[100] W. Wang, T. Lee, and M. A. Reed, Proceedings of the IEEE 93, 1815 (2005). 43,44

[101] P. Peumans, A. Yakimov, and S. R. Forrest, J. appl. Phys. 93, 3693 (2003). 46

[102] R. Lunt, J. Benziger, and S. Forrest, Adv. Mater. 19, 4229 (2007). 46

[103] J. Blochwitz, M. Pfeiffer, T. Fritz, and K. Leo, Appl. Phys. Lett. 73, 729 (1998). 46

[104] C. Isvoranu et al., J. Chem. Phys. 131, 214709 (2009). 46

[105] Z. Shi and N. Lin, J. Am. Chem. Soc. 131, 5376 (2009). 46

[106] C. Isvoranu et al., J. Chem. Phys. 134, 114711 (2011). 46

[107] C. Isvoranu et al., J. Chem. Phys. 134, 114710 (2011).

[108] Q. Li, J. Yang, Z. Li, J. Hou, and Q. Zhu, Acta Phys. Sin. 50, 1877 (2001). 46

[109] X. Qiu, G. Nazin, and W. Ho, Phys. Rev. Lett. 92, 206102 (2004). 46, 50,54

[110] S. Wu, G. Nazin, X. Chen, X. Qiu, and W. Ho, Phys. Rev. Lett. 93 (2004). 46. 50.54

[111] A. Schaffer, M. Gouterman, and E. Davidson, Theoretica Chimica Acta 30, 9 (1973). 46, 50

[112] J. Ren et al., J. Chem. Phys. 134, 194706 (2011). 46 
[113] D. Zahn, G. Gavrila, and M. Gorgoi, Chemical Physics 325, 99 (2006). 50

[114] J. Schäfer, C. Blumenstein, S. Meyer, M. Wisniewski, and R. Claessen, Phys. Rev. Lett. 103, 209702 (2009). 51

[115] X. Lu, K. Hipps, X. Wang, and U. Mazur, Journal of the American Chemical Society 118, 7197 (1996). 52

[116] D. Ferry and S. Goodnick, Transport in nanostructures, Cambridge University Press, 1997. 54

[117] X. Qiu, G. Nazin, and W. Ho, Phys. Rev. Lett. 93, 196806 (2004). 54

[118] A. Zhao et al., Science 309, 1542 (2005). 54

[119] J. K. Gimzewski et al., Science 281, 531 (1998). 54

[120] B. C. Stipe, M. A. Rezaei, and W. Ho, Science 279, 1907 (1998). 54

[121] N. Henningsen et al., J. Phys. Chem. C 111, 14843 (2007). 54

[122] C. Joachim, J. Gimzewski, and A. Aviram, Nature 408, 541 (2000). 60

[123] R. L. McCreery and A. J. Bergren, Adv. Mater. 21, 4303 (2009). 60

[124] C. Nijhuis, W. Reus, J. Barber, M. Dickey, and G. Whitesides, Nano Lett. 10, 3611 (2010). 60

[125] X. Li et al., Journal of the American Chemical Society 129, 11535 (2007).

[126] Y. Selzer, M. Cabassi, T. Mayer, and D. Allara, Journal of the American Chemical Society 126, 4052 (2004).

[127] H. Akkerman, P. Blom, D. De Leeuw, and B. De Boer, Nature 441, 69 (2006). 60

[128] R. Haag, M. Rampi, R. Holmlin, and G. Whitesides, Journal of the American Chemical Society 121, 7895 (1999).

[129] T.-W. Kim, G. Wang, H. Lee, and T. Lee, Nanotechnology 18, 315204 (2007).

[130] C. Richter, D. Stewart, D. Ohlberg, and R. Williams, Applied Physics A: Materials Science and Processing 80, 1355 (2005). 60

[131] L. Lafferentz et al., Science 323, 1193 (2009). 60 
[132] S.-Y. Jang, P. Reddy, A. Majumdar, and R. Segalman, Nano Lett. 6, 2362 (2006). 60

[133] M. Beulen et al., Chemistry - A European Journal 6, 1176 (2000). 60, 63, 64

[134] J. Huskens, M. Deij, and D. Reinhoudt, Angew. Chem. Int. Ed. 41, 4467 (2002). 61

[135] C. Bruinink et al., Chemistry - A European Journal 11, 3988 (2005). 60

[136] C. Nijhuis, J. Huskens, and D. Reinhoudt, Journal of the American Chemical Society 126, 12266 (2004). 60

[137] C. A. Nijhuis, Y. Fang, W. Knoll, J. Huskens, and D. N. Reinhoudt, Langmuir 21, 7866 (2005). 60, 61

[138] C. Nijhuis, K. Dolatowska, B. Ravoo, J. Huskens, and D. Reinhoudt, Chemistry - A European Journal 13, 69 (2007). 60, 61

[139] C. Nijhuis, B. Boukamp, B. Ravoo, J. Huskens, and D. Reinhoudt, Journal of Physical Chemistry C 111, 9799 (2007). 60

[140] C. Nijhuis et al., Langmuir 22, 9770 (2006). 61

[141] A. Moore et al., Journal of the American Chemical Society 129, 10352 (2007). 61

[142] A. Jewell et al., J. Phys.: Condens. Matter 22, 264006 (2010). 61

[143] Q. Liu et al., Phys. Rev. Lett. 104, 166104 (2010). 61

[144] A. Van Houselt, R. Van Gastel, B. Poelsema, and H. Zandvliet, Phys. Rev. Lett. 97, 266104 (2006). 61

[145] T. Mocking, D. Stam, B. Poelsema, and H. Zandvliet, Surf. Sci. 604, 2021 (2010).

[146] A. Van Houselt, B. Poelsema, and H. Zandvliet, J. Phys.: Condens. Matter 22, 264004 (2010). 61

[147] N. Battaglini et al., Ultramicroscopy 107, 958 (2007). 61

[148] M. Beulen et al., Langmuir 14, 6424 (1998). 62, 63, 64,65 
[149] L. Patrone, J. Soullier, and P. Martin, Physica Status Solidi (B) 247, 1867 (2010). 66

[150] B.-Y. Choi et al., Phys. Rev. Lett. 96, 156106 (2006). 67

[151] H. Park et al., Nature 407, 57 (2000). 69

[152] G. Ricoeur, S. Lenfant, D. Guérin, and D. Vuillaume, J. Phys. Chem. C 116, $20722(2012) .71$

[153] J. M. Beebe, B. Kim, D. Frisbie, and J. G. Kushmerick, ACS Nano 2, 827 (2008). 71

[154] E. H. Huisman, C. M. Guédon, B. J. van Wees, and S. J. van der Molen, Nano Lett. 9, 3909 (2009). 71

[155] M. L. Trouwborst et al., Nano Lett. 11, 614 (2011).

[156] A. Vilan, D. Cahen, and E. Kraisler, ACS Nano 7, 695 (2013). 71 


\section{Publication List}

[1] R.P. Berkelaar, H. Sode, T.F. Mocking, A. Kumar, B. Poelsema, and H.J.W. Zandvliet. Molecular bridges. Journal of Physical Chemistry C, 115(5):2268, 2011.

[2] R. Heimbuch, H. Wu, A. Kumar, B. Poelsema, G.J. Vancso, P.M. Schön, and H.J.W. Zandvliet. Variable-temperature study of the transport through a single octanethiol molecule. Physical Review B, 86:075456, 2012.

[3] A. Kumar, R. Heimbuch, B. Poelsema, and H.J.W. Zandvliet. Controlled transport through a single molecule. Journal of Physics: Condensed Matter, 24:082201, 2012.

[4] A. Kumar, R. Heimbuch, K.S. Wimbush, H. Atesci, A. Acun, D.N. Reinhoudt, A.H. Velders, and H.J.W. Zandvliet. Electron induced dynamics of hepthathioether $\beta$-cyclodextrin molecules. Small, 8:317, 2012.

[5] A. Kumar, S. Kumar, J.M. Boter, C. Hellenthal, J.E. ten Elshof, and H.J.W. Zandvliet. Imaging of $\mathrm{Ti}_{0.87} \mathrm{O}_{2}$ nanosheets using scanning tunneling spectroscopy. Applied Surface Science, 265:201, 2013.

[6] A. Kumar, B. Poelsema, and H.J.W. Zandvliet. Self-organized nanocavity arrays on Pt/Ge(001). Journal of Physical Chemistry C, 115(14):67266729, 2011.

[7] M. Kuzmin, A. Kumar, B. Poelsema, and H.J.W. Zandvliet. Decoupling of copper core in a single copperphthalocyanine molecule. Journal of Chemical Physics, 138:114302, 2013. 
[8] T.F. Mocking, A. Kumar, and R.P. Berkelaar. Moleculaire bruggen, dynamica en massatransport op nanoschaal. NEVACBLAD, 49:7, 2011.

[9] A. Saedi, R.P. Berkelaar, A. Kumar, B. Poelsema, and H.J.W. Zandvliet. Adsorption of cu phthalocyanine molecules on Pt modified Ge(001): a scanning tunnelling microscopy study. Physical Review B, 82:165306, 2010.

[10] K. Sotthewes, H. Wu, A. Kumar, G.J. Vancso, P.M. Schön, and H.J.W. Zandvliet. Molecular dynamics and energy landscape of decanethiolates in self-assembled monolayers on $\mathrm{Au}(111)$ by STM. Langmuir, 29:3662, 2013.

[11] H. Wu, K. Sotthewes, A. Kumar, G.J. Vancso, P.M. Schön, and H.J.W. Zandvliet. Dynamics of decanethiol self-assembled monolayers on $\mathrm{Au}(111)$ studied by scanning tunnelling microscopy. Langmuir, 29:2250, 2013. 


\section{Acknowledgements}

During my PhD years, I had several opportunities to meet many wonderful people. My PhD would not be easy and this thesis would not come to this shape without their presence around me. I would like to take this opportunity to thank them all for their support, cooperation, comfort, and wishes, which added colors in my life.

Firstly, I want to express my gratitude to my supervisor, Prof. Harold Zandvliet for giving me an opportunity to work in his group and supervising me throughout the project. In addition to the scientific knowledge, his infectious and ceaseless energy, friendly and caring nature has been very inspiring and encouraging all the time.

I thank Bene for his invaluable comments during preparation of several manuscripts of the papers and his insights during the work discussions.

I like to thank Prof. Briels, Prof. de Leeuw, Prof. Köhler, Prof. Huskens, Prof. Brinkman and Dr. van Houselt for being in my graduation committee and for taking the time to read the manuscript.

My sincere thanks go Hans and Herman for their indispensable technical support I needed on various occasions. I like to thank former secretary, Rianne, and the present secretary of the group, Simone, for the administrative helps.

I also thank Herbert, Gregor, Stefan, and Raoul for the useful suggestions and remarks they provided during work discussions, and for the nice conversations we had during lunch hours and coffee breaks.

My thanks to René for being a great master student and a good colleague later on. Also, thanks for organizing the skiing trip to Bottrop and Oktoberfest. I wish you all the best.

I also had opportunities to collaborate with Suresh and Kim Wimbush 
whom I want to thank and wish them all the best. Also, thanks to Mikhail for the work we did together on CuPc. It was a great learning experience working with you.

I am also thankful to former group members Amir, Frank, Waqar, Arzu for providing a comfortable and welcoming stay in the group at the beginning. All the best to you guys.

Thanks to Maciek for being a great friend all these years. I never learned history better and saw it so closely than during the Poland trip we made together with Lucky. It was always fun being with you. Your inquisitiveness towards science, history, and technology is very inspiring. I wish you all the best.

I admire composure and discipline of Tijs and in subtle way he inspires me a lot. Thanks for being a great colleague and officemate. The US trip was fun; I couldn't learn better the nuances of hiking. I wish you all the best.

Thanks to Daniel and Vasilisa for the pleasant conversations during lunch and coffee breaks and also for inviting me to play poker, game of thrones etc. I wish you all the best.

It was very pleasant working with Kai and Hairong on self-assembled monolayers using RHK STM. Hairong, the strawberries from your garden and the Chinese food you prepared were delicious. Thanks and wish you guys all the best.

I also like to thank Ali, Imtiaz, Patrick, Robin, Eric, and Chris for all the nice moments in the group and during other occasions.

I am grateful to my friends Pankaj, Vivien, Ashvin, Aga, Deepak for being great friends and bringing joy to my life on so many occasions. Wish you lots of success.

Finally, I would like to thank my parents and relatives for their love and support during all these years. 


ISBN: 978-90-365-0527-7 